\title{
Electronic, Structural and Functional Versatility in Tetrathiafulvalene-Lanthanide Metal-Organic Frameworks
}

Javier Castella-Gil, Samuel Mañas-Valero, Iñigo J. Vitórica-Yrezábal, Duarte Ananias, João Rocha, Raul Santiago, Stefan T. Bromley, José J. Baldoví, Eugenio Coronado, Manuel Souto, Guillermo Minguez Espallargas

Submitted date: 24/07/2019 - Posted date: 26/07/2019

Licence: CC BY-NC-ND 4.0

Citation information: Castella-Gil, Javier; Mañas-Valero, Samuel; Vitórica-Yrezábal, Iñigo J.; Ananias, Duarte; Rocha, João; Santiago, Raul; et al. (2019): Electronic, Structural and Functional Versatility in Tetrathiafulvalene-Lanthanide Metal-Organic Frameworks. ChemRxiv. Preprint.

Tetrathiafulvalene-Lanthanide (TTF-Ln) Metal-Organic Frameworks (MOFs) are an interesting class of multifunctional materials in which porosity can be combined with electronic properties such as electrical conductivity, redox activity, luminescence and magnetism. Herein we report a new family of isostructural TTF-Ln MOFs, denoted as MUV-5(Ln) ( $L n=G d, T b, D y, H o, ~ E r)$, exhibiting semiconducting properties as a consequence of the short intermolecular $S$...S contacts established along the chain direction between partially oxidised TTF moieties. In addition, this family shows photoluminescence properties and single-molecule magnetic behaviour, finding near-infrared (NIR) photoluminescence in the $\mathrm{Yb} / \mathrm{Er}$ derivative and slow relaxation of the magnetisation in the Dy and Er derivatives. As such properties are dependent on the electronic structure of the lanthanide ion, we emphasise the immense structural, electronic and functional versatility of this class of materials.

File list (2)

Souto_Minguez_MUV-5_Manuscript.pdf (1.01 MiB) view on ChemRxiv - download file 


\title{
Electronic, Structural and Functional Versatility in Tetrathiafulvalene-Lanthanide Metal-Organic Frameworks
}

\author{
Javier Castells-Gil, ${ }^{[a]}$ Samuel Mañas-Valero, ${ }^{[a]}$ Iñigo J. Vitórica-Yrezábal, ${ }^{[b]}$ Duarte Ananias, ${ }^{[c]}$ João \\ Rocha, ${ }^{[c]}$ Raul Santiago, ${ }^{[\mathrm{d}]}$ Stefan T. Bromley, ${ }^{[\mathrm{d}, \mathrm{e}]}$ José J. Baldoví, ${ }^{\left[{ }^{[]}\right]}$Eugenio Coronado, ${ }^{[\mathrm{a}]}$ Manuel \\ Souto*[a] and Guillermo Mínguez Espallargas*[a]
}

\begin{abstract}
Tetrathiafulvalene-Lanthanide (TTF-Ln) Metal-Organic Frameworks (MOFs) are an interesting class of multifunctional materials in which porosity can be combined with electronic properties such as electrical conductivity, redox activity, luminescence and magnetism. Herein we report a new family of isostructural TTF-Ln MOFs, denoted as MUV-5(Ln) (Ln = Gd, Tb, Dy, Ho, Er), exhibiting semiconducting properties as a consequence of the short intermolecular S...S contacts established along the chain direction between partially oxidised TTF moieties. In addition, this family shows photoluminescence properties and single-molecule magnetic behaviour, finding near-infrared (NIR) photoluminescence in the $\mathrm{Yb} / \mathrm{Er}$ derivative and slow relaxation of the magnetisation in the Dy and $\mathrm{Er}$ derivatives. As such properties are dependent on the electronic structure of the lanthanide ion, we emphasise the immense structural, electronic and functional versatility of this class of materials.
\end{abstract}

\section{Introduction}

During the last decades, the emergence of Metal-Organic Frameworks (MOFs), porous crystalline materials constructed from metallic nodes and organic linkers, has attracted a large attention in view of their limitless structural and functional versatility giving rise to a wide range of potential applications. ${ }^{[1]}$ Besides their intrinsic porosity, MOFs can incorporate electronic functionalities such electrical conductivity, ${ }^{[2,3]}$ magnetism ${ }^{[4]}$ and luminescence ${ }^{[5,6]}$ from their inorganic and organic building blocks. This permits the fabrication of novel multifunctional materials that

\footnotetext{
[a] J. Castells-Gil, S. Mañas-Valero, Prof. E. Coronado, Dr. M. Souto, Dr. G. Mínguez Espallargas

Instituto de Ciencia Molecular (ICMol), Universidad de Valencia

c/ Catedrático José Beltrán 2, 46980 Paterna, Spain

E-mail: manuel.souto@uv.es; guillermo.minguez@uv.es

[b] Dr. I. Vitórica-Yrezábal

School of Chemistry, University of Manchester

Oxford Road, Manchester M13 9PL, United Kingdom

[c] Dr. D. Ananias, Prof. J. Rocha

Department of Chemistry and CICECO-Aveiro Institute of Materials University of Aveiro

3810-193 Aveiro, Portugal

[d] Raul Santiago, Prof. S. T, Bromley

Departament de Ciència de Materials i Química Física \& Institut de Química Teòrica i Computacional, Universitat de Barcelona,

Universitat de Barcelona, C/Martí i Franquès 1, E-08028 Barcelona, Spain

[e] Prof. S. T, Bromley

Institució Catalana de Recerca i Estudis Avançats (ICREA), E08010 Barcelona, Spain

[f] Dr. J. J. Baldoví

Max Planck Institute for the Structure and Dynamics of Matter, Luruper Chaussee 149, D-22761 Hamburg, Germany
}

Supporting information for this article is given via a link at the end of the document. combine at the same time porosity with various physical properties. ${ }^{[7]}$

Tetrathiafulvalene(TTF)-based ligands are promising building-blocks for obtaining such materials since they can provide interesting features such as electrochemical properties or electrical conductivity. ${ }^{[8]}$ TTF derivatives show a remarkable electron-donor character and they can form $\pi-\pi$ stacks with relatively short $S \cdots S$ interactions to promote charge transport. For this reason, these systems have been widely studied as molecular conductors in the field of molecular electronics. In recent years, a variety of TTF-based MOFs have been reported to exhibit tunable electrical conductivity, ${ }^{[9,10]}$ photo-induced spin-crossover, ${ }^{[11]}$ enhanced catalytic activity ${ }^{[12]}$ or breathing-dependent redox activity. ${ }^{[13,14]}$

On the other hand, lanthanide Metal-Organic Frameworks (Ln-MOFs) have also attracted a broad interest since they can exhibit interesting structural and chemical properties including magnetism and luminescence. ${ }^{[15]}$ For example, trivalent lanthanide $\left(\mathrm{Ln}^{3+}\right)$ MOFs exhibiting photoluminescence are promising materials with numerous applications for the development of sensors and light-emitting devices. ${ }^{[5,6]}$ In addition, the incorporation of single-molecule magnetic (SMM) behaviour into a family of Ln-MOFs has been recently accomplished through the use of anisotropic magnetic ions ${ }^{[16,17]}$ making these materials very interesting candidates for quantum computation and molecular spintronic applications ${ }^{[18]}$.

In the last years, Pointillart et al. have described a variety of lanthanide coordination complexes bearing different TTF ligands which can exhibit multiple functionalities such as luminescence, redox properties and SMM behaviour. ${ }^{[19-21]}$ In addition to their intrinsic redox properties, TTF-based ligands can act as both structural agent and organic chromophore isolating the magnetic centres and sensitising the lanthanide luminescence. ${ }^{[20]}$ In this context, the three-dimensional organisation of these TTF-Ln complexes is encouraged in order to incorporate further functional properties such as electrical conductivity taking advantage of intermolecular interactions between partially oxidised TTF

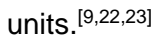

Along this line, two different TTF-Ln MOFs families exhibiting semiconducting and magnetic properties have been very recently reported. ${ }^{[23,24]}$ However, the crystal structures of these materials show the formation of orthogonal TTF dimers or large intermolecular S...S distances, which are usually problematic for attaining a proper orbital overlap between the TTF moieties and, thus, limiting the charge delocalization. Herein, we present a family of isostructural TTF-Ln MOFs, achieved by an alternative synthetic procedure and named as MUV-5(Ln) (MUV: Materials of University of Valencia; $\mathrm{Ln}=\mathrm{Gd}$, Tb, Dy, Ho, Er), yielding a new crystal structure in which the TTF moieties are arranged in a parallel fashion and with relatively short $S \cdots S$ interactions enhancing the electronic coupling between TTF units, as 
confirmed by transfer integral calculations. The TTF linkers are partially oxidised being responsible for charge transport and for the semiconducting behaviour of the MOF. In addition, photoluminescence properties and SMM behaviour have been studied for MUV-5. Such properties are dependent on the electronic nature of the lanthanide ion ${ }^{[26]}$, evidencing a wide structural, electronic, and functional versatility for this new family of TTF-Ln MOFs.

\section{Results and Discussion}

\section{Synthesis}

MUV-5(Ln) was synthesised by means of an isoreticular approach through an adapted synthetic procedure described for the synthesis of MUV-4(Dy). ${ }^{[17]}$ Solvothermal reaction of $\mathrm{Ln}\left(\mathrm{OOCCH}_{3}\right)_{3}$ and TTF-tetrabenzoic acid $\left(\mathrm{H}_{4}\right.$ TTFTB) in a mixture of water and acetic acid at $170{ }^{\circ} \mathrm{C}$ for 12 hours allowed the formation of single-crystals of MUV-5 (Scheme 1). Two crystalline polymorphs were identified by Single-Crystal X-Ray Diffraction (SCXRD), namely MUV-5a and MUV-5b, of formulae $\left[\mathrm{Ln}_{3}(\mathrm{TTFTB})_{2}(\mathrm{OOCCH})_{3}(\mathrm{OH})\left(\mathrm{H}_{2} \mathrm{O}\right)\right] \cdot 2.5 \mathrm{H}_{2} \mathrm{O}^{[27]}$ and [ $\mathrm{Ln}(\mathrm{HTTFTB})$ $\left.\left(\mathrm{H}_{2} \mathrm{O}\right)\right] \cdot\left(\mathrm{CH}_{3} \mathrm{COOH}\right)$, respectively, whose formation depends on the amount of acetic acid used in the synthesis.
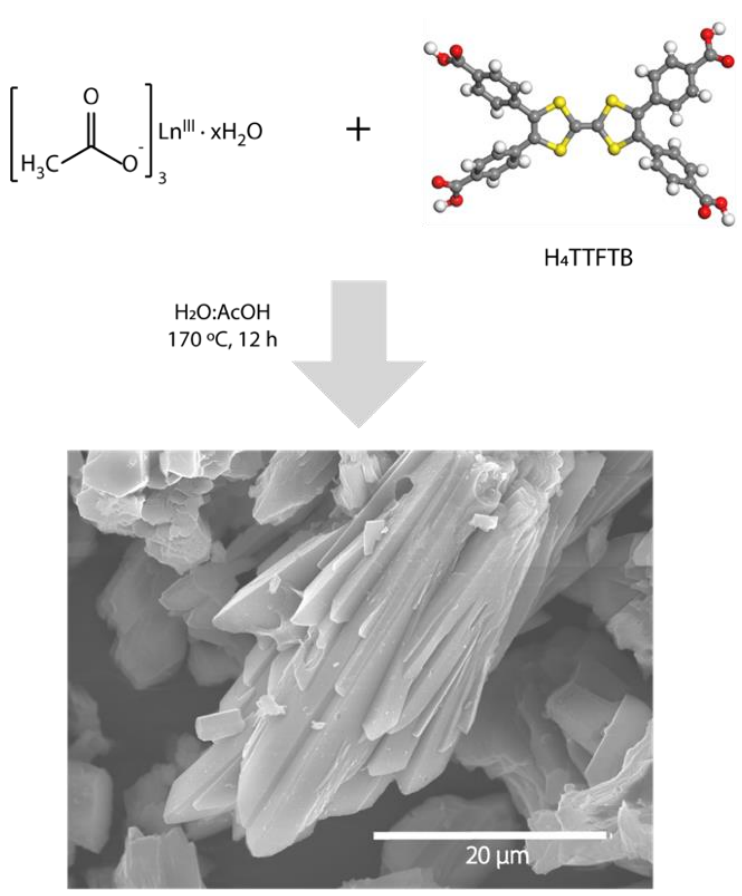

MUV-5

\section{Crystal structure}

MUV-5a crystallises in the monoclinic space group $P 2 / C$ (Tables $\mathrm{S} 1$ and S2). Two crystallographically independent $\mathrm{Ln}^{3+}$ ions are found in the asymmetric unit with different coordination environments. One of them is 7-coordinated with a capped trigonal prism geometry, whereas the second $\mathrm{Ln}^{3+}$ ion can be either 8 or 9-coordinated with a distorted triangular dodecahedron environment and spherical capped square antiprism, respectively, as calculated by the software SHAPE ${ }^{[25]}$ (Figures S1 and S2). The difference between the 8 or 9 -coordinated form in the latter relies on the position of the disordered acetate anion acting as a bridge between both $\mathrm{Ln}^{3+}$ ions (Figure $1 \mathrm{~b}$ ). The metallic secondary building unit (SBU) is composed of $\mathrm{Ln}^{3+}$ ions connected through bridging carboxylate bonds from the linker and acetate anions to form infinite Ln-carboxylate chain along the a-axis. The connection of these infinite Ln-carboxylate chains with the TTFTB linkers yields a 3D structure with two types of parallel 1D micropores extending along the a-axis that are filled with water molecules or coordinated acetate anions (Figure 1).

a)

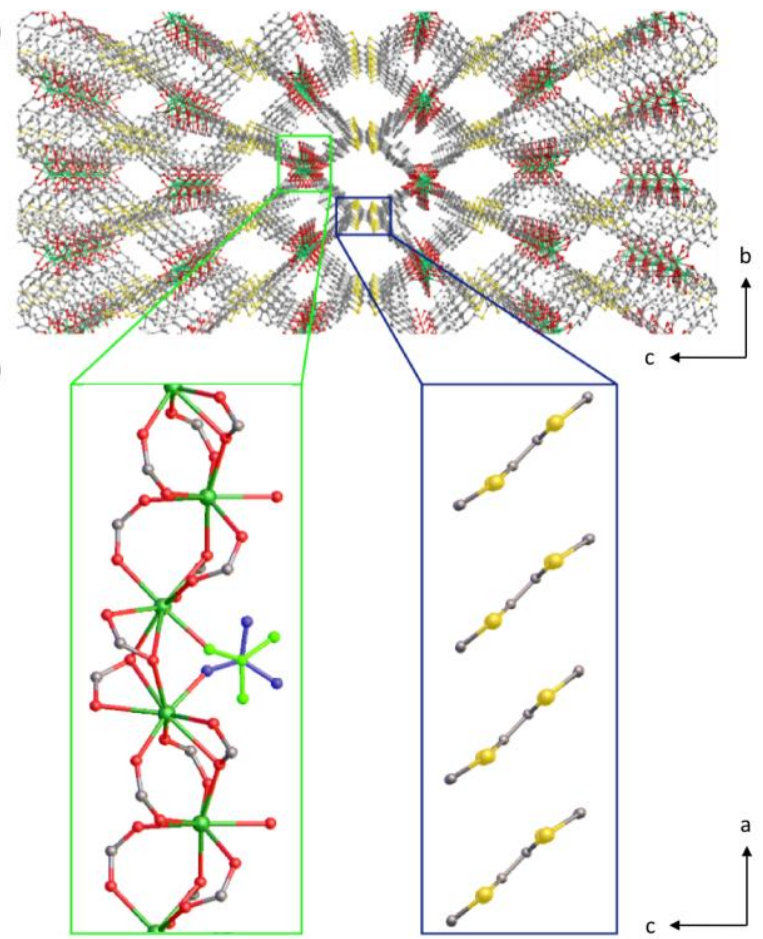

Figure 1. a) View of the crystal structure of MUV-5a along the $a$-axis and b) view of the arrangement of the $\mathrm{Ln}^{3+}$ ions (left) and TTF units (right) within the structure of MUV-5a. Colour code: C (grey), O (red), Ln (green), S (yellow). The disordered acetate anions have been highlighted as green and blue in order to show the two different orientations of the acetate molecules.

On the other hand, MUV-5b crystallizes in the monoclinic space group $P 2_{1} / n$. Unlike MUV-5a, MUV-5b has a dimeric $L n_{2}$ SBU similar to that observed in MUV-4a ${ }^{[17]}$. In this case, only one crystallographically independent $\mathrm{Ln}^{3+}$ ion is observed in the 
asymmetric unit. Each Ln atom is 8-coordinated with a distorted triangular dodecahedron geometry (Figures S3 and S4). The structure of MUV-5b is built upon dimeric SBUs of formula $\left[\mathrm{Ln}_{2}\left(\mathrm{H}_{2} \mathrm{O}\right)_{2}\left(\mu-\mathrm{O}_{2} \mathrm{C}\right)_{2}\left(\mathrm{O}_{2} \mathrm{C}\right)_{4}\left(\mathrm{HO}_{2} \mathrm{C}\right)_{2}\right]$ (Figure S5) in which the $\mathrm{Ln}^{3+}$ ions are kept at a distance of $4.88 \AA$ connected by two bridging carboxylate groups. The coordination sphere of each $\mathrm{Ln}^{3+}$ ion is completed by other 5 oxygen atoms from 3 different carboxylate groups and one water molecule (Figure S5). Finally, structure of MUV-5b also displays two types of one-dimensional micropores filled with acetic acid (Figure S3).

Pure crystalline phase of MUV-5a was confirmed by Powder X-Ray diffraction (PXRD, Figures S7-S12) and Thermogravimetric Analysis (TGA) (Figure S13). LeBail refinement converged with very good residual values $\left(\mathrm{R}_{\mathrm{wp}}=\right.$ $5.58 \% ; R_{p}=4.36 \%$, Figure S8, Table S3). The small onedimensional micropores allow the structure to have a free volume of $24 \%$ of the unit cell volume as calculated by Mercury ${ }^{[28]}$ (Figure S14). In order to examine the porosity of these materials, gas sorption experiments were performed on MUV-5a which was previously activated at $150 \stackrel{\circ}{\circ}$ under vacuum overnight. $\mathrm{N}_{2}$ adsorption-desorption isotherms performed at $77 \mathrm{~K}$ reveals a minimum uptake of $\mathrm{N}_{2}$, in line with other previously reported TTFbased coordination polymers. ${ }^{[29]}$ Interestingly, MUV-5a is able to adsorb $\mathrm{CO}_{2}$ with a modest $\mathrm{CO}_{2}$ uptake of $65 \mathrm{~cm}^{3} \cdot \mathrm{g}^{-1}$ (12.8 wt. \%) at $273 \mathrm{~K}$ (Figure S15), which is higher than that observed in other similar TTF-based MOFs at $195 \mathrm{~K}^{[29]}$, and a total uptake of 2.1 $\mathrm{mmol} \cdot \mathrm{g}^{-1}$ at 12 bar and $273 \mathrm{~K}$ (Figure 2). The selectivity of MUV5a towards the adsorption of $\mathrm{CO}_{2}$ over $\mathrm{N}_{2}$ likely arises from the higher quadrupole moment of $\mathrm{CO}_{2}$, which makes it capable of interacting more strongly with the framework. ${ }^{[29,30]}$ This feature opens the way for the utilization of this type of materials in gas separation or sensing applications.

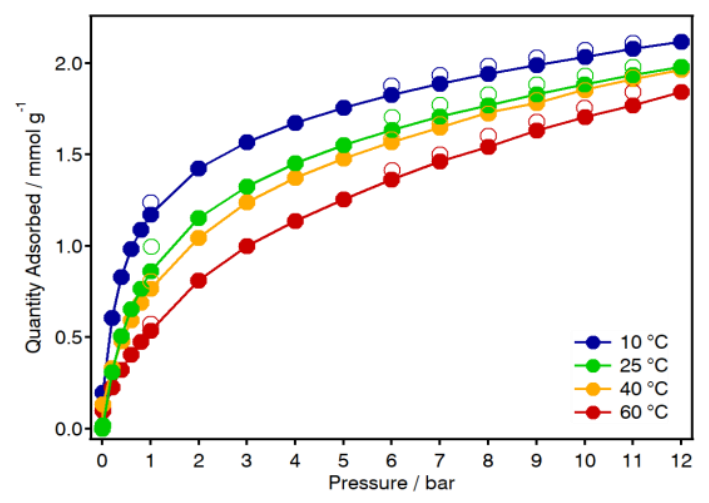

Figure 2. Adsorption (closed symbols) and desorption (open symbols) isotherms of $\mathrm{CO}_{2}$ at different temperatures in MUV-5a(Dy).

As far as the arrangement of the organic linkers is concerned, we note that in MUV-5 solids the TTF units are forming onedimensional segregated stacks of slightly slipped moieties running along the a-axis (Figures 1 and 3). However, there are important differences in both structures. First, the TTF struts in MUV-5b display a boat conformation with a central $\mathrm{C}=\mathrm{C}$ distance of $1.32 \AA$ and the dihedral angle between the planes formed by the two dithiol rings is $17^{\circ}$ (Figure S6), suggesting that the TTF moiety is in the neutral state ${ }^{[31,32]}$ (Figure 3a). On the other hand, the TTF moieties are packed along the $a$-axis with inter-centroid and $\mathrm{S} \cdots \mathrm{S}$ distances ranging from 4.40-4.90 $\AA$ and 3.92-4.27 $\AA$, respectively. In contrast, in MUV-5a the TTF units adopt an Sshaped conformation with a central $\mathrm{C}=\mathrm{C}$ bond distance of $1.34 \AA$, which is slightly longer than that in MUV-5b (Figure 3) and a dihedral angle of $5^{\circ}$, suggesting that the TTF units in MUV-5a are in the radical cation state (i.e. as $\mathrm{TTF}^{\cdot+}$ ), or at least partially oxidised. It is important to note that neutral TTF has a boat-like conformation $\left(C_{2 v}\right.$ symmetry) whereas $\mathrm{TTF}^{\cdot+}$ moieties have a planar $D_{2 \mathrm{~h}}$ symmetry as a result of the $6 \pi$-electron heteroaromaticity of the 1,2-dithiolium cation. ${ }^{[32,35]}$ The partial oxidation of the TTF units in MUV-5a was confirmed by EPR measurements of the crystals at room temperature that show a signal with a $g=2.007$, characteristic of organic radicals, and much more intense than the one for MUV-5b (Figure S16). As it has been observed in similar systems, TTF moieties in MUV-5a were at least partially oxidised during the MOF synthesis. ${ }^{[9]}$ We hypothesise that this excess of positive charge is compensated through deprotonation of coordinated water molecules. Otherwise, the TTF units are stacked in a parallel manner nearly equally separated with inter-centroid distances ranging from 4.06 to $4.28 \AA$ and being the closest S...S distances 3.61 and $3.86 \AA$ (Figure $3 b$ ). These distances between the TTF units in MUV-5a are significantly shorter than in MUV-5b, and comparable to other TTF-based MOFs that display electrical conductivity. ${ }^{[9,10]}$ This different arrangement with shorter intermolecular interactions between the partially oxidised TTF units in MUV-5a should be translated in a better orbital overlap between the linkers and, thus, in an enhancement in the conducting properties of the material that will be discussed below. 
a MUV-5a
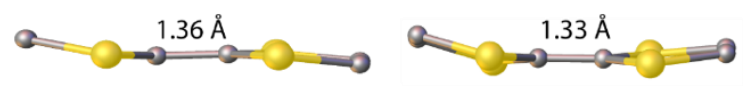

b
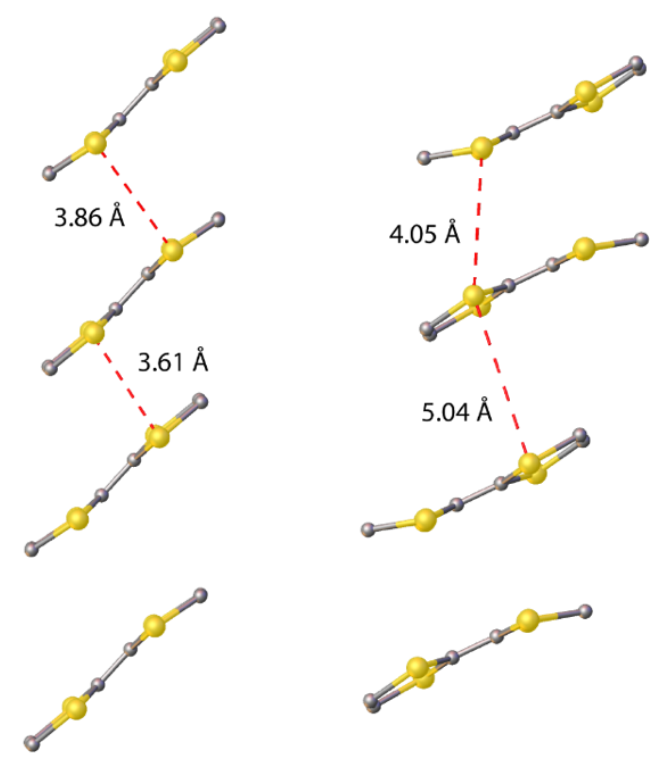

Figure 3. View of the a) conformation and b) arrangement of the TTF units within the structure of MUV-5a and MUV-5b. The red dashed lines show the shortest S $\cdots$ S distances between TTF pairs in MUV-5(Dy).

\section{Electrical conductivity}

In view of the formation of parallel stacks and partial oxidation of the TTF linkers which could promote charge transport in MUV$5 a(L n)$, transport measurements of the different samples were performed using four-contact probe pressed pellet devices from two independent batches (see Supporting Information for more information). The room temperature conductivities for MUV$5 \mathrm{a}(\mathrm{Gd})$, MUV-5a(Tb), MUV-5a(Dy), MUV-5a(Ho) and MUV$5 \mathrm{a}(\mathrm{Er})$ were found to be in the order of $10^{-5}-10^{-7} \mathrm{~S} \cdot \mathrm{cm}^{-1}$ (see Table S4 and Figures S18-S29). Although ground boundaries in pressed pellets induce large resistance in comparison to single crystal measurements, the obtained values are comparable to the ones for other TTF-based MOFs also measured as pressed pellets $^{[33]}$ and around two orders of magnitude higher than those for a recently reported TTF-Ln MOF family which show similar electrochemical properties (Figure S30). ${ }^{[25]}$ It is important to note that in our case electrical conductivity is achieved without the need of any doping process since TTF moieties are already partially oxidised during the synthesis, thus maintaining permanent porosity in the structures. On the other hand, electrical conductivity for MUV-5b(Dy) was measured to be in the order of $10^{-8} \mathrm{~S} \cdot \mathrm{cm}^{-1}$, one order of magnitude lower than for MUV-5a(Dy) which is consistent with the presence of larger S $\cdots S$ intermolecular distances and the absence of oxidised TTF units.
Charge values for the TTF moieties in each structure were estimated by taking into account the bond length $(\mathrm{C}=\mathrm{C} / \mathrm{C}-\mathrm{S})$ ratio ${ }^{[34]}$ obtaining values in the range from +0.1 to +0.7 suggesting that the TTF units are partially charged in MUV-5a (Table S5) in agreement with EPR spectroscopy. By contrast, the charge calculated in the case of MUV-5b indicate that TTF units are in the neutral state $(q=0)$. It should be noted that this correlation between charge and geometry is only approximate, although the estimated charges are in good agreement with the more planar conformation of the TTF units in MUV-5a (dihedral angle of $5^{\circ}$ ), which is typical for TTF $^{+\cdot}$ radical cation, whereas the boat-like conformation in MUV-5b (dihedral angle of $17^{\circ}$ ) is similar to the one for neutral TTF. ${ }^{[32,35]}$

\begin{tabular}{|c|c|c|}
\hline & Conductivity $\left(\mathrm{S} \cdot \mathrm{cm}^{-1}\right)^{[a]}$ & $\begin{array}{l}\text { Shortest S...S } \\
\text { distances }(\AA)\end{array}$ \\
\hline MUV-5a(Gd) & $2.0 \times 10^{-7}$ & 3.73 \\
\hline MUV-5a(Tb) & $1.5 \times 10^{-5}$ & 3.68 \\
\hline MUV-5a(Dy) & $3.9 \times 10^{-7}$ & 3.61 \\
\hline MUV-5a(Ho) & $6.7 \times 10^{-6}$ & 3.67 \\
\hline MUV-5a(Er) & $7.4 \times 10^{-6}$ & 3.65 \\
\hline MUV-5b(Dy) & $3.3 \times 10^{-7}$ & 4.05 \\
\hline
\end{tabular}

[a] Conductivities measured using four-contact probe pressed pellets at room temperature of two independent samples. Errors in the Supporting Information.

\section{Transfer integral calculations}

In order to further understand the difference of conductivities in MUV-5a and MUV-5b, DFT based calculations of intermolecular transfer integrals for TTF units in MUV-5 were performed. Transfer or hopping integrals $(t)$ measure the overlap of the frontier orbitals (i.e. HOMOs or LUMOs) between adjacent molecules and thus provide an indication of the strength of intermolecular conduction pathways for electrons (for HOMOs) or holes (for LUMOs). These calculations were performed using the Gaussian09 package ${ }^{[36]}$ employing the PBE0 [37] and B3LYP [38] hybrid functionals, with a $6-311+G(d, p)$ basis set. Transfer integrals between the TTF moieties in the MOFs were calculated in free space with no intervening metals at $0 \mathrm{~K}$ providing information about the overlap between the molecular orbitals of the TTF units through which charge transport is expected to occur. Calculations using both functionals provided consistent and comparable results (see Table S6) and we report B3LYP values hereafter. The transfer integrals for neutral MUV-5a were calculated to be $t_{1}=90.6$ and $t_{2}=16.5 \mathrm{meV}$ which are considerably higher than the ones for MUV-5b $\left(t_{1}=60.5\right.$ and $t_{2}=$ $8.4 \mathrm{meV}$ ) (Figure 4) in agreement with the higher conductivity measured for MUV-5a. We have also compared these values with the ones calculated for the other two TTF-Ln systems reported by Dincă ${ }^{[24]}$ and Zuo ${ }^{[25]}$ in order to examine potential correlations between the calculated transfer integrals and measured electrical conductivities. Regarding the systems reported by Dincă, ${ }^{[24]}$ the 
transfer integral was calculated to be $86.1 \mathrm{meV}$ between the TTF dimers. However, the transfer integrals are significantly lower (32.9 and $12.9 \mathrm{meV}$ ) between orthogonally displaced nondimerised TTF units (see Figure S31) in this system. On the other hand, although in the family of materials reported by Zuo and coworkers $^{[25]}$ the TTF units are displaced in a parallel fashion, the transfer integrals are lower (61.5 and $48.6 \mathrm{meV})$ than those for MUV-5a. This difference could help to explain the lower electrical conductivity reported by Zuo et al. $\left(10^{-8}-10^{-9} \mathrm{~S} \cdot \mathrm{cm}^{-1}\right)$ as compared to that measured for MUV-5a $\left(10^{-6}-10^{-7} \mathrm{~S} \cdot \mathrm{cm}^{-1}\right)$. a)

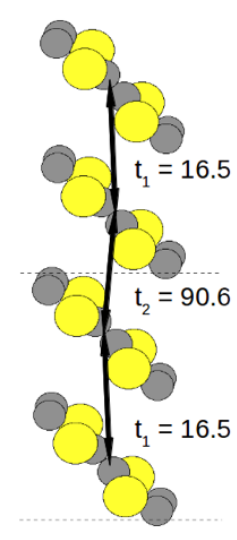

b)

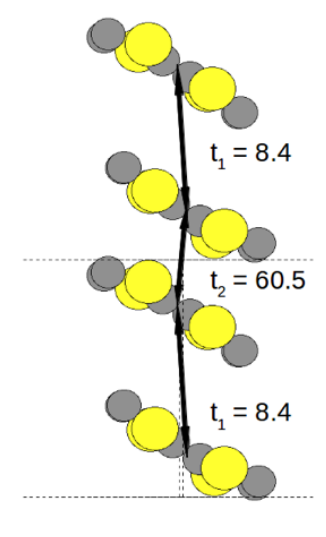

Figure 4. Schematic representation of TTF arrangements in the crystal structures of a) MUV-5a and b) MUV-5b indicating the transfer integrals (in meV).

\section{Photoluminescence properties}

To analyse the possible enhancement of photoluminescence via energy transfer among different lanthanide ions, ${ }^{[39]}$ the mixedlanthanide $\mathbf{M U V - 5 a}\left(\mathbf{Y b}_{0.76} \mathrm{Er}_{0.24}\right)$ was synthesized and characterized (see Supporting Information). The excitation spectra of MUV-5a(Ybo.76 $\left.E_{0.24}\right)$, MUV-5a(Er) and MUV-5a(Gd) compounds recorded at $295 \mathrm{~K}$ and $12 \mathrm{~K}$ (Figure 5) display a broad band from 250 to ca. $675 \mathrm{~nm}$ ascribed to the TTFTB ${ }^{4-}$ organic linker, covering most of the ultraviolet and visible spectral range. The spectra of MUV-5a ( $\left.\mathbf{Y b}_{\mathbf{0}} .76 \mathrm{Er}_{\mathbf{0 . 2 4}}\right)$ further display intense sharp lines in the 920-980 nm range assigned to the ${ }^{2} \mathrm{~F}_{7 / 2} \rightarrow{ }^{2} \mathrm{~F}_{5 / 2}$ transition of $\mathrm{Yb}^{3+}$. The poorer signal-to-noise ratio of $\mathrm{MUV}-5 \mathrm{a}(\mathrm{Er})$ sample spectra indicate that TTFTB ligand is more suited to sensitize $\mathrm{Yb}^{3+}$ than the $\mathrm{Er}^{3+}$ via the energy transfer process known as 'antenna effect'. Moreover, the $\mathrm{Yb}^{3+}$ emission may also be activated by direct excitation.

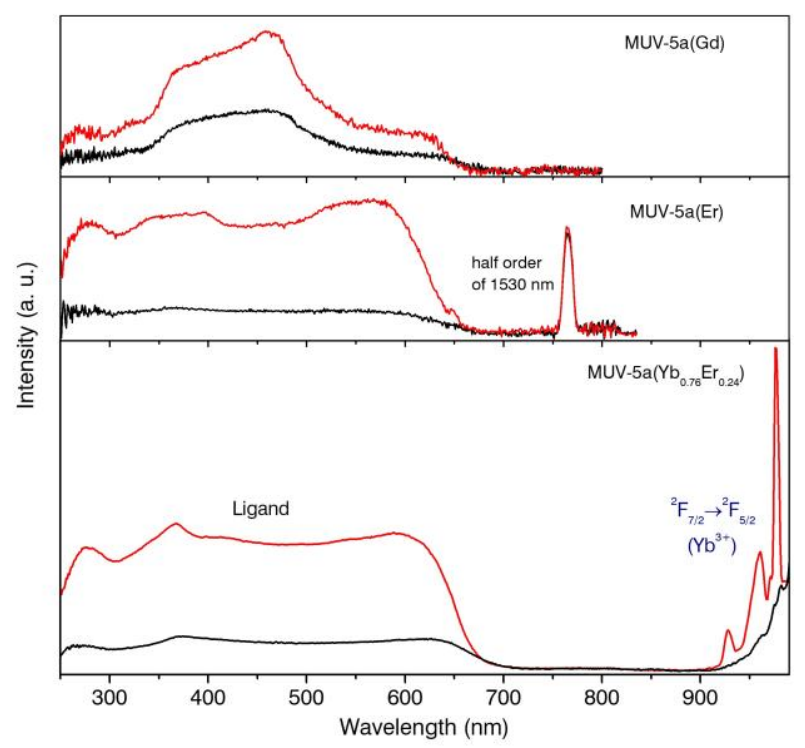

Figure 5. Excitation spectra of MUV-5a $\left(\mathbf{Y b}_{0.76} \mathrm{Er}_{0.24}\right)\left(\lambda_{\mathrm{Em}}=1040 \mathrm{~nm}\right)$, MUV-5a(Er) $\left(\lambda_{E m} .=1530 \mathrm{~nm}\right)$ and MUV-5a(Gd) $\left(\lambda_{\mathrm{Em} .}=1020 \mathrm{~nm}\right)$ recorded at $295 \mathrm{~K}$ (black lines) and at $12 \mathrm{~K}$ (red lines).

Upon ligand-mediated excitation at $560 \mathrm{~nm}, \mathbf{M U V}-5 \mathrm{a}\left(\mathbf{Y b}_{0.76} \mathrm{Er}_{0.24}\right)$ (Figure 6) shows both intense emission in the $980-1050 \mathrm{~nm}$ region assigned to the $\mathrm{Yb}^{3+}{ }^{2} \mathrm{~F}_{5 / 2} \rightarrow{ }^{2} \mathrm{~F}_{7 / 2}$ transition, and faint emission peaking at $1530 \mathrm{~nm}$ assigned to the $\mathrm{Er}^{3+}{ }^{4} \mathrm{I}_{13 / 2} \rightarrow{ }^{4} \mathrm{I}_{15 / 2}$ transition. At $12 \mathrm{~K}$, the well-defined sharp Stark components, resulting from the crystal-field splitting of the emitting states, demonstrate the crystallinity of the sample. Under the same excitation conditions, the emission of MUV-5a(Er) (Figure 6) is clearly weaker and dominated by a broad band ranging from ca. 940 to $1250 \mathrm{~nm}$; the $\mathrm{Er}^{3+} \mathrm{I}_{13 / 2} \rightarrow{ }^{4} I_{15 / 2}$ transition is only prominent at $12 \mathrm{~K}$. Because it is also present in the MUV-5a(Gd) spectra (Figure 6), the former band is attributed to the ligand's emission. The effective sensitization of the $\mathrm{Yb}^{3+}$ over the $\mathrm{Er}^{3+}$ emission is mainly due to a larger overlap between the ligand emission and the $\mathrm{Yb}^{3+}{ }^{2} \mathrm{~F}_{5 / 2}$ excited state, with an expected absorption cross-section at its maximum $(976 \mathrm{~nm})$ seven times larger than the resonant $980 \mathrm{~nm}$ $\mathrm{Er}^{3+}{ }^{4} \mathrm{I}_{11 / 2}$ level. ${ }^{40]}$ The larger $\mathrm{Yb}^{3+}$ cross-section is often used to improve the $\mathrm{Er}^{3+}$ emission via $\mathrm{Yb}^{3+}$-to- $\mathrm{Er}^{3+}$ energy transfer, including in Ln-bearing MOFs. ${ }^{[41,42]}$ This is not observed for MUV$5 \mathrm{a}\left(\mathrm{Yb}_{0.76} \mathrm{Er}_{0.24}\right)$ presumably due to an effective quenching of the $\mathrm{Er}^{3+}$ emission prompted by the $\mathrm{C}-\mathrm{H}, \mathrm{N}-\mathrm{H}$ and $\mathrm{O}-\mathrm{H}$ high energy vibrations of the ligand and free water molecules. Such quenching effect is more relevant for the $\mathrm{Er}^{3+}$ emission because the energy gap between the corresponding emitting and fundamental level is significantly smaller, ca. $6510 \mathrm{~cm}^{-1}$ vs. $10200 \mathrm{~cm}^{-1}$ for $\mathrm{Er}^{3+}$ and $\mathrm{Yb}^{3+}$, respectively. The $295 \mathrm{~K}$ and $12 \mathrm{~K} \mathrm{Yb}^{3+}$ emission lifetime in MUV-5a( $\left.\mathbf{Y b}_{0.76} \mathrm{Er}_{0.24}\right)$ are $7 \pm 1$ and $12 \pm 1 \mu \mathrm{s}$, respectively (Figure S33 in the Supporting Information). 


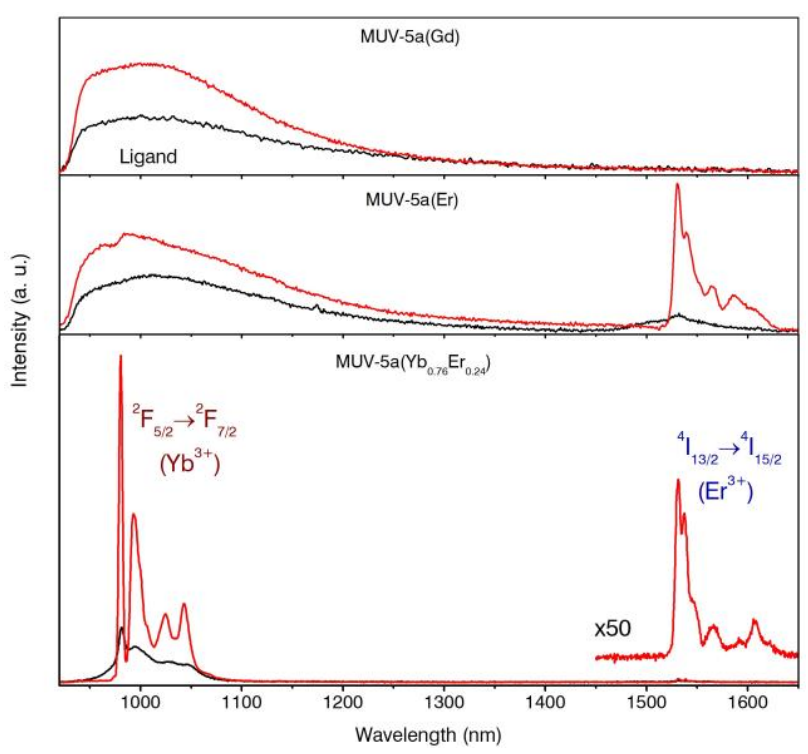

Figure 6. Emission spectra of MUV-5a(Ybo.76 $\left.E_{0.24}\right)\left(\lambda_{E x c .}=560 \mathrm{~nm}\right)$, MUV$5 \mathrm{a}(\mathrm{Er})\left(\lambda_{\mathrm{Exc} .}=560 \mathrm{~nm}\right)$ and MUV-5a(Gd) $\left(\lambda_{\mathrm{Exc} .}=430 \mathrm{~nm}\right)$ recorded at $295 \mathrm{~K}$ (black lines) and at $12 \mathrm{~K}$ (red lines).

\section{Magnetic properties}

Static magnetic measurements (dc) of MUV-5a solids were performed between 2 and $300 \mathrm{~K}$ under an applied field of $1000 \mathrm{G}$. The $X_{\mathrm{m}} \mathrm{T}$ values at room temperature of MUV-5a are close to those expected for the free $\mathrm{Ln}$ ion, which indicates that most of the energy levels are populated at room temperature (Figure 7). The drop observed in $X_{\mathrm{m}} \mathrm{T}$ at low temperatures is primarily ascribed to the depopulation of the highest energy levels, which is characteristic of anisotropic lanthanide ions. In case of MUV$5 \mathrm{a}(\mathrm{Er})$ there is a slight increase below $7 \mathrm{~K}$, which may indicate the presence of weak dipolar ferromagnetic interactions.

The static magnetic susceptibility was simulated using the Radial Effective Charge ${ }^{[43]}$ model, as implemented in the SIMPRE ${ }^{[44]}$ computational package (See ESI for details), for all the MUV-5a solids. The full set of crystal field parameters, ground$J$ multiplet energy level schemes and main contributions to the wave functions are reported in Tables S8-12. According to our calculations, the predicted ground state for MUV-5a(Dy1) is mainly composed by $\mid \pm 1 / 2>(98.7 \%)$, whereas MUV-5a(Dy2) is characterized by $80 \%$ of $\mid \pm 15 / 2>$. This means that, in the case of Dy, the second crystallographic centre is the one responsible of the observed SMM behaviour. This situation is dissimilar to MUV$5 \mathrm{a}(\mathrm{Er})$, where both crystallographic centres have a ground doublet characterized by high spin microstates $(80.1 \% \mid \pm 13 / 2>+$ $11.7 \% \mid \pm 15 / 2>$ in the case of Er1, and $66.6 \% \mid \pm 15 / 2>+19.0 \%$ $\mid \pm 9 / 2>$ for Er2). However, the energy difference between the ground state and the first excited state in both centres is $\sim 11$ and $25 \mathrm{~cm}^{-1}$, for Er1 and Er2, respectively, which together with molecular and lattice vibrations may limit the performance of these systems as molecular nanomagnets. ${ }^{[45]}$ On the other hand, the ground state of the different magnetic centres of MUV-5a(Tb) and MUV-5a(Ho) are mainly characterized by $\mid 0>$ or a heavy mixture in the case of Ho1, which suggests the presence of fast relaxation processes.



Figure 7. Experimental data (symbols) and theoretical simulation (lines) of the temperature dependence from 2 to $300 \mathrm{~K}$ of the magnetic susceptibility of MUV-5a. Tb (yellow), Dy (blue), Ho (purple) and Er (pink).

In order to demonstrate the differences in the magnetic behaviour of the different MUV-5a solids, we carried out dynamic (ac) magnetic measurements. As expected for MUV-5a(Tb) and MUV-5a(Ho), no out-of-phase signal ( $\left.X^{\prime \prime}\right)$ can be observed at frequencies as high as $1000 \mathrm{~Hz}$, even after applying an external dc magnetic field of $1000 \mathrm{G}$. In contrast, a frequency dependent out-of-phase signal is observed for MUV-5a(Dy) and MUV-5a(Er) as anticipated by the theoretical calculations (Figures S34 and S35). This is in agreement with the magnetic properties observed in similar Ln-MOFs. ${ }^{[25]}$ However, no maximum in the out-of-phase signal can be observed above $2 \mathrm{~K}$, likely due to the presence of a very fast relaxation of the magnetization through a quantum tunnelling mechanism, as it has been previously suggested for other MOFs showing SMM behaviour. ${ }^{[17]}$

\section{Conclusions}

In summary, we have reported a new family of isostructural TTFLn MOFs, denoted as MUV-5(Ln), which shows enhanced electrical semiconducting properties due to an effective 1D packing of the TTF moieties as confirmed by transfer integral calculations. In addition, near-infrared photoluminescence and slow relaxation of the magnetisation were investigated in different MUV-5 solids which can be finely tuned by suitable choice of the Ln ions, thus giving rise to a new family of versatile functional MOFs showing different properties within the same material at the same time. Current research is focused on the synergism between different physical properties, such as conductivity and luminescence, as well as on the influence of the trapped molecules on these physical properties. We are confident that our findings will help to broaden the family of MOF featuring tailored functionalities. 


\section{Experimental Section}

Synthesis of MUV-5(Ln): Common synthesis of MUV-5 solids were carried out by suspending $16.5 \mu \mathrm{mol}$ of $\mathrm{Ln}\left(\mathrm{CH}_{3} \mathrm{COO}\right)_{3} \cdot \mathrm{xH}_{2} \mathrm{O}$ and $12.5 \mu \mathrm{mol}$ of $\mathrm{H}_{4}$ TTFTB $^{[12]}$ in a mixture of $1.3 \mathrm{~mL}$ of $\mathrm{H}_{2} \mathrm{O}$ and $0.7 \mathrm{~mL}$ of $\mathrm{AcOH}$ (MUV-5a) or $1.5 \mathrm{~mL}$ of $\mathrm{H}_{2} \mathrm{O}$ and $0.5 \mathrm{~mL}$ of $\mathrm{AcOH}$ (MUV-5b) in a $4 \mathrm{~mL}$ glass vial. The vial was sealed and sonicated for a few minutes to get a homogeneous suspension. The dark suspension was subsequently heated in an oven at $170 \stackrel{\circ}{\circ}$ for 12 hours $\left(\uparrow+2.0^{\circ} \mathrm{C} \mathrm{min}{ }^{-1}, \downarrow-0.4{ }^{\circ} \mathrm{C} \mathrm{min}^{-1}\right)$. After cooling down to room temperature, the dark red crystals were recovered by centrifugation and rinsed with fresh DMF, water and $\mathrm{MeOH}$ several times. The solid was then allowed to dry in air at room temperature, and was further heated at $150{ }^{\circ} \mathrm{C}$ for at least 2 hours in order to yield activated MUV-5a. Anal. Elem. MUV-5a $\left[\mathrm{Dy}_{3}\left(\mathrm{C}_{34} \mathrm{H}_{16} \mathrm{O}_{8} \mathrm{~S}_{4}\right)_{2}\left(\mathrm{OOCCH}_{3}\right)\left(\mathrm{OCHN}\left(\mathrm{CH}_{3}\right)_{2}\right)\right] \cdot\left(\mathrm{OCHN}\left(\mathrm{CH}_{3}\right)_{2}\right)$ Calcd.: C, 44.44; H, 2.40; S, 12.49; N, 1.36. Found: C, 45.05; H, 2.68; $\quad \mathrm{S}, \quad$ 12.37.; $\quad \mathrm{N}, \quad 0.99$. MUV-5b $\left[\mathrm{Dy}\left(\mathrm{C}_{34} \mathrm{H}_{16} \mathrm{O}_{8} \mathrm{~S}_{4}\right)\left(\mathrm{H}_{2} \mathrm{O}\right)\right]\left(\mathrm{CH}_{3} \mathrm{COOH}\right]$ : Calcd: $\mathrm{C}, 46.93 ; \mathrm{H}, 2.41 ; \mathrm{S}$, 13.92. Found: C, 46.96; H, 2.67; S, 13.17.

General methods and materials, crystal data, chemical characterisation, electrical measurements, transfer integral calculations, photoluminescence, magnetic measurements and calculations are available in the Supporting Information.

\section{Acknowledgements}

This work has been supported by the European Union (ERC2016-CoG 724681-S-CAGE), by the Spanish MICINN (CTQ2017-89528-P and MATMAT2017-89993-R co-financed by FEDER) and by the project CICECO-Aveiro Institute of Materials (FCT Ref. UID/CTM/50011/2019) financed by FCT/MCTES. We also thank the Spanish government for the provision of a Maria de Maeztu project (MDM-2015-0538) and the Generalitat Valeciana (PROMETEO/2017/066). G.M.E., M.S. and S.M.V. thank MICINN for a Ramón y Cajal, Juan de la Cierva-Formación and FPU fellowships, respectively. J. J. B. thanks the EU for a Marie Skłodowska-Curie Fellowship (H2020-MSCA-IF-2016751047). We thank the Diamond Light Source (UK) for the synchrotron beamtime (MT17379). We thank Roger Sanchis for electrochemical measurements and Gloria Agustí and José M. Martínez-Agudo for magnetic measurements.

Keywords: Metal-Organic Framework • Tetrathiafulvalene • Lanthanide $\cdot$ Conductivity $\cdot$ Luminescence $\cdot$ Single-Molecule Magnet

[1] G. Maurin, C. Serre, A. Cooper, G. Férey, Chem. Soc. Rev. 2017 46, 3104-3107.

[2] L. Sun, M. G. Campbell, M. Dincă, Angew. Chem. Int. Ed. 2016, 55 3566-3579.

[3] I. Stassen, N. Burtch, A. Talin, P. Falcaro, M. Allendorf, R. Ameloot Chem. Soc. Rev. 2017, 46, 3185-3241.

[4] G. Mínguez Espallargas, E. Coronado, Chem. Soc. Rev. 2018, 533557.

[5] J. Rocha, L. D. Carlos, F. A. A. Paz, D. Ananias, Chem. Soc. Rev. 2011, 40, 926-940.

[6] W. P. Lustig, S. Mukherjee, N. D. Rudd, A. V. Desai, J. Li, S. K. Ghosh, Chem. Soc. Rev. 2017, 46, 3242-3285.

[7] B. Li, H. M. Wen, Y. Cui, W. Zhou, G. Qian, B. Chen, Adv. Mater. B. Li, H. M. Wen, Y. Cui,

[8] H.-Y. Wang, L. Cui, J.-Z. Xie, C. F. Leong, D. M. D’Alessandro, J.-L. Zuo, Coord. Chem. Rev. 2017, 345, 342-361. Voorhis, M. Dincă, J. Am. Chem. Soc. 2015, 137, 1774-1777. H.-Y. Wang, J.-Y. Ge, C. Hua, C.-Q. Jiao, Y. Wu, C. F. Leong, D. M. D’Alessandro, T. Liu, J.-L. Zuo, Angew. Chem. Int. Ed. 2017, 56, $5465-5470$.

M. Souto, A. Santiago-Portillo, M. Palomino, I. J. Vitórica-Yrezábal, B. J. C. Vieira, J. C. Waerenborgh, S. Valencia, S. Navalón, F. Rey, H. García, G. Mínguez Espallargas, Chem. Sci. 2018, 9, 2413-

2418.
J. Su, S. Yuan, H.-Y. Wang, L. Huang, J.-Y. Ge, E. Joseph, J. Qin, T. Cagin, J.-L. Zuo, H.-C. Zhou, Nat. Commun. 2017, 8, 2008. M. Souto, J. Romero, J. Calbo, I. J. Vitorica-Yrezabal, J. L. Zafra, J. Casado Cordón, E. Ortí, A. Walsh, G. Mínguez Espallargas, J. Am. Chem. Soc. 2018, 140, 10562-10569.

D. M. P. Mingos, Lanthanide Metal-Organic Frameworks, SpringerVerlag Berlin Heidelberg, 2015.

J. J. Baldoví, E. Coronado, A. Gaita-Ariño, C. Gamer, M. GiménezMarqués, G. Mínguez Espallargas, Chem. Eur. J. 2014, 20, 1069510702.

J. Castells-Gil, J. J. Baldoví, C. Martí-Gastaldo, G. Mínguez Espallargas, Dalt. Trans. 2018, 47, 14734-14740.

A. Gaita-Ariño, F. Luis, S. Hill, E. Coronado, Nat. Chem. 2019, 11 301-309.

F. Pointillart, B. Le Guennic, S. Golhen, O. Cador, L. Ouahab, Chem. Commun. 2013, 49, 11632-11634.

F. Pointillart, B. Le Guennic, O. Cador, O. Maury, L. Ouahab, Acc. Chem. Res. 2015, 48, 2834-2842.

F. Pointillart, J. Jung, R. Berraud-pache, B. Le Guennic, V. Dorcet, O. Cador, O. Maury, Y. Guyot, S. Decurtins, S. Liu, et al., Inorg. Chem. 2015, 54, 5384-5397.

M. Bendikov, F. Wudl, D. F. Perepichka, Chem. Rev. 2004, 104 4891-4945.

C. Rovira, Chem. Rev. 2004, 104, 5289-5317.

L. S. Xie, M. Dincǎ, Isr. J. Chem. 2018, 2139, 1119-1122.

J. Su, T. Hu, R. Murase, H. Wang, D. M. D. Alessandro, M. Kurmoo, J. Zuo, Inorg. Chem. 2019, 58, 3698-3706.

J. J. Baldoví, S. Cardona-Serra, J. M. Clemente-Juan, E. Coronado, A. Gaita-Ariño, A. Palii, Inorg. Chem. 2012, 51, 12565-12574. Formula of MUV-5a(Dy) is [Dy $(\text { TTFTB })_{2}\left(\mathrm{OOCCH}_{3}\right)\left(\mathrm{OCHN}\left(\mathrm{CH}_{3}\right)_{2}\right]$ having a coordinated DMF instead of $\mathrm{H}_{2} \mathrm{O} / \mathrm{OH}$ since these crystals were not properly washed and activated by heating.

C. F. Macrae, P. R. Edgington, P. McCabe, E. Pidcock, G. P. Shields, R. Taylor, M. Towler, J. van de Streek, J. Appl. Cryst. 2006 39, 453-457.

B. Chen, Z. P. Lv, C. F. Leong, Y. Zhao, D. M. D'alessandro, J. L. Zuo, Cryst. Growth Des. 2015, 15, 1861-1870.

B. Liu, B. Smit, Langmuir 2009, 25, 5918-5926. T. J. Kistenmacher, T. E. Phillips, D. O. Cowan, Acta Cryst. 1974, B30, 763-768.

V. Mukherjee, N. P. Singh, Spectrochim Acta A Mol Biomol Spectrosc 2014, 117, 315-322.

L. Sun, S. S. Park, D. Sheberla, M. Dincă, J. Am. Chem. Soc. 2016 138, 14772-14782.

[34] T. C. Umland, S. Allie, T. Kuhlmann, P. Coppens, J. Phys. Chem. 1988, 92, 6456-6460.

[35] S. Vela, M. Souto, I. Ratera, C. Rovira, J. Veciana, J. Phys. Chem. A 2016, 120, 10297-10303.

[36] M. J. Frisch, Gaussian 09, Revision B.01, Gaussian, Inc. Wallingford CT, 2016

[37] A. D. Becke, J. Chem. Phys. 1993, 98, 5648-5652.

[38] C. Adamo, V. Barone, J. Chem. Phys. 1999, 110, 6158-6170.

[39] S. Abednatanzi, P. Gohari Derakhshandeh, H. Depauw, F.-X Coudert, H. Vrielinck, P. Van Der Voort, K. Leus, Chem. Soc. Rev. 2019, 48, 2535-2565.

[40] C. Strohhöofer, A. Polman, Opt. Mater. 2013, 21, 705-712.

[41] K. A. White, D. A. Chengelis, K. A. Gogick, J. Stehman, N. L. Rosi, S. Petoud, J. Am. Chem. Soc. 2009, 131, 18069-18071.

[42] F. Artizzu, F. Quochi, L. Marchiò, E. Sessini, M. Saba, A. Serpe, A. Mura, M. L. Mercuri, G. Bongiovanni, P. Deplano, J. Phys. Chem. Lett. 2013, 4, 3062-3066.

[43] J. J. Baldoví, J. J. Borrás-Almenar, J. M. Clemente-Juan, E. Coronado, A. Gaita-Ariño, Dalt. Trans. 2012, 41, 13705-13710.

[44] J. J. Baldoví, S. Cardona-Serra, J. M. Clemente-Juan, E. Coronado, A. Gaita-Ariño, A. Palii, J. Comput. Chem. 2013, 34, 1961-1967.

[45] L. Escalera-Moreno, J. J. Baldoví, A. Gaita-Ariño, E. Coronado, Chem. Sci. 2018, 9, 3265-3275. 


\section{Entry for the Table of Contents}

\section{FULL PAPER}

Herein we report a new family of isostructural TTF-Ln MOFs ( $\mathrm{Ln}=\mathrm{Gd}$, Tb, Dy, Ho, Er), named MUV-5, exhibiting electrical conductivity, NIR luminescence and magnetic properties.

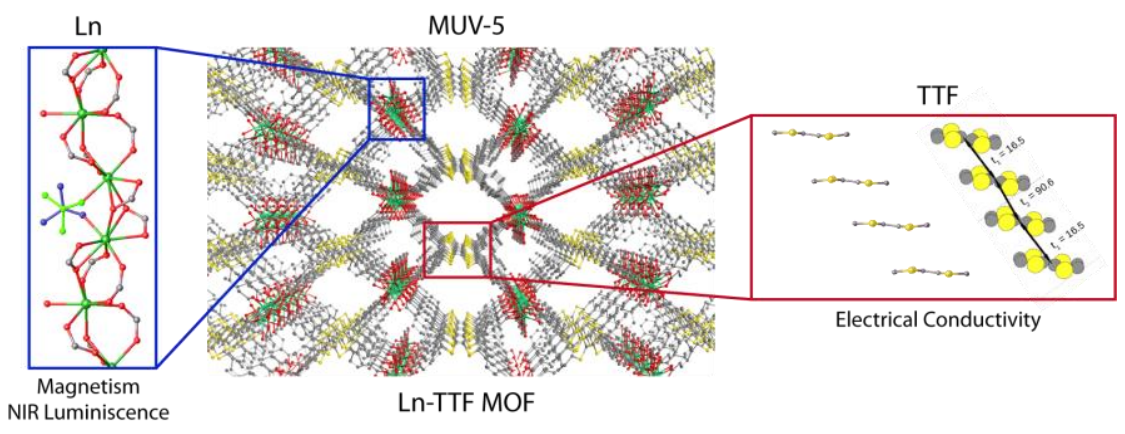

Javier Castells-Gil, Samuel MañasValero, Iñigo J. Vitórica-Yrezábal, Duarte Ananias, João Rocha, Raul Santiago, Stefan T. Bromley, José J. Baldoví, Eugenio Coronado, Manuel Souto* and Guillermo Mínguez Espallargas*

Page No. - Page No.

Electronic, Structural and Functional Versatility in TetrathiafulvaleneLanthanide Metal-Organic Frameworks 


\section{Electronic, Structural and Functional Versatility in Tetrathiafulvalene-Lanthanide Metal-Organic Frameworks}

Javier Castells-Gil, ${ }^{[a]}$ Samuel Mañas-Valero, ${ }^{[a]}$ Iñigo J. Vitórica-Yrezábal, ${ }^{[b]}$ Duarte Ananias, ${ }^{[c]}$ João Rocha, ${ }^{[\mathrm{c}]}$ Raul Santiago, ${ }^{[\mathrm{d}]}$ Stefan T. Bromley, ${ }^{[\mathrm{d}, \mathrm{e}]}$ José J. Baldoví, ${ }^{[\mathrm{f}]}$ Eugenio Coronado, ${ }^{\left[{ }^{[a]}\right.}$ Manuel Souto*[a] and Guillermo Mínguez Espallargas*[a]

a Universidad de Valencia (ICMol), Catedrático José Beltrán 2, 46980, Paterna (Spain)

${ }^{\mathrm{b}}$ School of Chemistry, University of Manchester Oxford Road, Manchester M13 9PL, United Kingdom

c Department of Chemistry and CICECO-Aveiro Institute of Materials University of Aveiro 3810-193 Aveiro, Portugal

${ }^{\mathrm{d}}$ Departament de Ciència de Materials i Química Física \& Institut de Química Teòrica i Computacional, Universitat de Barcelona, Universitat de Barcelona, C/Martí i Franquès 1, E-08028 Barcelona, Spain

${ }^{\mathrm{e}}$ Institució Catalana de Recerca i Estudis Avançats (ICREA), E-08010 Barcelona, Spain

${ }^{f}$ Max Planck Institute for the Structure and Dynamics of Matter, Luruper Chaussee 149, D-22761 Hamburg, Germany

\section{Table of contents}

S1. MATERIALS AND REAGENTS

S2. SYNTHESIS OF MATERIALS AND EXPERIMENTAL DETAILS.......................................................2

S3. CRYSTAL DATA AND STRUCTURE REFINEMENT FOR MUV-5(LN), ..........................................3

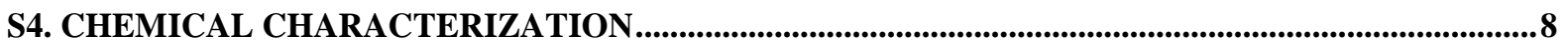

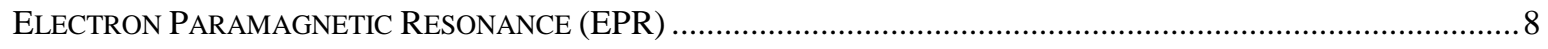

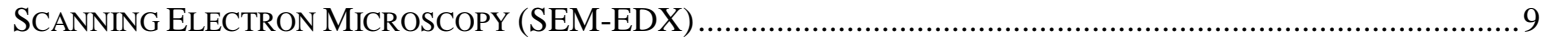

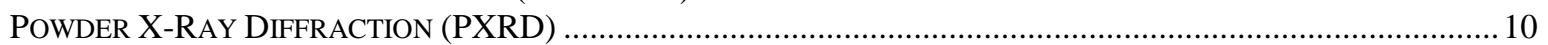

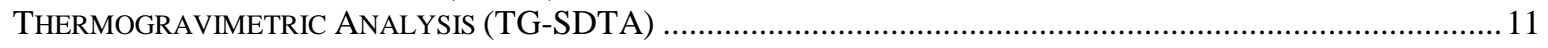

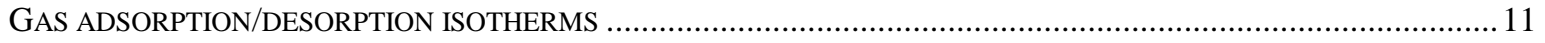

S5. ELECTRICAL MEASUREMENTS.........................................................................................14

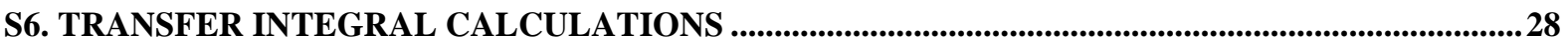

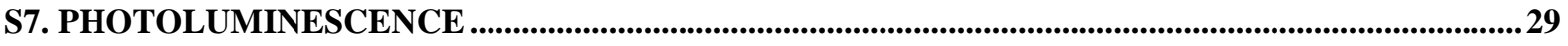

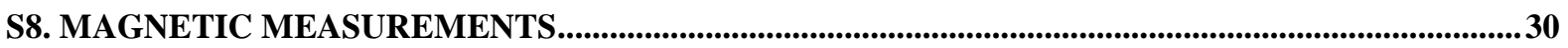

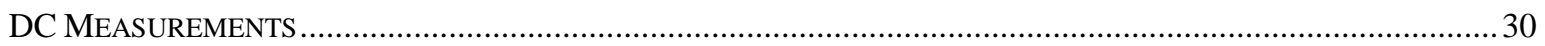

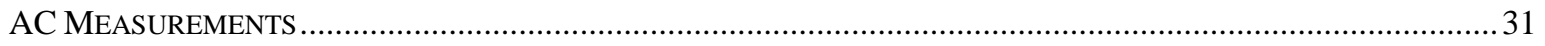

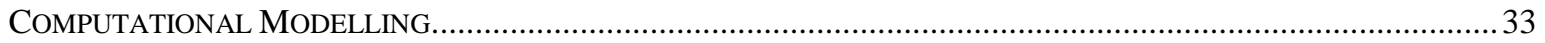

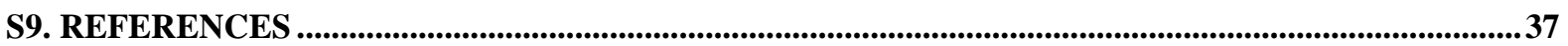




\section{S1. Materials and reagents.}

$\mathrm{Ln}\left(\mathrm{O}_{2} \mathrm{CCH}_{3}\right)_{3} \cdot \mathrm{xH}_{2} \mathrm{O}$ precursors were purchased from Sigma-Aldrich and TCI Chemicals. $N, N-$ dimethylformamide (DMF) (99.8\%) and acetic acid (99.7\%) were purchased from Sigma-Aldrich. Milli-Q water was used when required. All reagents and solvents were used as received without further purification.

\section{S2. Synthesis of materials and experimental details.}

Synthesis of MUV-5(Ln): Common synthesis of MUV-5 solids were carried out by suspending $16.5 \mu \mathrm{mol}$ of $\mathrm{Ln}\left(\mathrm{CH}_{3} \mathrm{COO}\right)_{3} \cdot \mathrm{xH}_{2} \mathrm{O}$ and $12.5 \mu \mathrm{mol}$ of $\mathrm{H}_{4}$ TTFTB in a mixture of $1.3 \mathrm{~mL}$ of $\mathrm{H}_{2} \mathrm{O}$ and $0.7 \mathrm{~mL}$ of AcOH (MUV5a) or $1.5 \mathrm{~mL}$ of $\mathrm{H}_{2} \mathrm{O}$ and $0.5 \mathrm{~mL}$ of $\mathrm{AcOH}$ (MUV-5b) in a $4 \mathrm{~mL}$ glass vial. The vial was sealed and sonicated for a few minutes to get a homogeneous suspension. The dark suspension was subsequently heated in an oven at $170{ }^{\circ} \mathrm{C}$ for 12 hours $\left(\uparrow+2.0{ }^{\circ} \mathrm{C} \mathrm{min}{ }^{-1}, \downarrow-0.4{ }^{\circ} \mathrm{C} \mathrm{min}-1\right)$. After cooling down to room temperature, the dark red crystals were recovered by centrifugation and rinsed with fresh $\mathrm{DMF}$, water and $\mathrm{MeOH}$ several times. The solid was then allowed to dry in air at room temperature, and was further heated at $150{ }^{\circ} \mathrm{C}$ for at least 2 hours in order to yield activated MUV-5a. Anal. Elem. MUV-5a $\left.\left[\mathrm{Dy}_{3}\left(\mathrm{C}_{34} \mathrm{H}_{16} \mathrm{O}_{8} \mathrm{~S}_{4}\right)_{2}\left(\mathrm{OOCCH}_{3}\right)\left(\mathrm{OCHN}_{(} \mathrm{CH}_{3}\right)_{2}\right)\right]$. $\left(\mathrm{OCHN}\left(\mathrm{CH}_{3}\right)_{2}\right)$ : Calcd.: C, 44.44; H, 2.40; S, 12.49; N, 1.36. Found: C, 45.33; H, 2.68; S, 12.37.; N, 0.99. MUV5b $\left[\mathrm{Dy}\left(\mathrm{C}_{34} \mathrm{H}_{16} \mathrm{O}_{8} \mathrm{~S}_{4}\right)\left(\mathrm{H}_{2} \mathrm{O}\right)\right]\left(\mathrm{CH}_{3} \mathrm{COOH}\right]$ : Calcd.: C, 46.93; H, 2.41; S, 13.92. Found: C, 46.96; H, 2.67; S, 13.17. 


\section{S3. Crystal data and structure refinement for MUV-5(Ln).}

Data Collection. X-ray data for compounds MUV-5a(Dy) and MUV-5a(Ho) were collected at a temperature of $150 \mathrm{~K}$ using Rigaku FR-X with $\mathrm{Cu}-\mathrm{K} \alpha$ radiation equipped with a HypixHE6000 detector. X-ray data for compounds MUV-5a(Er), MUV-5a(Gd) and MUV-5a(Tb) were collected at a temperature of $100 \mathrm{~K}$ at I19 beamline at Diamond Light Source synchrotron. ${ }^{1}$ X-ray data for compound MUV-5b(Dy) were collected at a temperature of $120 \mathrm{~K}$ using a Rigaku Supernova with Mo-K $\alpha$ radiation equipped with an Eos CCD detector. All instruments were equipped with an Oxford Cryosystems nitrogen flow gas system. Data was measured using GDA for MUV-5a(Er) and MUV-5a(Tb) and CrysAlisPro suite of programs for MUV-5a(Dy), MUV-5a(Ho) and MUV-5b(Dy).

Crystal structure determinations and refinements. X-ray data were processed and reduced using CrysAlisPro suite of programs. Absorption correction was performed using empirical methods (SCALE3 ABSPACK) based upon symmetry-equivalent reflections combined with measurements at different azimuthal angles. ${ }^{2}$ The crystal structure was solved and refined against all $F^{2}$ values using the SHELXL and Olex 2 suite of programmes. ${ }^{3.4}$

All atoms in crystal structures were refined anisotropically with the exception of some water solvent molecules. Hydrogen atoms were placed in the calculated idealized positions for all compounds. Disordered water molecules and the hydrogen bond network produced suggest the possibility of having a disordered $\mathrm{H}_{2} \mathrm{O} / \mathrm{OH}$ (50/50) coordinated to the metal atoms in compounds MUV-5a(Ho), MUV-5a(Er), MUV-5a(Gd) and MUV$5 \mathrm{a}(\mathrm{Tb})$. All compounds present large voids filled with a lot of featureless electron density; solvent mask protocol implemented by Olex 2 show the presence of 80 electrons approximately in the voids, which could correspond with 2 molecules of DMF per void.

CCDC 1934945-1934950 contains the supplementary crystallographic data for this paper. These data can be obtained free of charge via www.ccdc.cam.ac.uk/conts/retrieving.html (or from the Cambridge Crystallographic Data Centre, 12 Union Road, Cambridge CB21EZ, UK; fax: (+44)1223-336-033; or deposit@ccdc.cam.ac.uk).

Table S1. Crystallographic information for MUV-5a(Dy), MUV-5b(Dy) and MUV-5a(Er)

\begin{tabular}{|c|c|c|c|}
\hline Identification code & MUV-5a(Dy) & MUV-5b(Dy) & MUV-5a(Er) \\
\hline Empirical formula & $\mathrm{C}_{73} \mathrm{H}_{42} \mathrm{Dy}_{3} \mathrm{NO}_{19} \mathrm{~S}_{8}$ & $\mathrm{C}_{36} \mathrm{H}_{23} \mathrm{DyO}_{11} \mathrm{~S}_{4}$ & $\mathrm{C}_{70} \mathrm{H}_{41.5} \mathrm{Er}_{3} \mathrm{O}_{21.5} \mathrm{~S}_{8}$ \\
\hline Formula weight & 1981.05 & 922.28 & 1984.79 \\
\hline Temperature/K & $150(2)$ & $120(2)$ & $100(2)$ \\
\hline Crystal system & monoclinic & monoclinic & monoclinic \\
\hline Space group & $P 2 / c$ & $P 2_{1} / n$ & $P 2 / c$ \\
\hline $\mathrm{a} / \AA ̊$ & $11.1878(9)$ & $20.8560(4)$ & $11.1440(2)$ \\
\hline $\mathrm{b} / \AA$ & $11.0780(9)$ & $10.30930(10)$ & $11.3378(2)$ \\
\hline $\mathrm{c} / \AA$ & $32.841(2)$ & $21.1597(4)$ & $32.2847(4)$ \\
\hline$\alpha /^{\circ}$ & 90 & 90 & 90.0 \\
\hline$\beta /{ }^{\circ}$ & $91.771(7)$ & $116.201(2)$ & $91.3192(14)$ \\
\hline$\gamma /{ }^{\circ}$ & 90 & 90 & 90 \\
\hline Volume $/ \AA^{3}$ & $4068.3(6)$ & $4082.10(13)$ & 4078.04(11) \\
\hline $\mathrm{Z}$ & 2 & 4 & 2 \\
\hline$\rho_{\text {calc }} \mathrm{g} / \mathrm{cm}^{3}$ & 1.617 & 1.501 & 1.616 \\
\hline$\mu / \mathrm{mm}^{-1}$ & 16.980 & 2.089 & 3.325 \\
\hline $\mathrm{F}(000)$ & 1930.0 & 1828.0 & 1931.0 \\
\hline Crystal size $/ \mathrm{mm}^{3}$ & $0.03 \times 0.012 \times 0.01$ & $0.18 \times 0.04 \times 0.01$ & $0.018 \times 0.01 \times 0.01$ \\
\hline Radiation & $\mathrm{CuK} \alpha(\lambda=1.54184)$ & $\operatorname{MoK} \alpha(\lambda=0.71073)$ & synchrotron $(\lambda=0.6889)$ \\
\hline $2 \Theta$ range for data collection ${ }^{\circ}$ & 5.384 to 133.194 & 6.854 to 56.204 & 3.482 to 49.038 \\
\hline Index ranges & $-13 \leq \mathrm{h} \leq 12,-11 \leq \mathrm{k} \leq 13,-38 \leq 1 \leq 39$ & $\begin{array}{c}-27 \leq \mathrm{h} \leq 26,-13 \leq \mathrm{k} \leq 13,-26 \leq 1 \\
\leq 27\end{array}$ & $\begin{array}{c}-13 \leq \mathrm{h} \leq 12,-13 \leq \mathrm{k} \leq 12,-38 \leq 1 \\
\leq 38\end{array}$ \\
\hline Reflections collected & 22453 & 45547 & 27237 \\
\hline Independent reflections & $6883\left[\mathrm{R}_{\mathrm{int}}=0.0771, \mathrm{R}_{\mathrm{sigma}}=0.0681\right]$ & $\begin{array}{c}9020\left[R_{\text {int }}=0.0678, R_{\text {sigma }}=\right. \\
0.0665]\end{array}$ & $\begin{array}{c}7066\left[R_{\text {int }}=0.0632, R_{\text {sigma }}=\right. \\
0.0794]\end{array}$ \\
\hline Data/restraints/parameters & $6883 / 32 / 515$ & $9020 / 11 / 491$ & $7066 / 0 / 489$ \\
\hline Goodness-of-fit on $\mathrm{F}^{2}$ & 1.098 & 1.038 & 1.077 \\
\hline Final $R$ indexes $[\mathrm{I}>=2 \sigma(\mathrm{I})]$ & $\mathrm{R}_{1}=0.0795, \mathrm{wR}_{2}=0.2159$ & $\mathrm{R}_{1}=0.0407, \mathrm{wR}_{2}=0.0715$ & $\mathrm{R}_{1}=0.0528, \mathrm{wR}_{2}=0.1412$ \\
\hline Final $\mathrm{R}$ indexes [all data] & $\mathrm{R}_{1}=0.1044, \mathrm{wR}_{2}=0.2335$ & $\mathrm{R}_{1}=0.0561, \mathrm{wR}_{2}=0.0757$ & $\mathrm{R}_{1}=0.0546, \mathrm{wR}_{2}=0.1424$ \\
\hline Largest diff. peak/hole / e $\AA^{-3}$ & $2.03 /-1.74$ & $0.89 /-0.80$ & $2.23 /-1.99$ \\
\hline
\end{tabular}


Table S2. Crystallographic information for MUV-5a(Gd), MUV-5a(Ho) and MUV-5a(Tb)

\begin{tabular}{|c|c|c|c|}
\hline Identification code & MUV-5a(Gd) & MUV-5a(Ho) & MUV-5a(Tb) \\
\hline Empirical formula & $\mathrm{C}_{70} \mathrm{H}_{41.5} \mathrm{Gd}_{3} \mathrm{O}_{21.5} \mathrm{~S}_{8}$ & $\mathrm{C}_{70} \mathrm{H}_{41.5} \mathrm{Ho}_{3} \mathrm{O}_{21.5} \mathrm{~S}_{8}$ & $\mathrm{C}_{70} \mathrm{H}_{41.5} \mathrm{O}_{21.5} \mathrm{~S}_{8} \mathrm{~Tb}_{3}$ \\
\hline Formula weight & 1954.76 & 1977.80 & 1959.77 \\
\hline Temperature/K & $100(2)$ & $150(2)$ & $100(2)$ \\
\hline Crystal system & monoclinic & monoclinic & monoclinic \\
\hline Space group & $P 2 / c$ & $P 2 / c$ & $P 2 / c$ \\
\hline 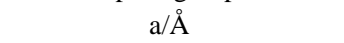 & $11.2561(4)$ & $11.1706(2)$ & $11.2108(3)$ \\
\hline $\mathrm{b} / \AA$ & $11.3522(3)$ & $11.35300(10)$ & $11.2909(4)$ \\
\hline $\mathrm{c} / \AA$ & $32.2895(8)$ & $32.3407(4)$ & $32.4084(9)$ \\
\hline$\alpha /{ }^{\circ}$ & 90 & 90 & 90 \\
\hline$\beta /{ }^{\circ}$ & $91.534(3)$ & $91.6000(10)$ & $91.719(2)$ \\
\hline$\gamma /{ }^{\circ}$ & 90 & 90 & 90 \\
\hline Volume $/ \AA^{3}$ & $4124.5(2)$ & 4099.84(10) & $4100.4(2)$ \\
\hline $\mathrm{Z}$ & 2 & 2 & 2 \\
\hline$\rho_{\text {calc }} \mathrm{g} / \mathrm{cm}^{3}$ & 1.574 & 1.602 & 1.587 \\
\hline$\mu / \mathrm{mm}^{-1}$ & 2.647 & 7.645 & 2.807 \\
\hline $\mathrm{F}(000)$ & 1907.0 & 1925.0 & 1913.0 \\
\hline Crystal size $/ \mathrm{mm}^{3}$ & $0.12 \times 0.02 \times 0.02$ & $0.22 \times 0.12 \times 0.1$ & $0.07 \times 0.01 \times 0.01$ \\
\hline Radiation & synchrotron $(\lambda=0.6889)$ & $\mathrm{CuK} \alpha(\lambda=1.54184)$ & $\operatorname{synchrotron}(\lambda=0.6889)$ \\
\hline $2 \Theta$ range for data collection ${ }^{\circ}$ & 2.446 to 49.036 & 7.918 to 152.876 & 3.496 to 49.038 \\
\hline Index ranges & $\begin{array}{c}-12 \leq \mathrm{h} \leq 13,-13 \leq \mathrm{k} \leq 13,-38 \leq 1 \leq \\
37\end{array}$ & $\leq-12 \leq \mathrm{h} \leq 13,-14 \leq \mathrm{k} \leq 13,-40 \leq 1$ & $\begin{array}{c}1 \leq-13 \leq \mathrm{h} \leq 13,-13 \leq \mathrm{k} \leq 13,-39 \leq 1 \leq \\
39\end{array}$ \\
\hline Reflections collected & 27365 & 125974 & 49332 \\
\hline Independent reflections & $\begin{array}{c}7311\left[R_{\text {int }}=0.1772, R_{\text {sigma }}=\right. \\
0.1912]\end{array}$ & $\begin{array}{c}8424\left[R_{\text {int }}=0.0478, R_{\text {sigma }}=\right. \\
0.0156]\end{array}$ & $\begin{array}{c}7505\left[R_{\text {int }}=0.0921, R_{\text {sigma }}=\right. \\
0.0581]\end{array}$ \\
\hline Data/restraints/parameters & $7311 / 0 / 484$ & $8424 / 30 / 498$ & $7505 / 30 / 493$ \\
\hline Goodness-of-fit on $\mathrm{F}^{2}$ & 1.057 & 1.104 & 1.060 \\
\hline Final $\mathrm{R}$ indexes $[\mathrm{I}>=2 \sigma(\mathrm{I})]$ & $\mathrm{R}_{1}=0.1124, \mathrm{wR}_{2}=0.2983$ & $\mathrm{R}_{1}=0.0721, \mathrm{wR}_{2}=0.1526$ & $\mathrm{R}_{1}=0.0642, \mathrm{wR}_{2}=0.1672$ \\
\hline Final $\mathrm{R}$ indexes [all data] & $\mathrm{R}_{1}=0.1230, \mathrm{wR}_{2}=0.3120$ & $\mathrm{R}_{1}=0.0724, \mathrm{wR}_{2}=0.1528$ & $\mathrm{R}_{1}=0.0717, \mathrm{wR}_{2}=0.1720$ \\
\hline Largest diff. peak/hole / e $\AA^{-3}$ & $3.07 /-1.29$ & $1.58 /-1.93$ & $2.95 /-1.57$ \\
\hline
\end{tabular}




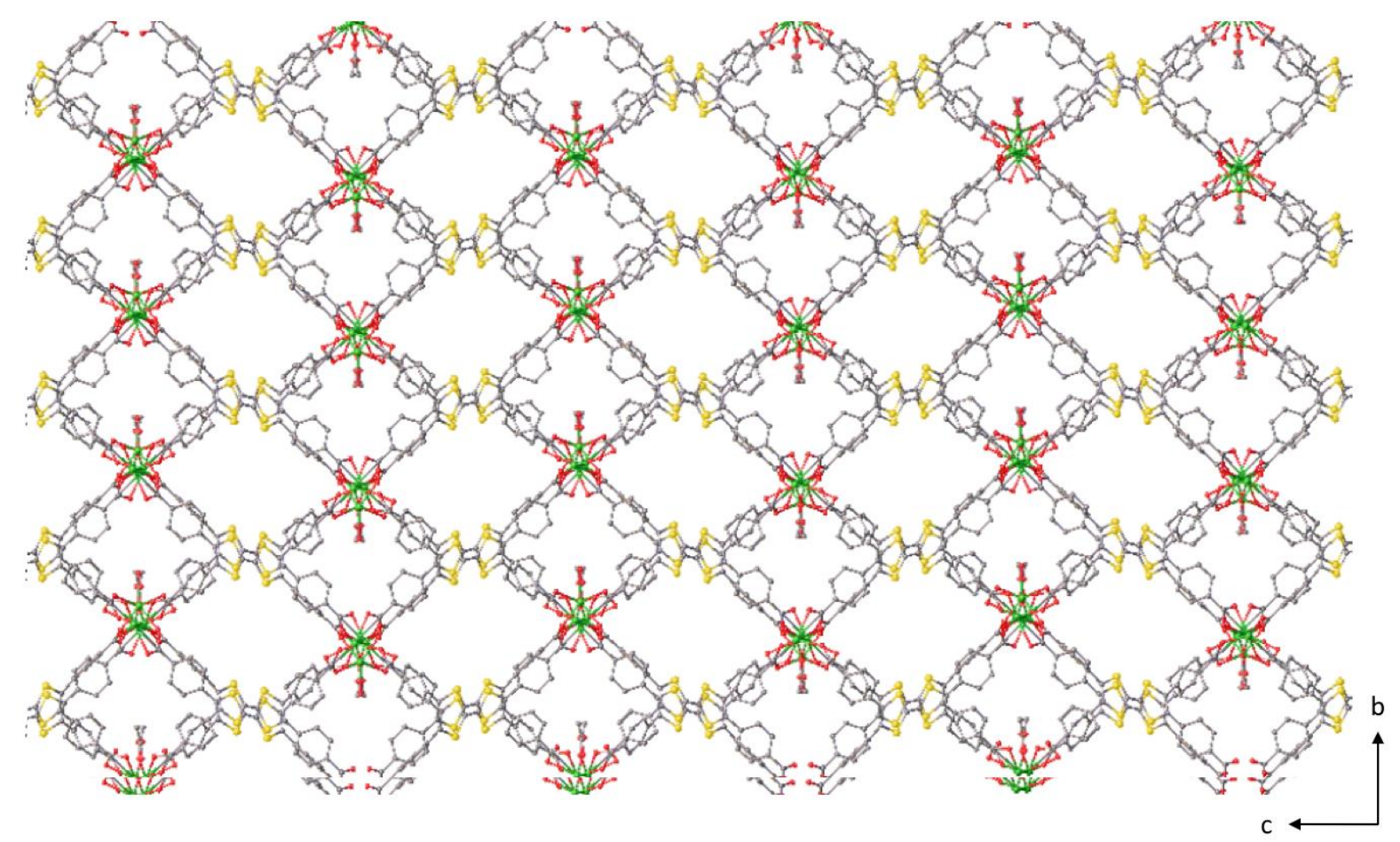

Figure S1. View of the structure of MUV-5a along the $a$-axis. Colour code: C (grey), O (red), Ln (green), S (yellow).
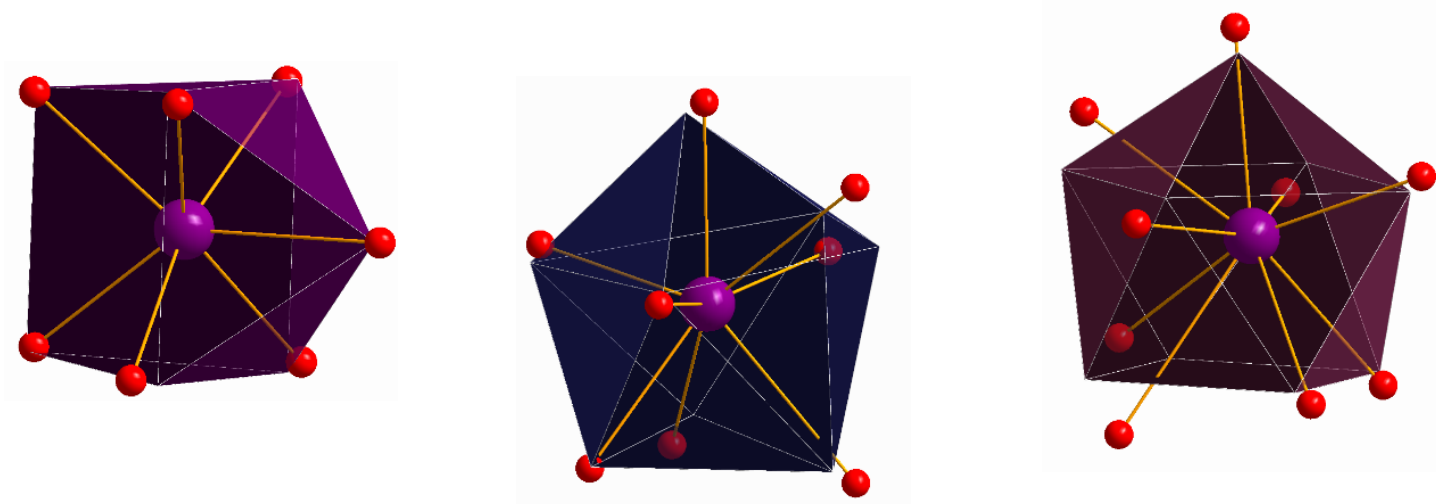

Figure S2. Coordination geometry of the Ln ions in MUV-5a: 7-coordinated Ln (left), 8-coordinated Ln (middle) and 9-coordinated Ln (right). 


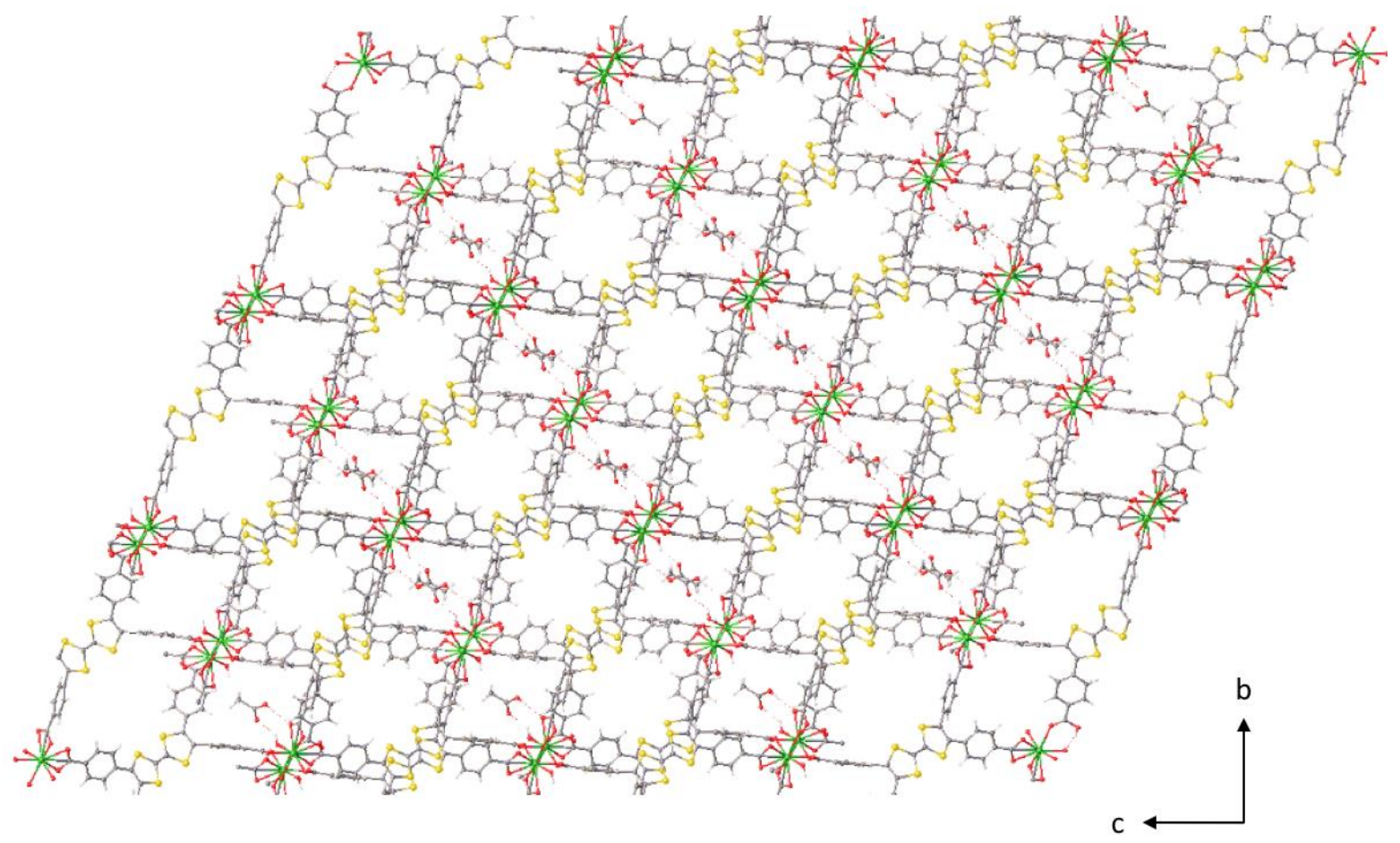

Figure S3. View of the structure of MUV-5b along the $a$-axis. Colour code: C (grey), O (red), Ln (green), S (yellow).

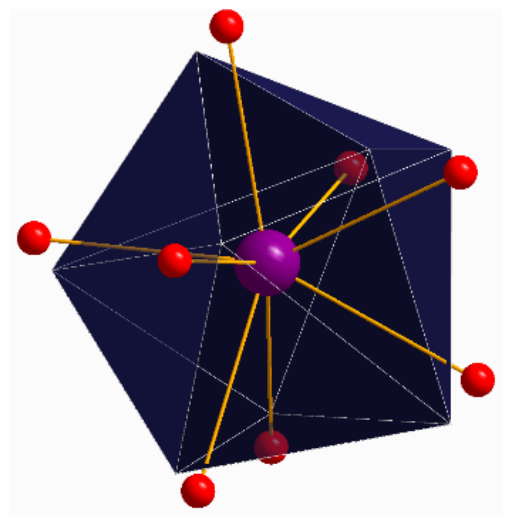

Figure S4. Coordination geometry of the 8-coordinated Ln(III) ions in MUV-5b 
MUV-5a(Dy)

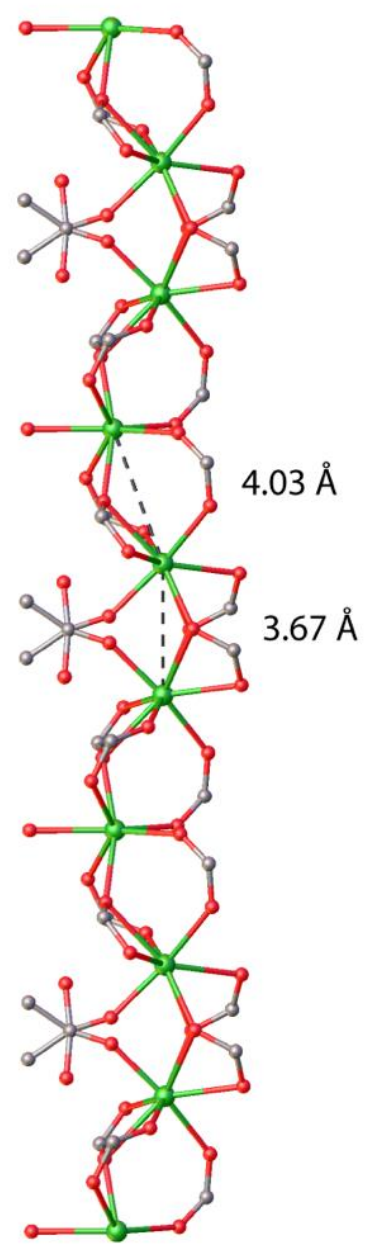

MUV-5b(Dy)
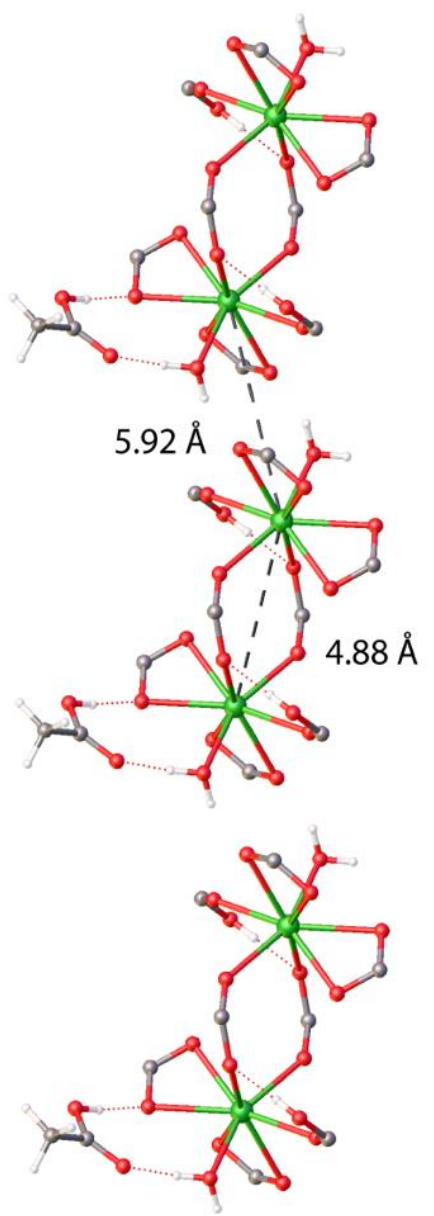

Figure S5. Comparison between the Ln-carboxylate chain found in MUV-5a(Dy) (left) and the discrete $\operatorname{Ln}_{2}$ SBU present in MUV-5b(Dy) (right). Colour code: C (grey), O (red), Ln (green), H (white). The dashed lines show the distances between Ln pairs.
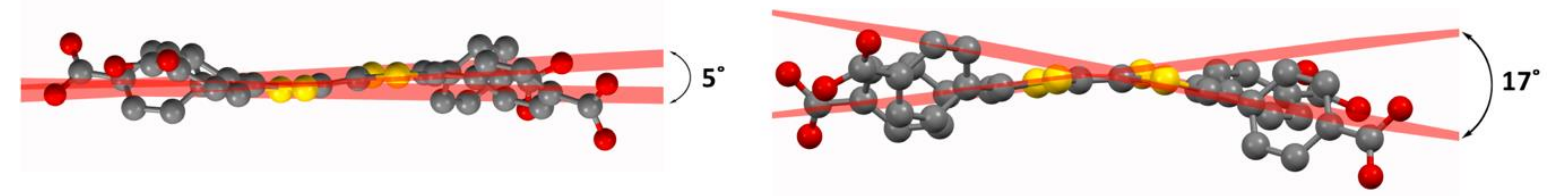

Figure S6. Calculated dihedral angles for MUV-5a(Dy) and MUV-5b(Dy). 


\section{S4. Chemical Characterization}

\section{Powder X-Ray Diffraction (PXRD)}

Powder XRD patterns were collected for polycrystalline samples using a $0.5 \mathrm{~mm}$ borosilicate capillary mounted and aligned in a PANalytical Empyrean diffractometer using copper radiation $(\mathrm{Cu} K \alpha \lambda=1.5418 \AA$ ) with an PIXcel detector, operating at $40 \mathrm{~mA}$ and $45 \mathrm{kV}$. Profiles for refinement were collected by using a Soller Slit of $0.02^{\circ}$ and a divergence slit of $1 / 4$ at room temperature in the angular range $3^{\circ}<2 \theta<40^{\circ}$ with a step size of $0.013^{\circ}$. LeBail refinements were carried out with the FULLPROF software package.

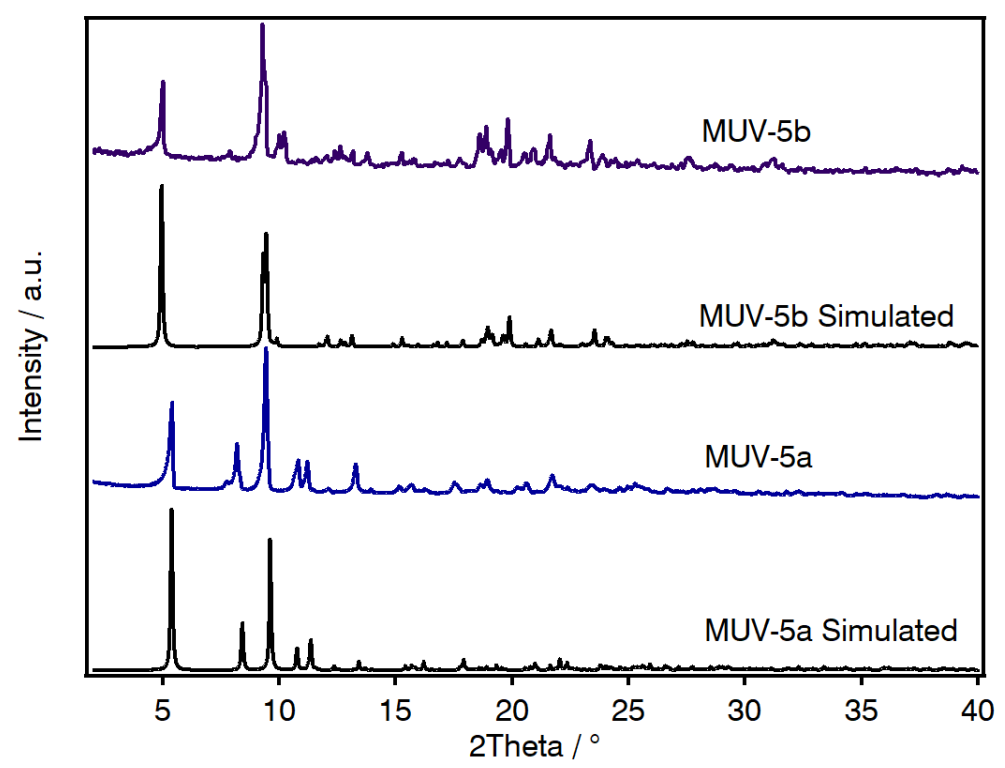

Figure S7. PXRD of simulated and experimental MUV-5a(Dy) and MUV-5b(Dy).

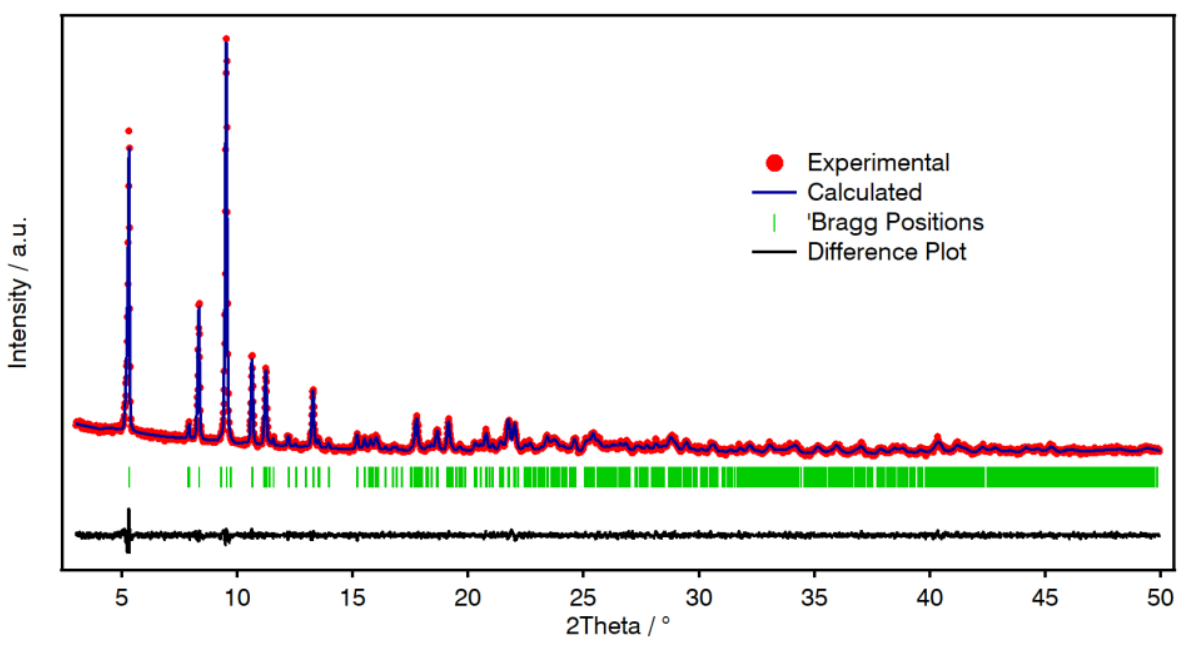

Figure S8. Experimental (red dots), calculated (blue line), difference plot $\left[\left(I_{\mathrm{obs}}-I_{\mathrm{calc}}\right)\right]$ (black line) and Bragg positions (green ticks) for the LeBail refinement of experimental diffraction data of MUV-5a(Dy) collected at room temperature by using single-crystal data from MUV-5a(Dy) as starting parameters 
Table S3. Summary of the parameters obtained from LeBail refinement. Note that the differences observed can be attributed to the different experimental temperatures.

\begin{tabular}{cccccccccc} 
Compound & $\begin{array}{c}a \\
{[\AA]}\end{array}$ & $\begin{array}{c}b \\
{[\AA]}\end{array}$ & $\begin{array}{c}c \\
{[\AA]}\end{array}$ & $\begin{array}{c}\beta \\
{\left[{ }^{\circ}\right]}\end{array}$ & $\begin{array}{c}V \\
{\left[\AA^{3}\right]}\end{array}$ & $\begin{array}{c}\mathrm{R}_{\mathrm{e}} \\
{[\%]}\end{array}$ & $\begin{array}{c}\mathrm{R}_{\mathrm{p}} \\
{[\%]}\end{array}$ & $\begin{array}{c}\mathrm{R}_{\mathrm{wp}} \\
{[\%]}\end{array}$ & gof \\
\hline MUV-5a(Dy) & 11.247 & 11.168 & 33.254 & 92.67 & 4172 & 4.92 & 4.36 & 5.58 & 1.1 \\
\hline $\begin{array}{l}\text { MUV-5a(Dy) } \\
\text { Single crystal }\end{array}$ & 11.188 & 11.078 & 32.841 & 91.771 & 4068 & - & - & - & -
\end{tabular}

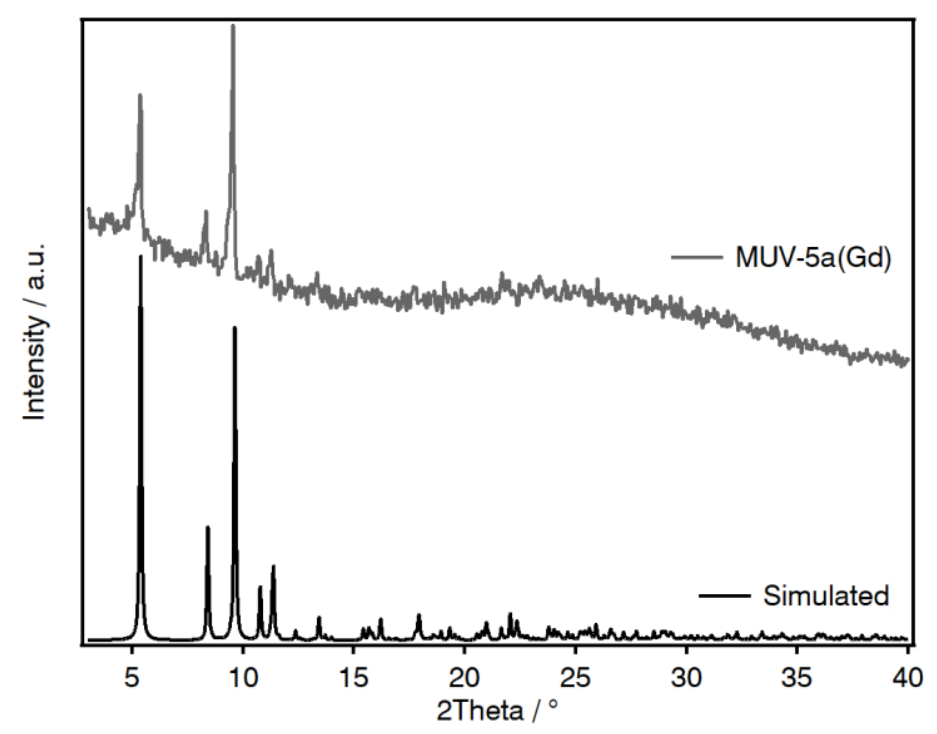

Figure S9. Experimental (grey) and simulated (black) PXRD of MUV-5a(Gd).

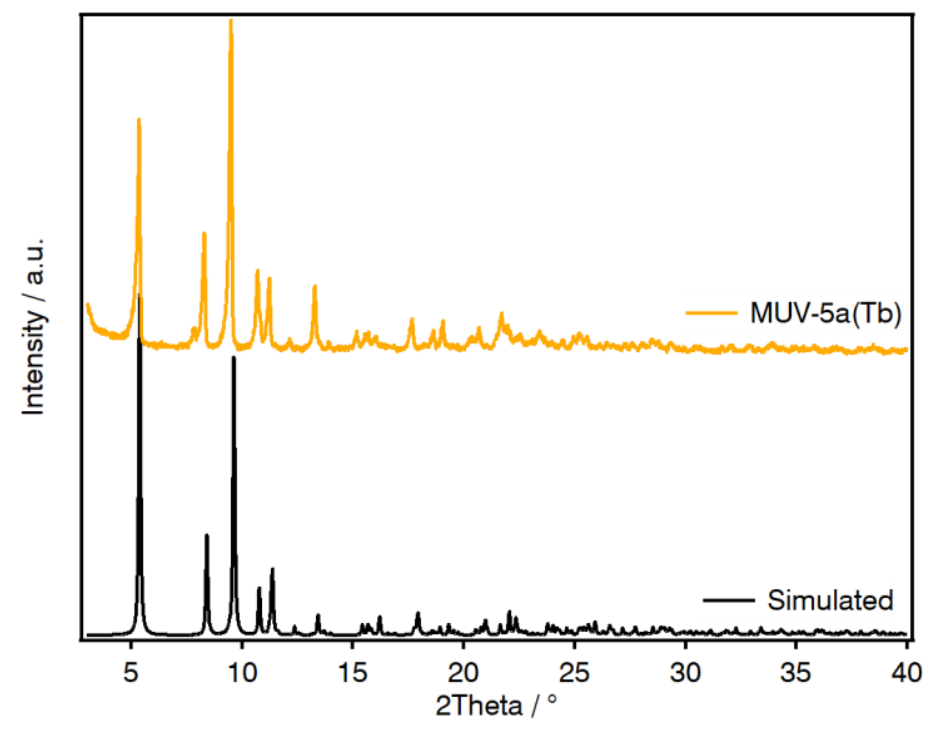

Figure S10. Experimental (yellow) and simulated (black) PXRD of MUV-5a(Tb). 


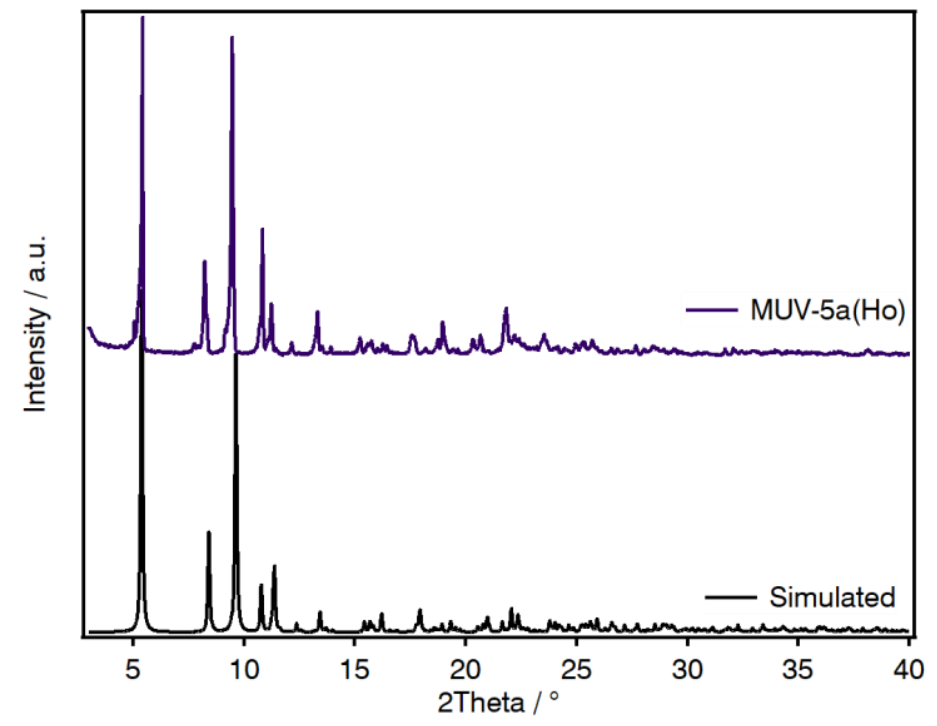

Figure S11. Experimental (purple) and simulated (black) PXRD of MUV-5a(Ho).

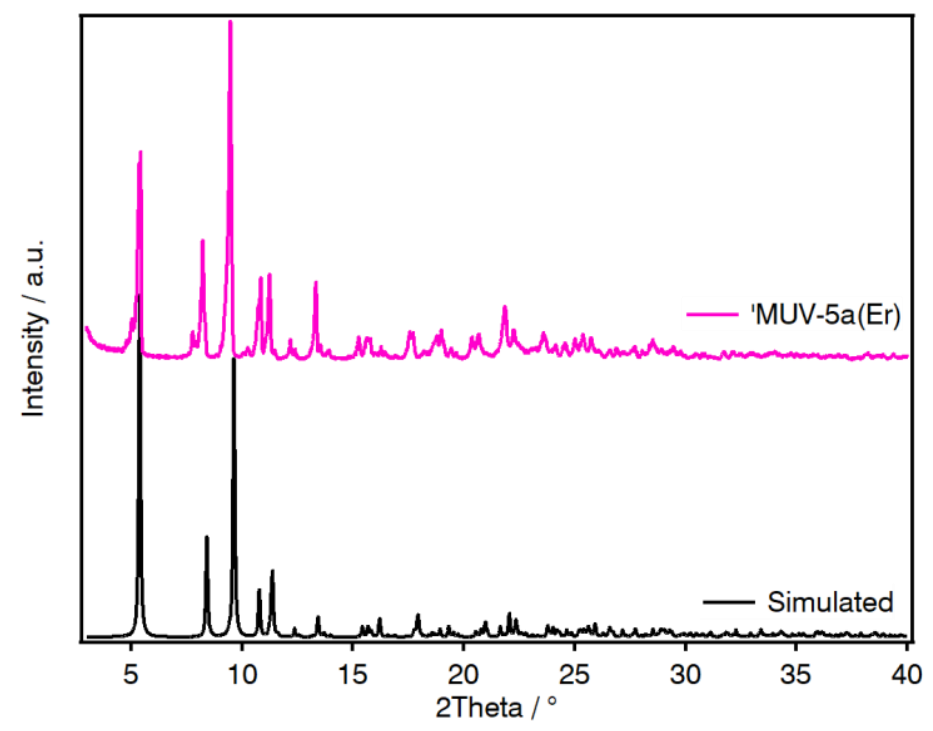

Figure S12. Experimental (pink) and simulated (black) PXRD of MUV-5a(Er). 


\section{Thermogravimetric Analysis (TG-SDTA)}

TGA-SDTA curves were recorded at a ramp rate of $5{ }^{\circ} \mathrm{C} / \mathrm{min}$ on a Mettler Toledo TGA/SDTA $851 \mathrm{e} / \mathrm{SF} / 1100$ apparatus between 25 and $700{ }^{\circ} \mathrm{C}$ under synthetic air.

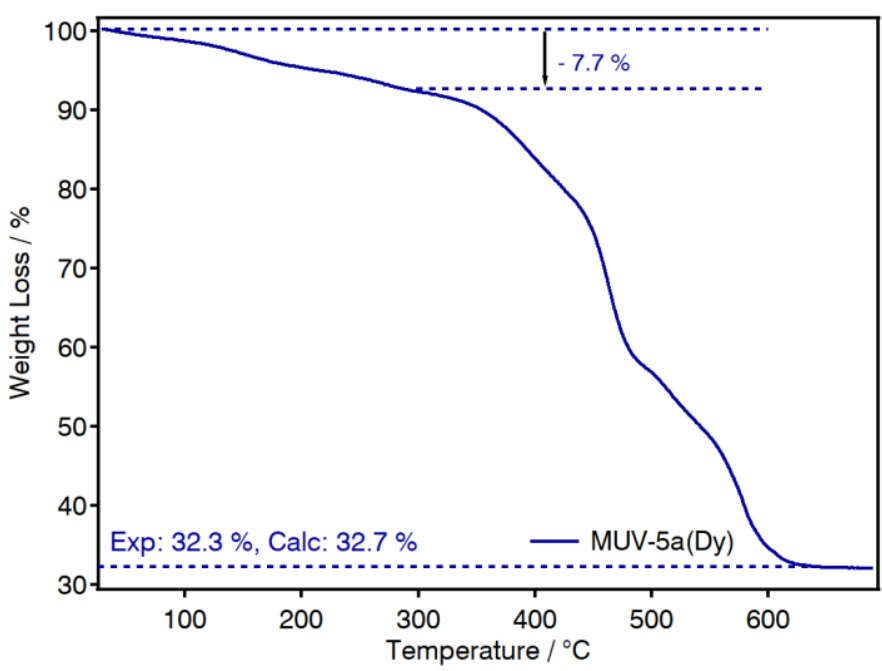

Figure S13. Thermogravimetric analysis of MUV-5a(Dy) in air. The first weight loss corresponds to the departure of DMF molecules $\left(-2 \cdot\left(\mathrm{CH}_{3}\right)_{2} \mathrm{NCHO}\right.$; Exp.: $7.7 \%$; Calcd.: $\left.7.5 \%\right)$.

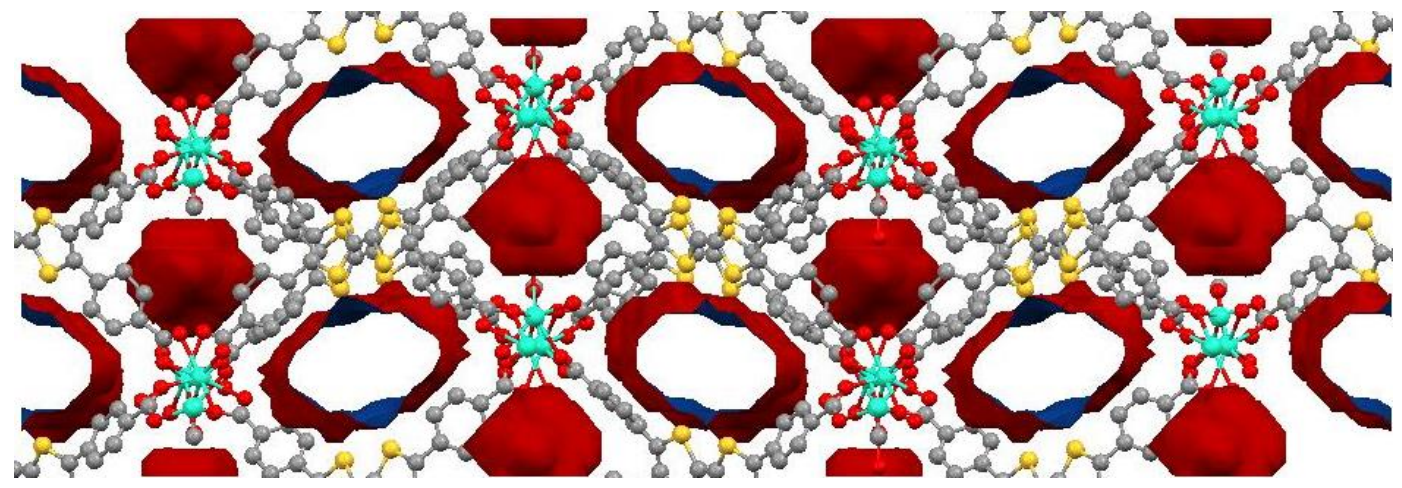

Figure S14. Representation of the void volume of MUV-5a as calculated by Mercury. 


\section{Gas adsorption/desorption isotherms}

Gas adsorption measurements were recorded ex situ on MUV-5a solids on a Micromeritics 3Flex apparatus. Samples were degassed overnight at $150^{\circ} \mathrm{C}$ and $10^{-6}$ Torr prior to analysis. High-pressure gas sorption studies (up to 12 bar) were performed in a gravimetric sorption analyser IGA-100 (Hiden Isochema). The sample was degassed 2 hours at $150{ }^{\circ} \mathrm{C}$ under vacuum. $\mathrm{CO}_{2}$ adsorption isotherms were acquired at 10, 25, 40 and $60{ }^{\circ} \mathrm{C}$.
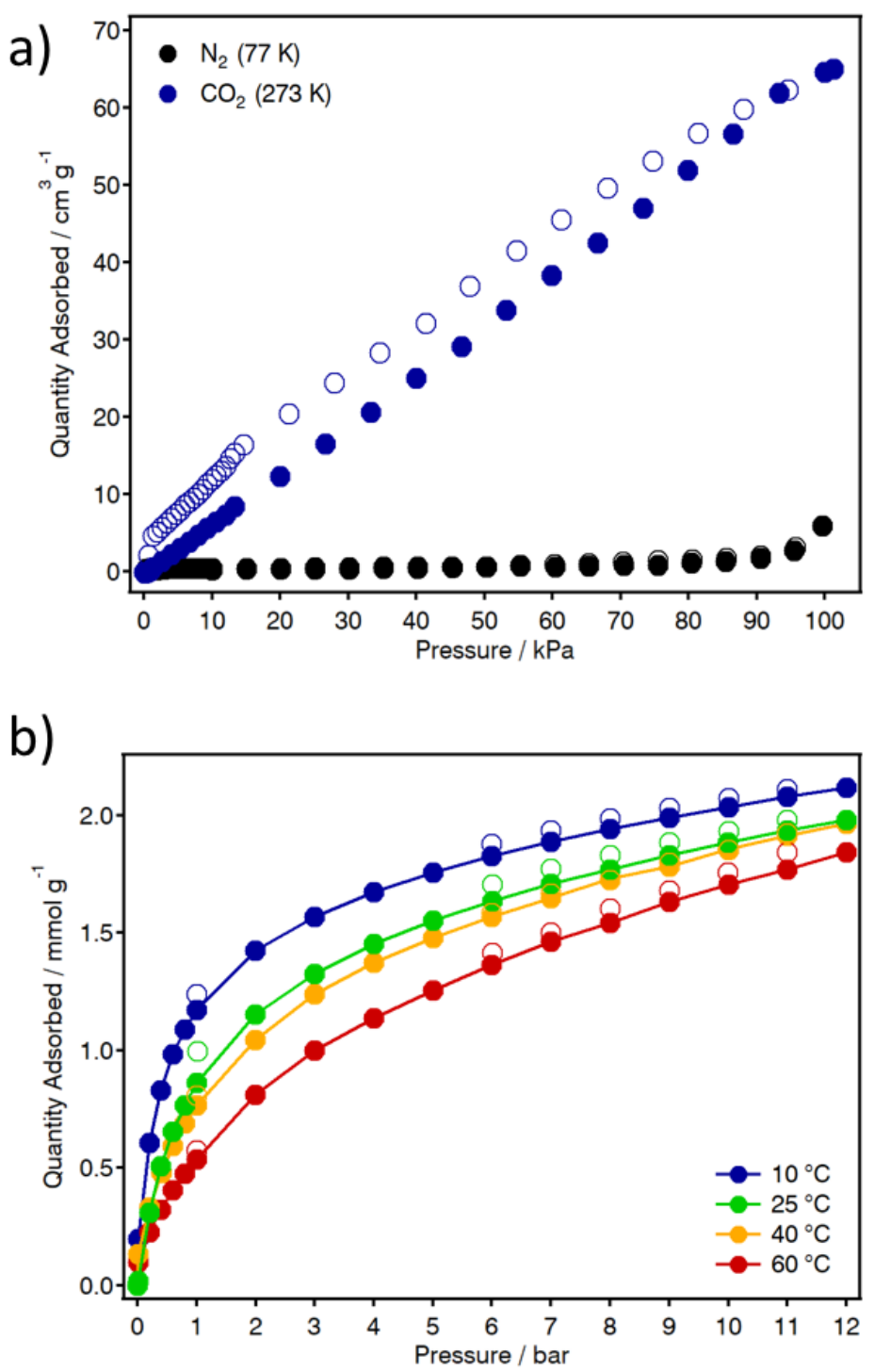

Figure S15. a) $\mathrm{N}_{2}$ (black) and $\mathrm{CO}_{2}$ (blue) volumetric adsorption-desorption isotherms of MUV-5a(Dy) performed at 77 and $273 \mathrm{~K}$, respectively. Filled and open symbols represent the adsorption and the desorption branch, respectively. b) Gravimetric adsorption (closed symbols) and desorption (open symbols) isotherms of $\mathrm{CO}_{2}$ at different temperatures in MUV-5a(Dy). 


\section{Electron Paramagnetic Resonance (EPR)}

EPR measurements were performed on approximately $3 \mathrm{mg}$ of solid. EPR data was recorded in a Bruker ELEXYS E580 spectrometer under X-band irradiation $(\sim 9.3 \mathrm{GHz})$ at room temperature.

a)

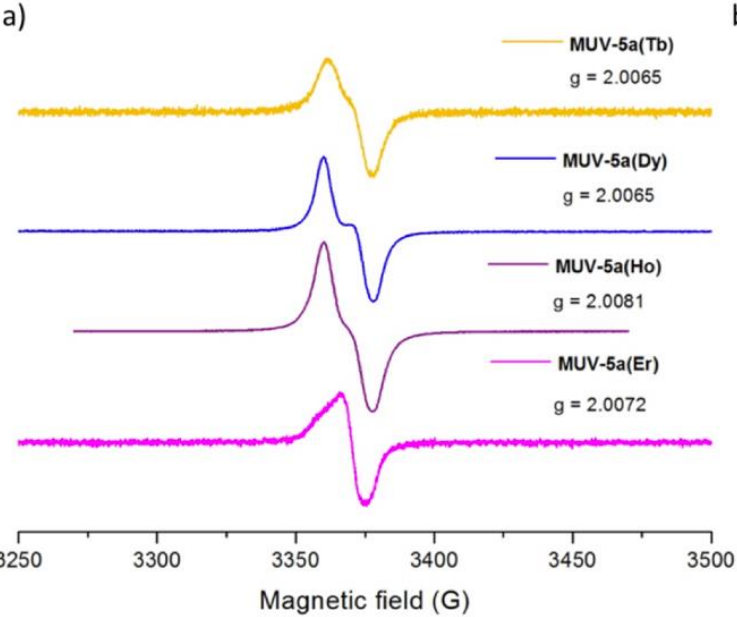

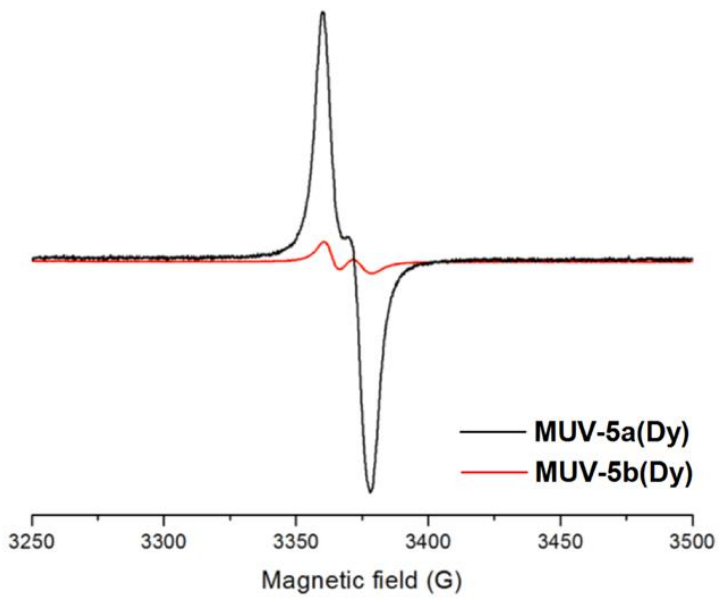

Figure S16. a) EPR spectra of MUV-5a(Ln) recorded at room temperature. b) Comparison of EPR intensities for MUV-5a(Dy) and MUV-5b(Dy) at room temperature.

\section{Scanning Electron Microscope (SEM)}

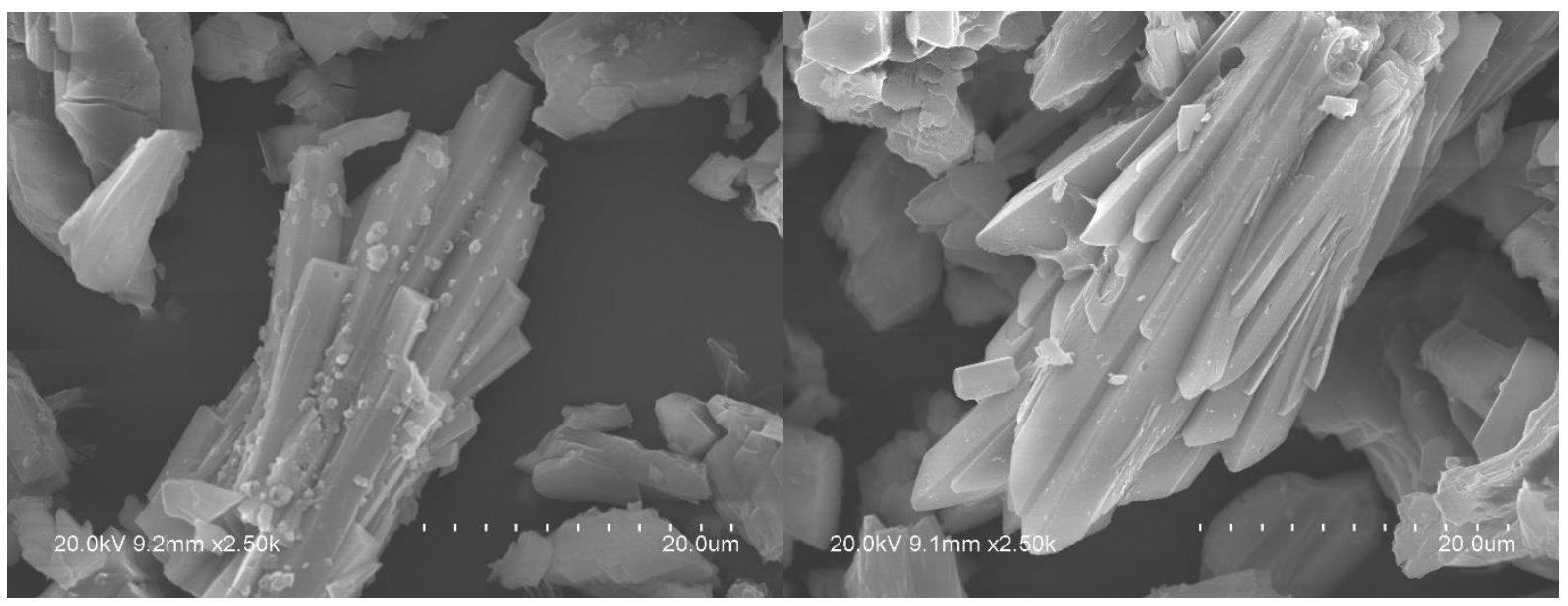

Figure S17. Scanning Electron Microscopy (SEM) images of MUV-5a(Dy). 


\section{S5. Electrical Measurements}

Powders of MUV-5 were pressed to form pellets ( $\mathrm{P} \approx 5$ US tons), cut with rectangular shapes and contacted with platinum wires (Goodfellow, 99.99\%, $25 \mu \mathrm{m}$ of diameter) and silver conductive paint (RS 123-9911) in a 4 probe configuration (Figures S18-S29). The geometrical factors (width, length and thickness) were measured using an optical microscope (width and length were determined from the top view, Figures S18-26a, and the thickness from the lateral one, Figures S18-29b). I-V curves were measured with a Keithley 2450 sourcemeter, applying a current bias through the external leads and measuring the voltage drop by the internal ones. The conductivity $(\sigma=$ $\frac{1}{R} \cdot \frac{l}{w \cdot t}$, where $\mathrm{R}$ is the resistance, 1 is the length between the leads, $\mathrm{w}$ is the width of the sample and $\mathrm{t}$ its thickness) was obtained taking into account the geometrical factors and the resistance obtained by fitting the ohmic regime of the I-V curves (Figures S18-29c).

Typical samples and an I-V curve at room temperature is shown in Figures S18 - S29. The values obtained for the different MUV-5 samples are summarized in the Table S4.

Table S4. Geometrical factors (length, 1, width, w, and thickness, t), resistance obtained by the linear fit of the ohmic regime of the I-V curves and conductivity at $300 \mathrm{~K}$ for the different MUV-5 systems.

\begin{tabular}{cccccc}
\hline MUV-5a & $\mathbf{I}(\boldsymbol{\mu \mathbf { m }})$ & $\mathbf{w}(\boldsymbol{\mu \mathbf { m }})$ & $\mathbf{t}(\boldsymbol{\mu \mathbf { m }})$ & $\mathbf{R}(\boldsymbol{\Omega}) \mathbf{a t} \mathbf{3 0 0} \mathbf{K}$ & $\boldsymbol{\sigma}(\mathbf{S} / \mathbf{c m})$ at 300 K \\
\hline \hline Dy\#1 & 130 & 370 & 90 & $(8.35 \pm 0.016) \cdot 10^{7}$ & $4.68 \cdot 10^{-7}$ \\
Dy\#2 & 190 & 335 & 210 & $(1.41 \pm 0.03) \cdot 10^{8}$ & $1.92 \cdot 10^{-7}$ \\
Gd\#1 & 185 & 430 & 130 & $(1.032 \pm 0.16) \cdot 10^{8}$ & $3.21 \cdot 10^{-7}$ \\
Gd\#2 & 320 & 370 & 200 & $(5.49 \pm 0.05) \cdot 10^{8}$ & $7.87 \cdot 10^{-8}$ \\
Tb\#1 & 245 & 320 & 105 & $(9.34 \pm 0.04) \cdot 10^{6}$ & $7.81 \cdot 10^{-6}$ \\
Tb\#2 & 340 & 680 & 85 & $(2.75 \pm 0.02) \cdot 10^{6}$ & $2.13 \cdot 10^{-5}$ \\
Ho\#1 & 290 & 635 & 405 & $(3.604 \pm 0.005) \cdot 10^{6}$ & $3.12 \cdot 10^{-6}$ \\
Ho\#2 & 240 & 615 & 145 & $(2.602 \pm 0.002) \cdot 10^{6}$ & $1.03 \cdot 10^{-5}$ \\
Er\#1 & 375 & 580 & 160 & $(3.347 \pm 0.016) \cdot 10^{6}$ & $1.21 \cdot 10^{-5}$ \\
Er\#2 & 270 & 660 & 215 & $(7.0164 \pm 0.0008) \cdot 10^{6}$ & $2.71 \cdot 10^{-6}$ \\
\hline \hline MUV-5b & $\mathbf{I}(\boldsymbol{\mu m})$ & $\mathbf{w}(\boldsymbol{\mu m})$ & $\mathbf{t}(\boldsymbol{\mu m})$ & $\mathbf{R}(\boldsymbol{\Omega}) \mathbf{a t ~ 3 0 0 ~ K}$ & $\boldsymbol{\sigma}(\mathbf{S} / \mathbf{c m}) \mathbf{a t} \mathbf{3 0 0} \mathbf{K}$ \\
\hline \hline Dy\#1 & 370 & 590 & 150 & $(1.214 \pm 0.004) \cdot 10^{9}$ & $3.43 \cdot 10^{-8}$ \\
Dy\#1 & 230 & 400 & 170 & $(1.0466 \pm 0.0019) \cdot 10^{9}$ & $3.23 \cdot 10^{-8}$ \\
\hline \hline
\end{tabular}




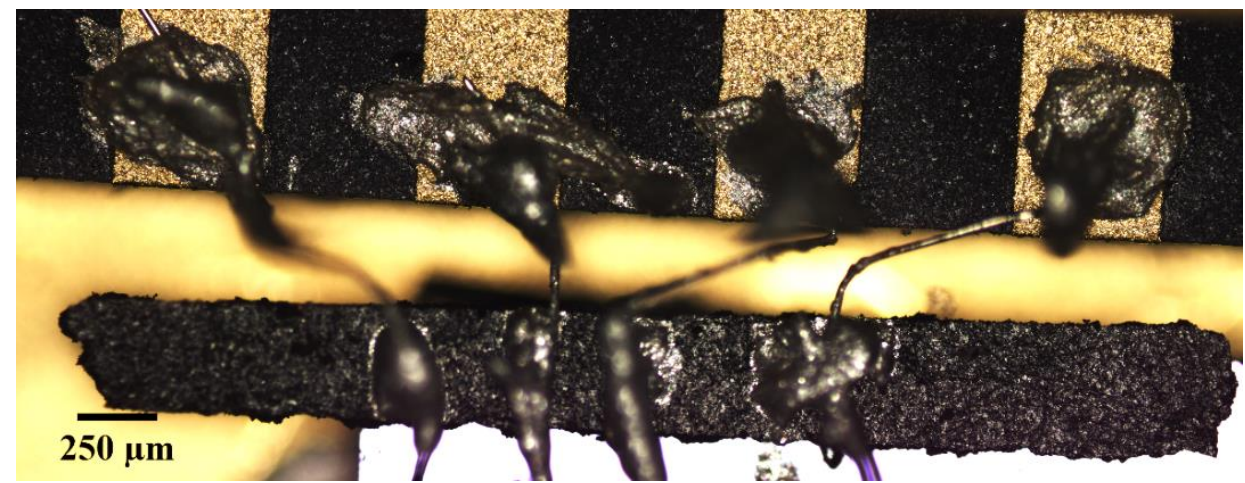

b

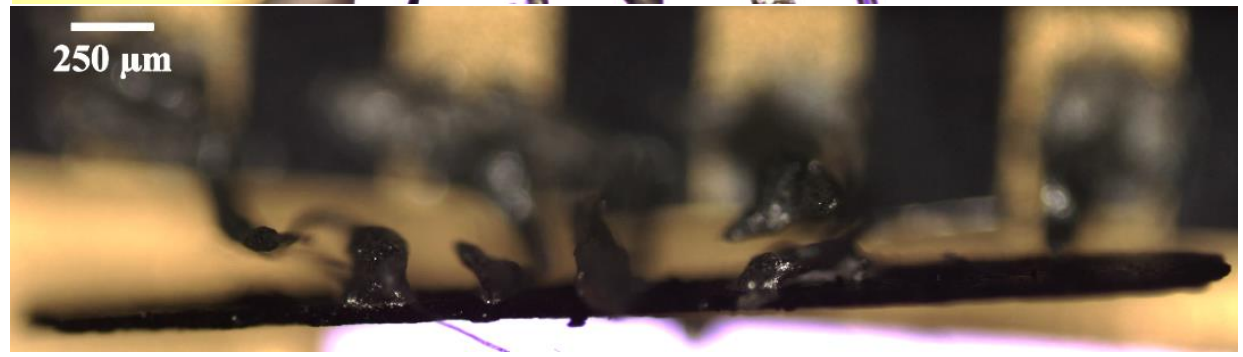

c

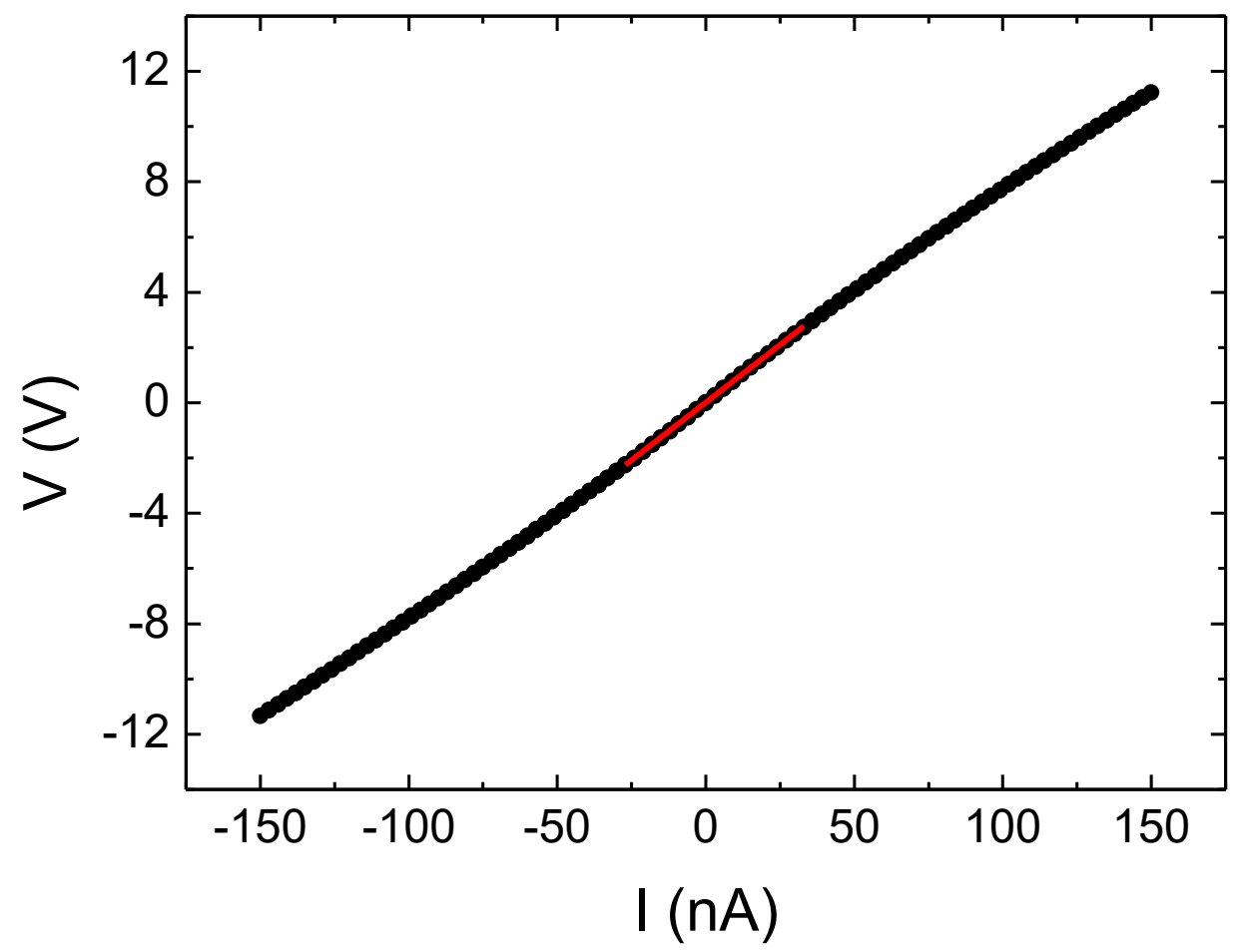

Figure S18. Device and electrical response of MUV-5a(Dy)\#1. a) Top view. b) Lateral view. c) I-V curve at 300 $\mathrm{K}$ with the corresponding linear fit (red) of the ohmic regime. The fit gives as a result a slope of $(8.35 \pm 0.016) \cdot 10^{7}$ $\Omega$ and a y-intercept value of $(0.012 \pm 0.003) \mathrm{V}$, with $\mathrm{R}^{2}=0.99992$. 
a

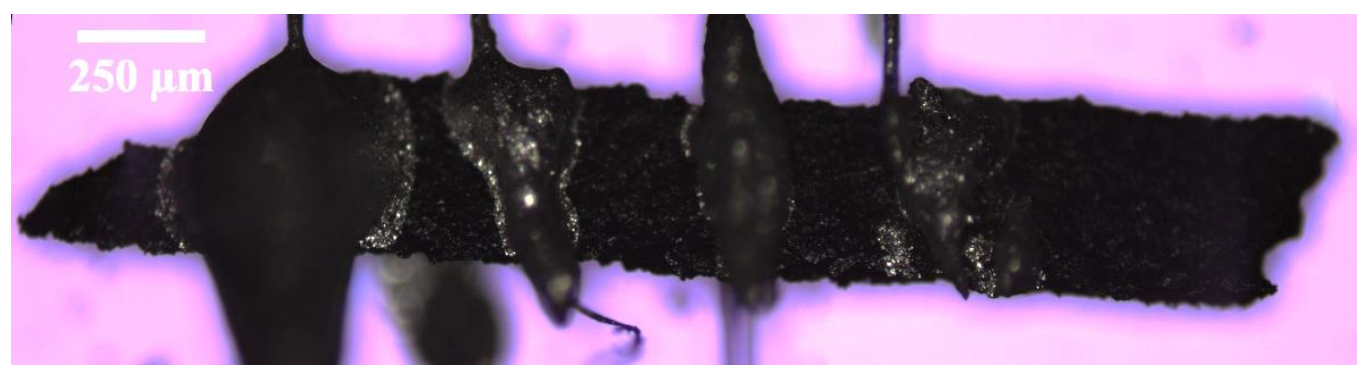

b

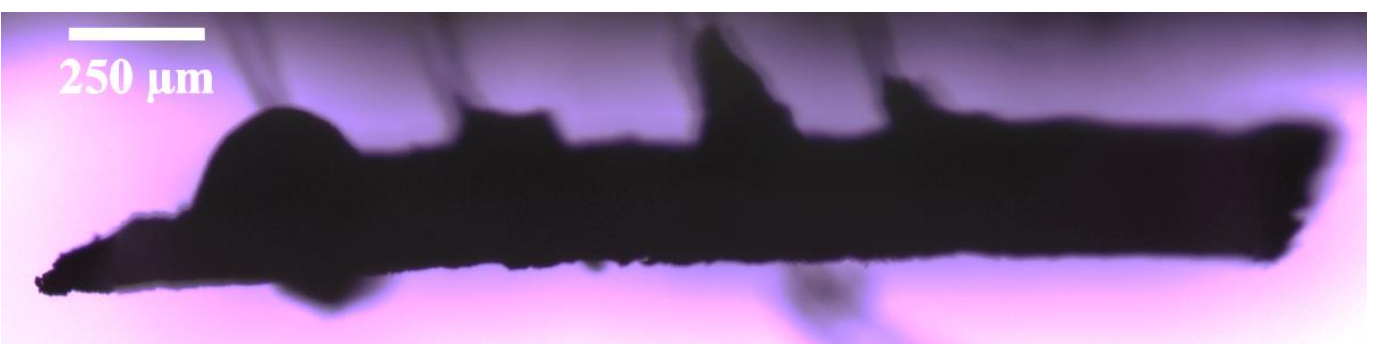

c

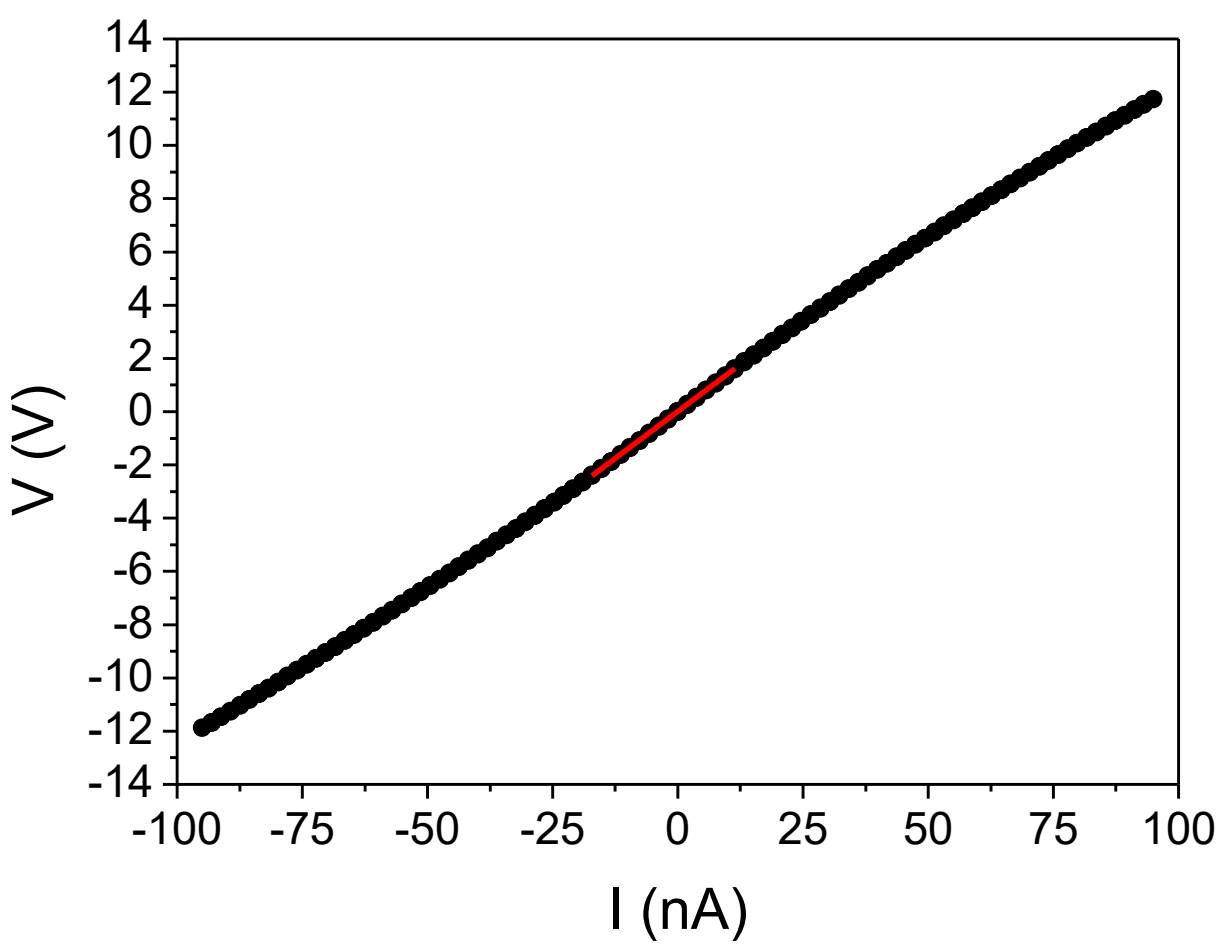

Figure S19. Device and electrical response of MUV-5a(Dy)\#2. a) Top view. b) Lateral view. c) I-V curve at 300 $\mathrm{K}$ with the corresponding linear fit (red) of the ohmic regime. The fit gives as a result a slope of $(1.41 \pm 0.03)$. $10^{8} \Omega$ and a y-intercept value of $(0.011 \pm 0.003) \mathrm{V}$, with $\mathrm{R}^{2}=0.99994$. 


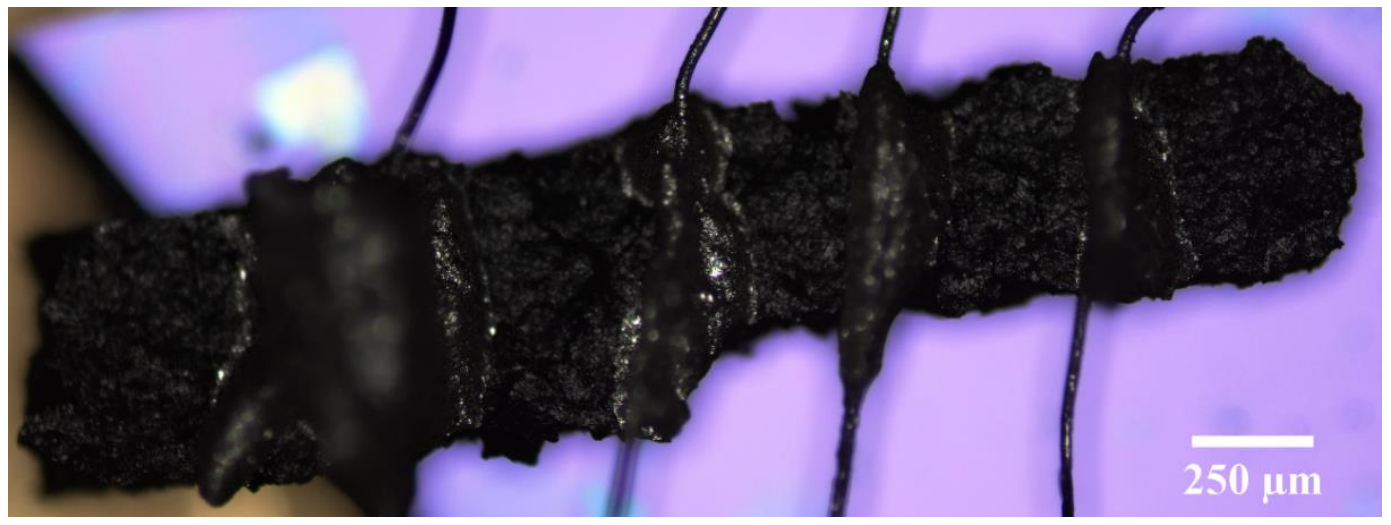

b

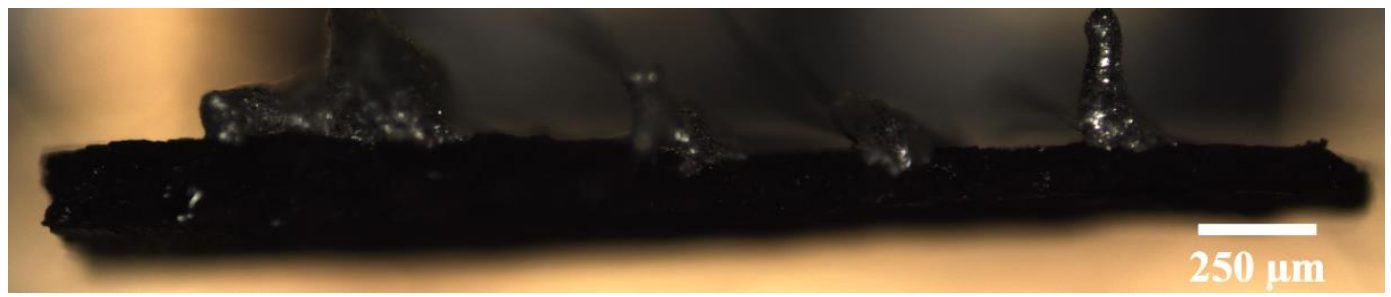

c

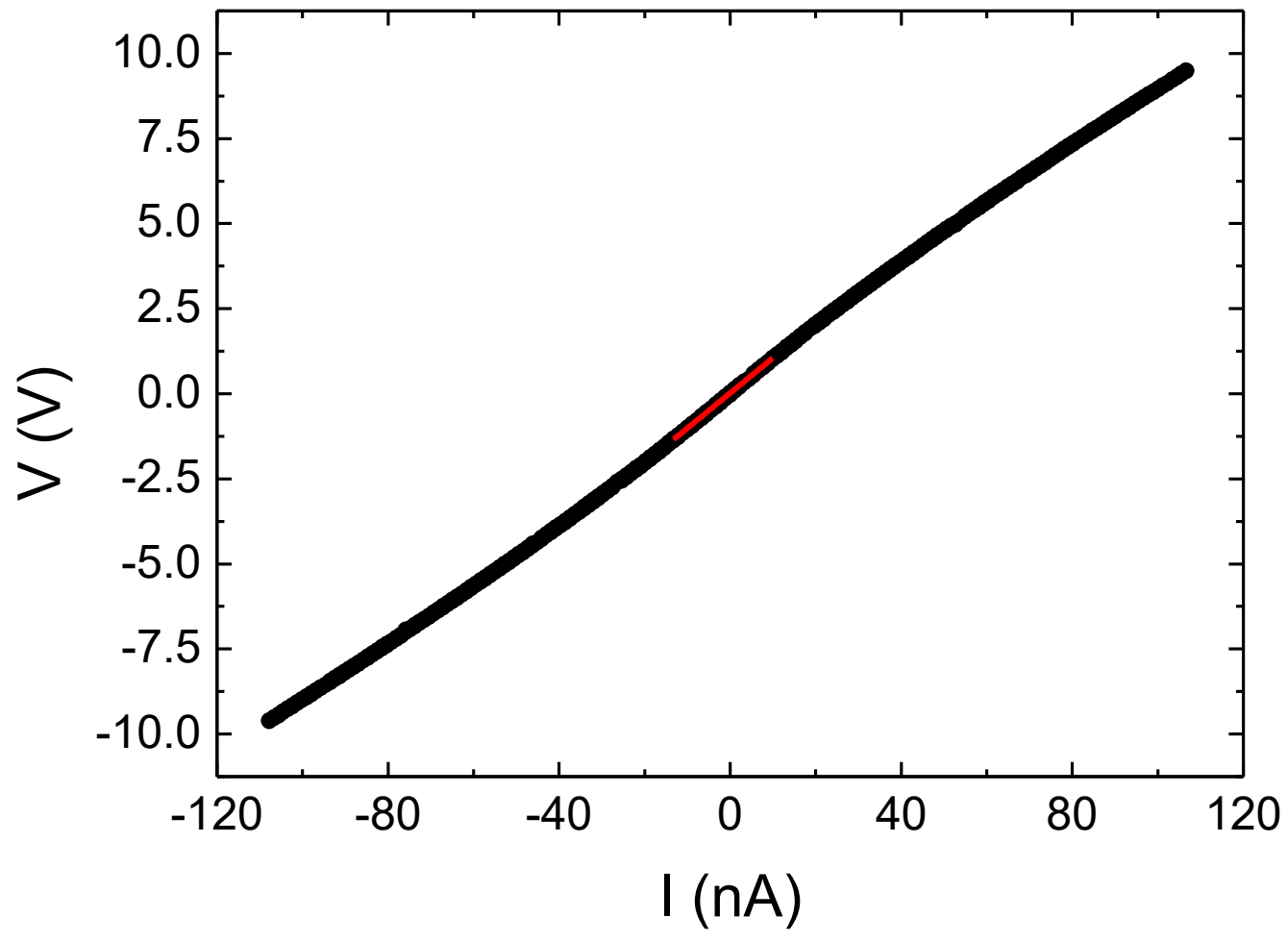

Figure S20. Device and electrical response of MUV-5a(Gd)\#1. a) Top view. b) Lateral view. c) I-V curve at 300 $\mathrm{K}$ with the corresponding linear fit (red) of the ohmic regime. The fit gives as a result a slope of $(1.032 \pm 0.16) \cdot 10^{8}$ $\Omega$ and a y-intercept value of $(0.0216 \pm 0.0012) \mathrm{V}$, with $\mathrm{R}^{2}=0.99995$. 


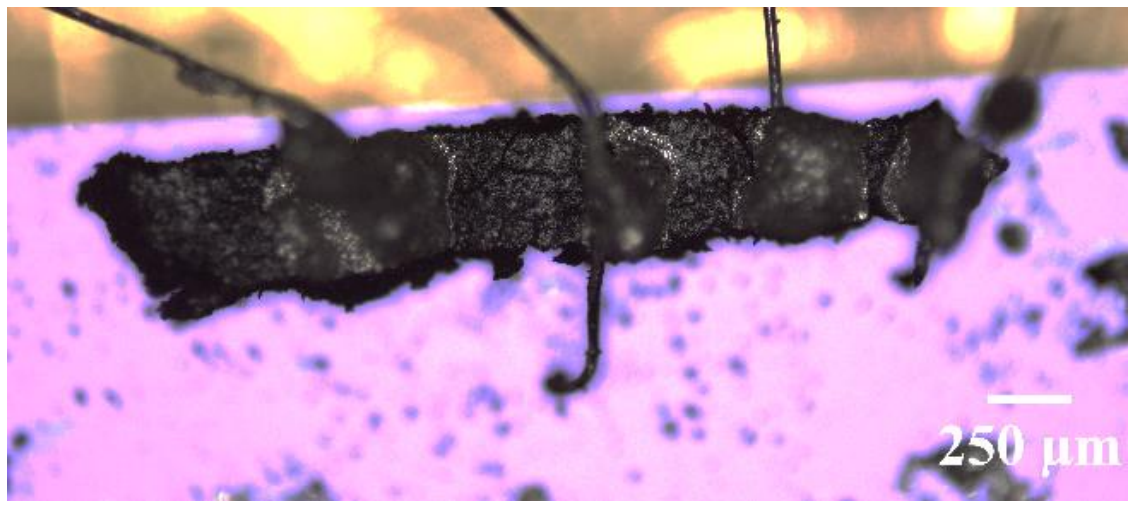

b

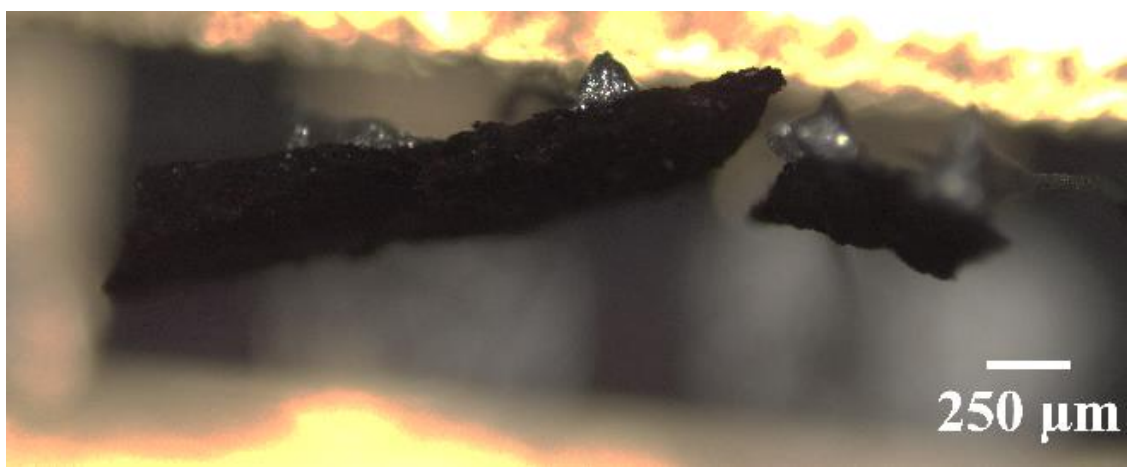

c

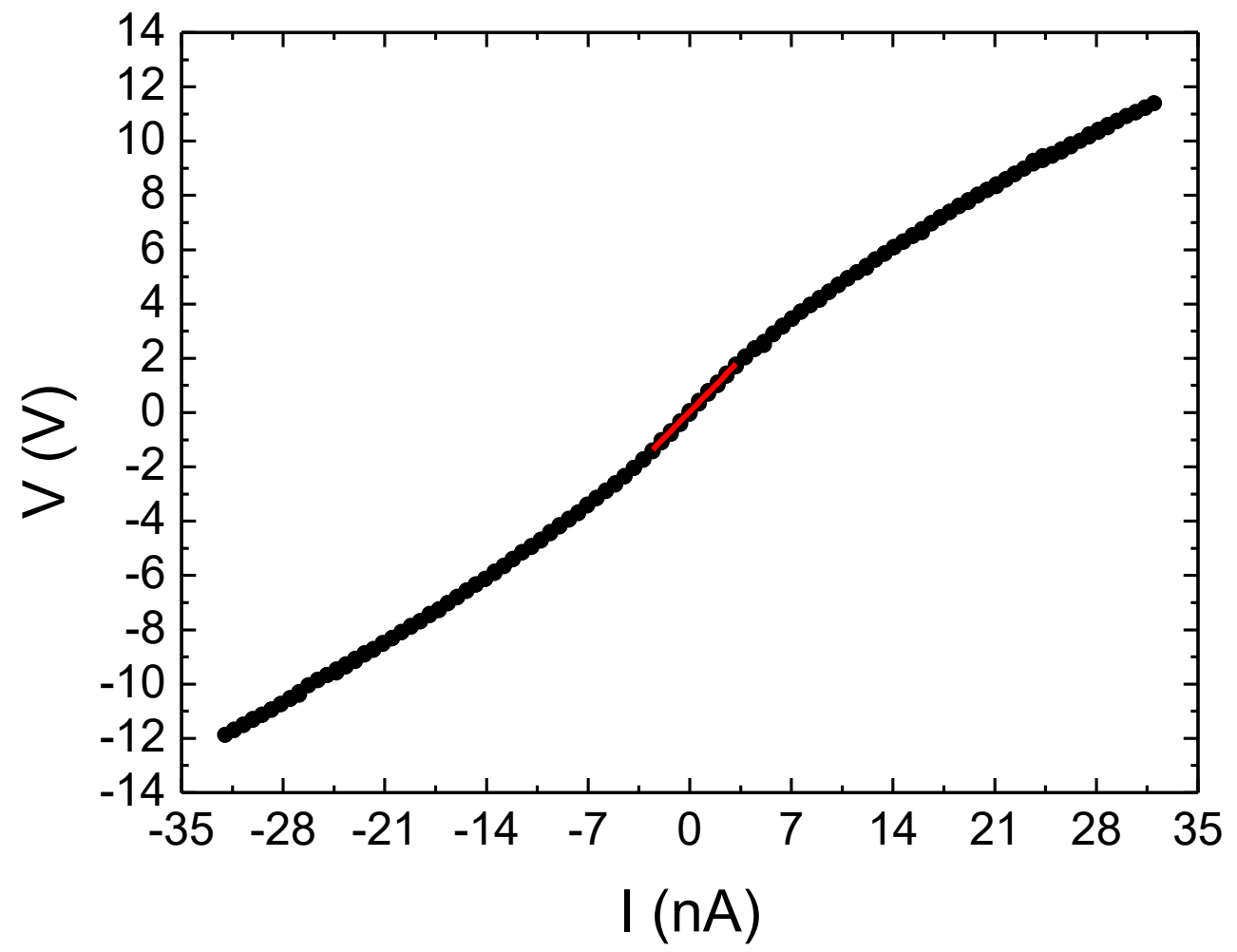

Figure S21. Device and electrical response of MUV-5a(Gd)\#2. a) Top view. b) Lateral view. c) I-V curve at 300 $\mathrm{K}$ with the corresponding linear fit (red) of the ohmic regime. The fit gives as a result a slope of $(5.49 \pm 0.05) \cdot 10^{8}$ $\Omega$ and a y-intercept value of $(0.034 \pm 0.008) \mathrm{V}$, with $\mathrm{R}^{2}=0.9994$. 


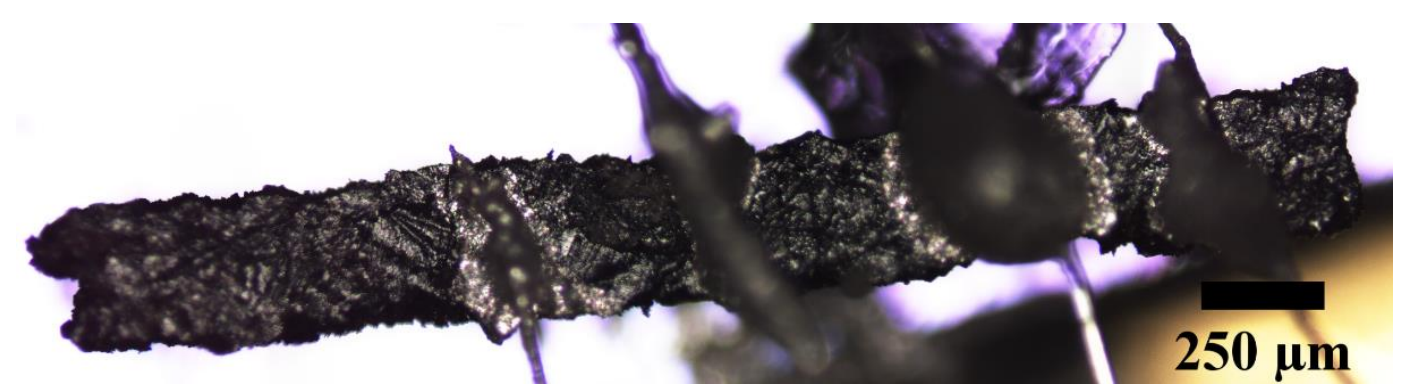

b

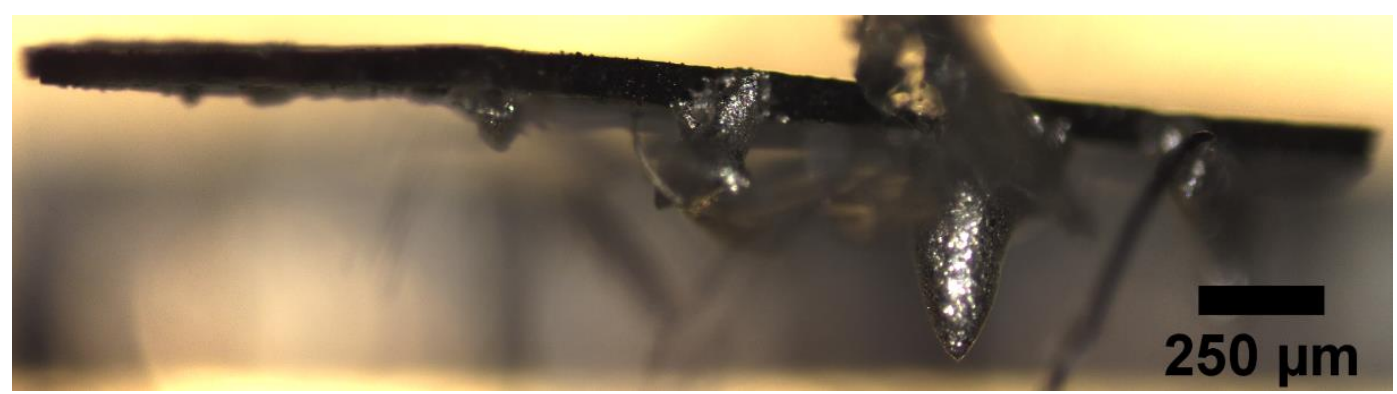

c

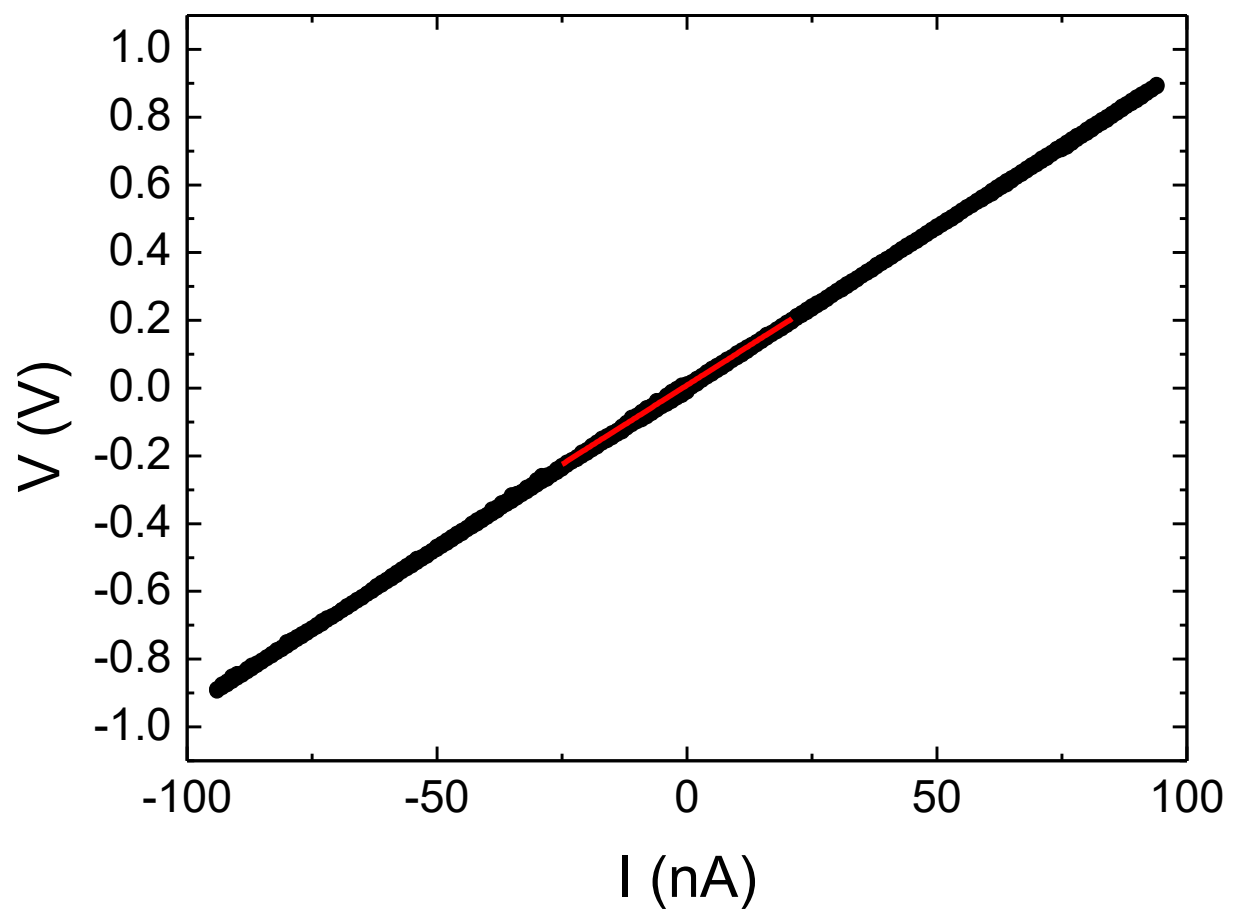

Figure S22. Device and electrical response of MUV-5a(Tb)\#1. a) Top view. b) Lateral view. c) I-V curve at 300 $\mathrm{K}$ with the corresponding linear fit (red) of the ohmic regime. The fit gives as a result a slope of $(9.34 \pm 0.04) \cdot 10^{6}$ $\Omega$ and a y-intercept value of $(0.0086 \pm 0.0006) \mathrm{V}$, with $\mathrm{R}^{2}=0.99902$. 
a

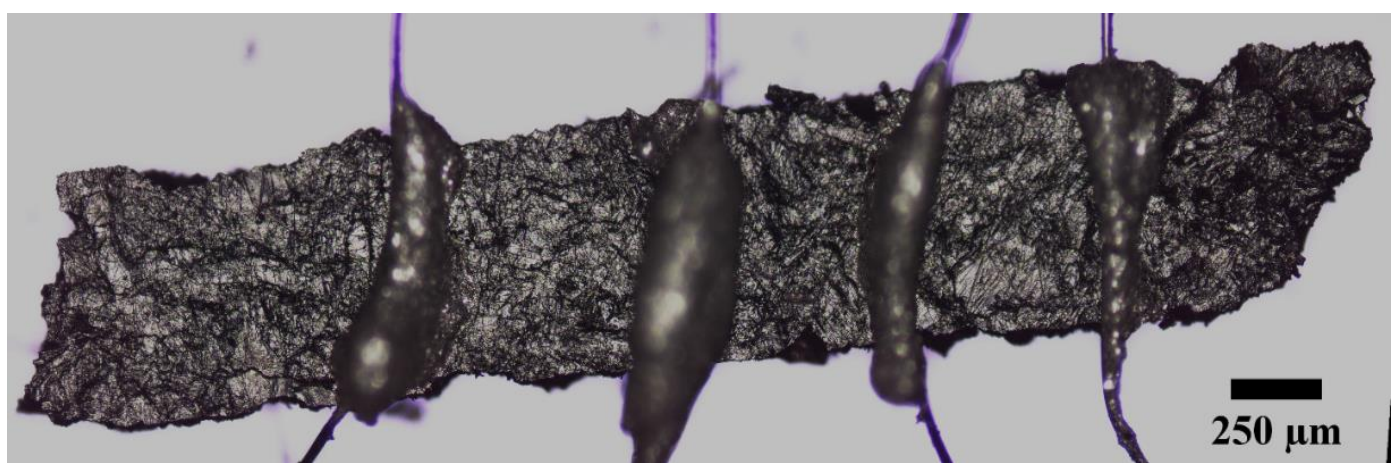

b

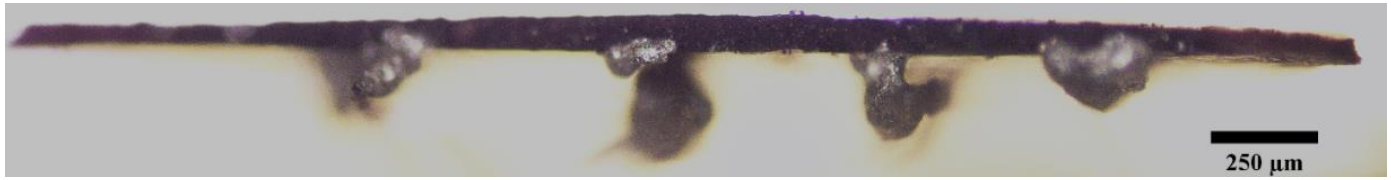

c

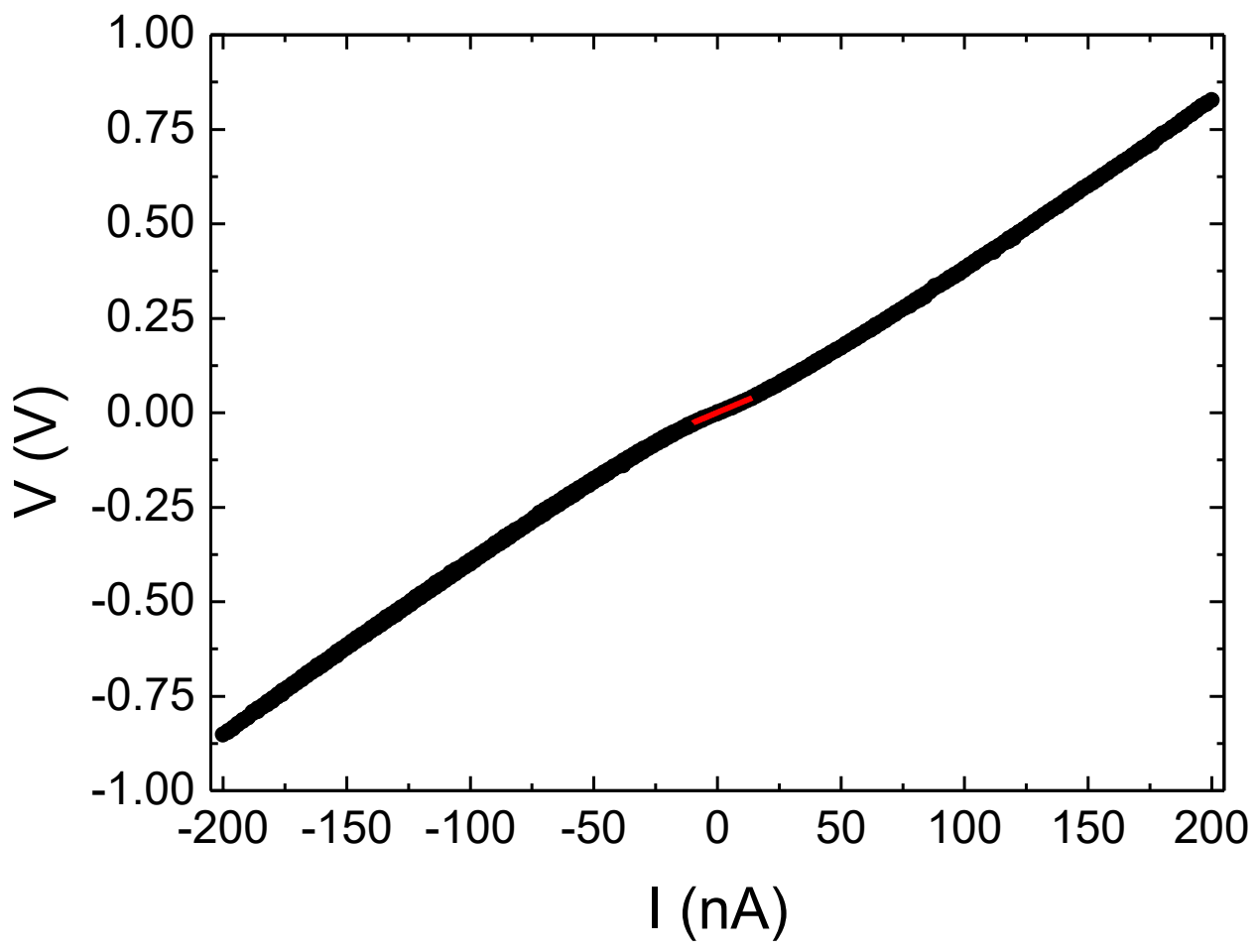

Figure S23. Device and electrical response of MUV-5a(Tb)\#2. a) Top view. b) Lateral view. c) I-V curve at 300 $\mathrm{K}$ with the corresponding linear fit (red) of the ohmic regime. The fit gives as a result a slope of $(2.75 \pm 0.02) \cdot 10^{6}$ $\Omega$ and a y-intercept value of $(0.00081 \pm 0.00018) \mathrm{V}$, with $\mathrm{R}^{2}=0.99913$. 


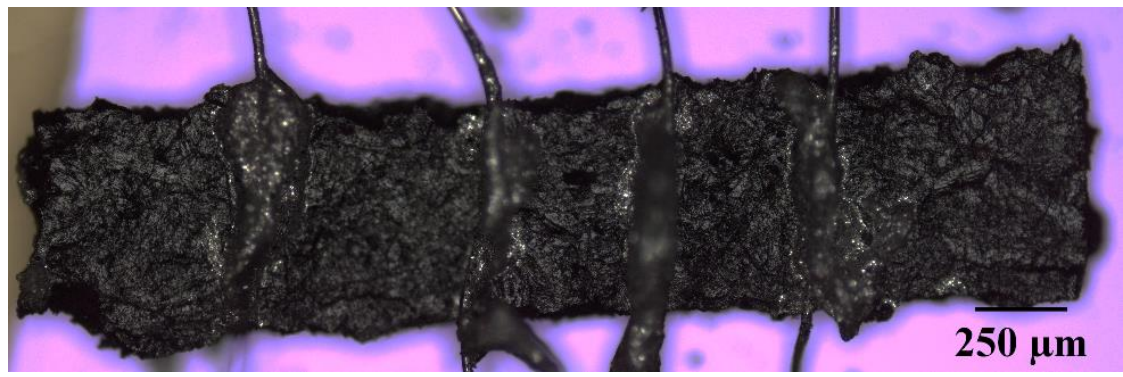

b

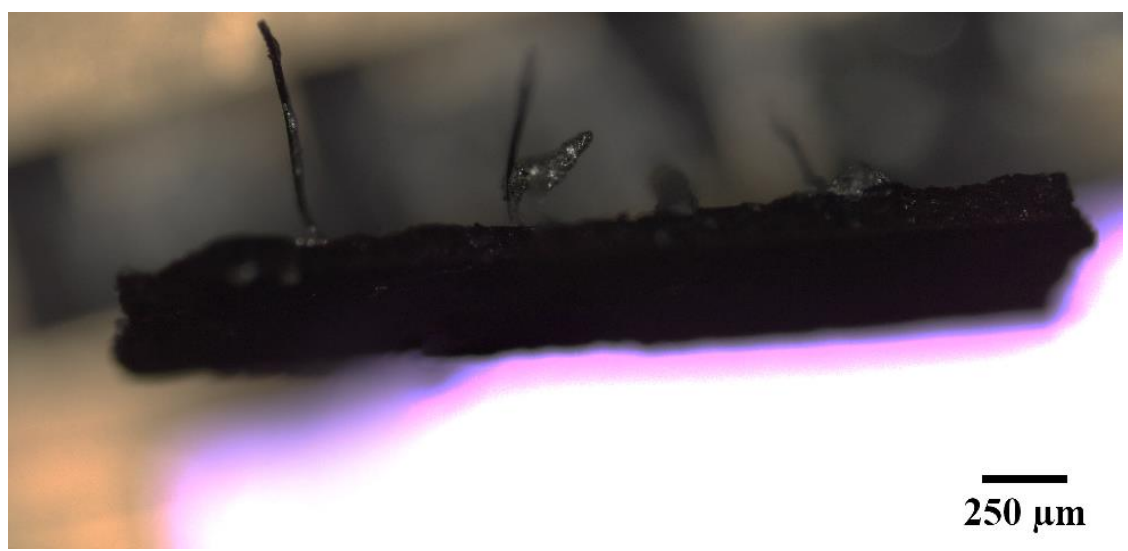

c

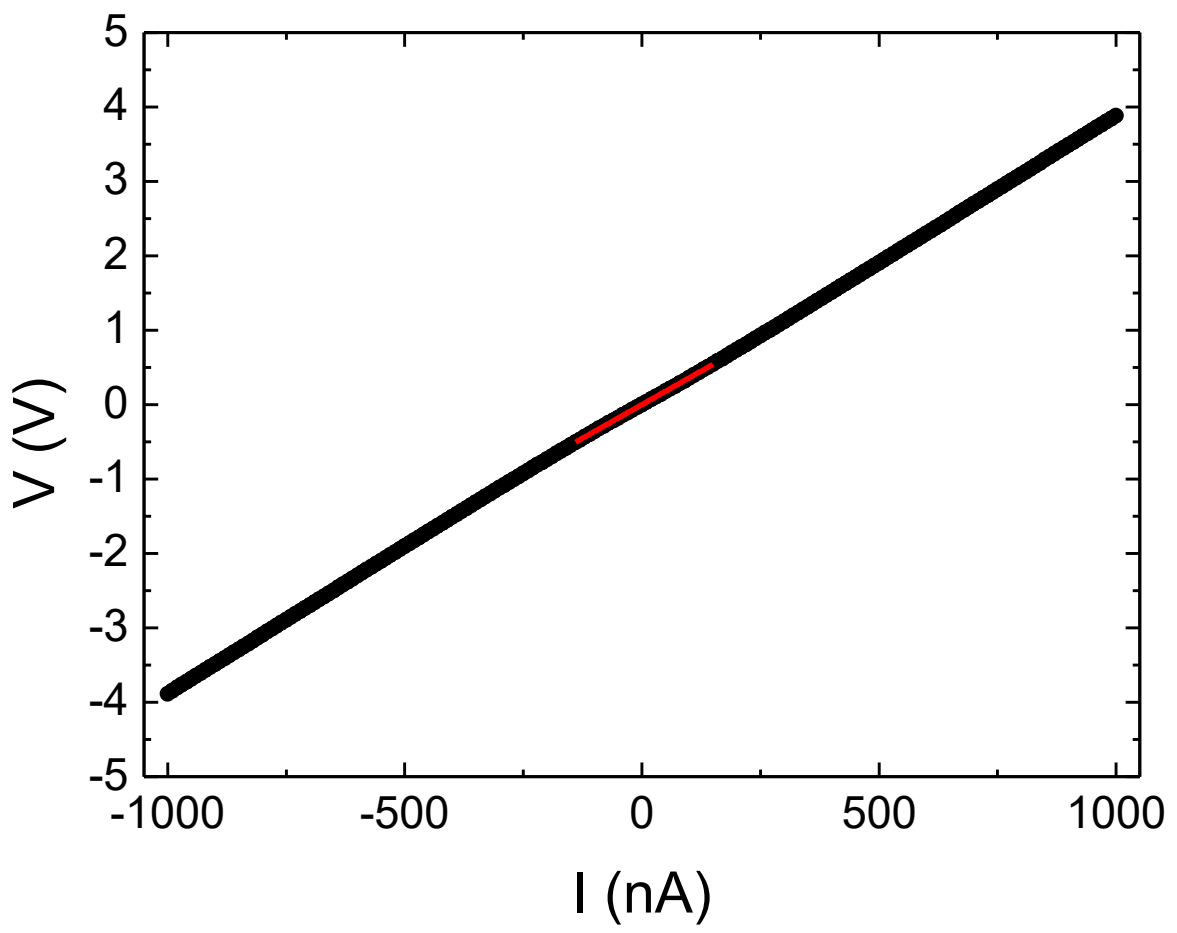

Figure S24. Device and electrical response of MUV-5a(Ho)\#1. a) Top view. b) Lateral view. c) I-V curve at 300 $\mathrm{K}$ with the corresponding linear fit (red) of the ohmic regime. The fit gives as a result a slope of $(3.604 \pm 0.005) \cdot 10^{6}$ $\Omega$ and a y-intercept value of $(-0.0004 \pm 0.0004) \mathrm{V}$, with $\mathrm{R}^{2}=0.99995$. 


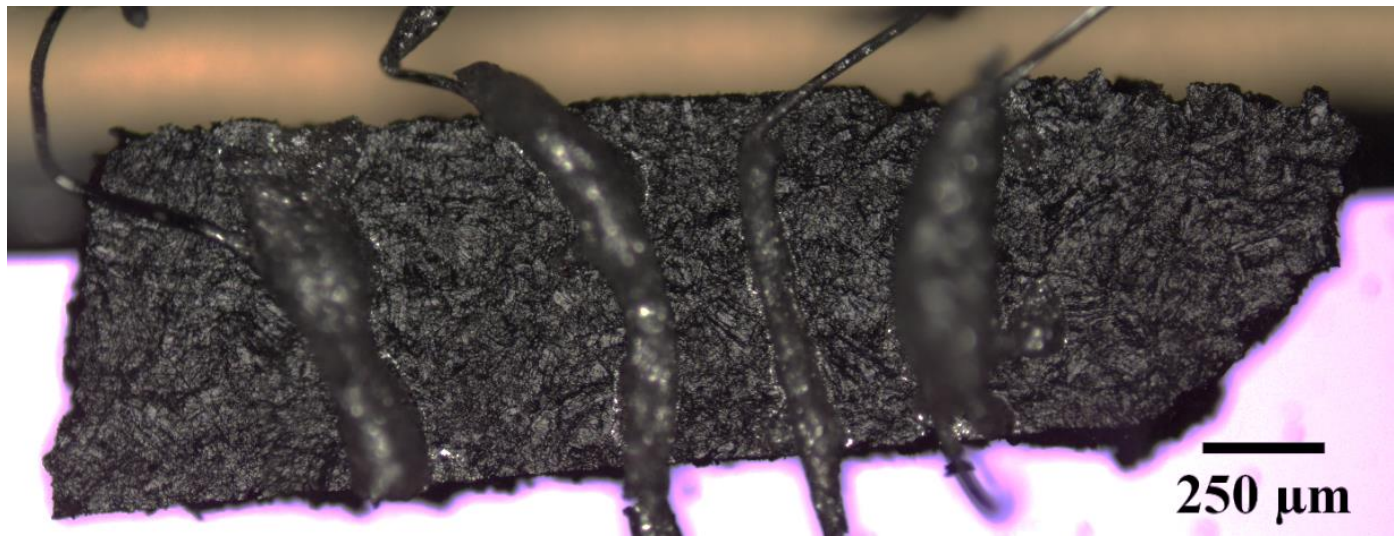

b

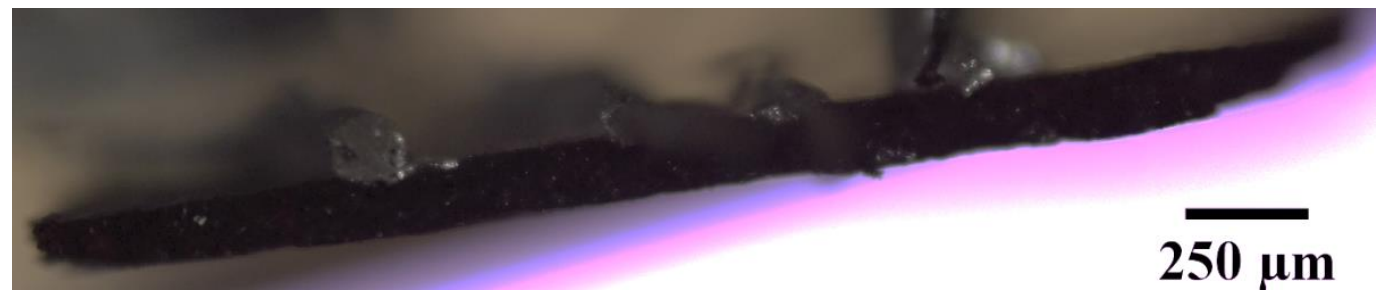

c

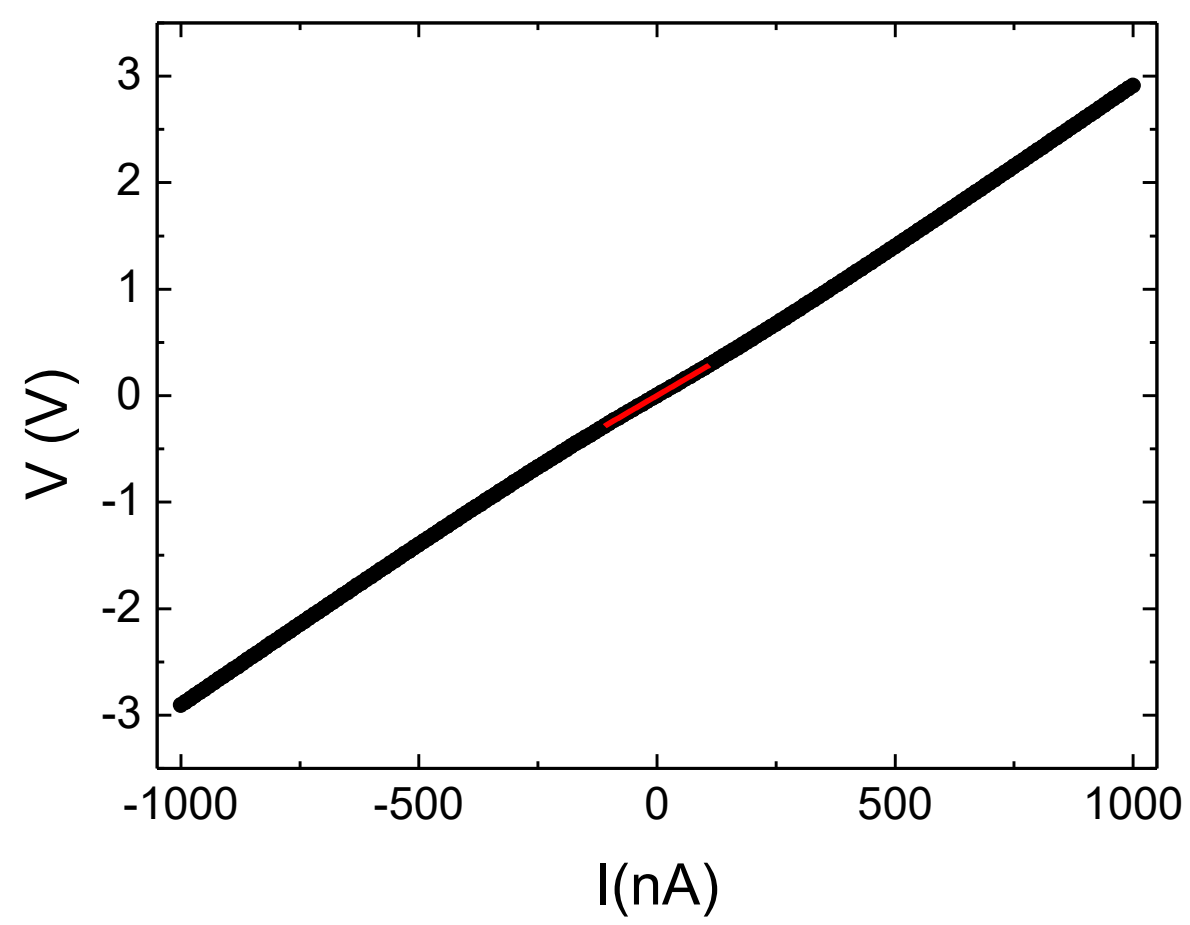

Figure S25. Device and electrical response of MUV-5a(Ho)\#2. a) Top view. b) Lateral view. c) I-V curve at 300 $\mathrm{K}$ with the corresponding linear fit (red) of the ohmic regime. The fit gives as a result a slope of $(2.602 \pm 0.002) \cdot 10^{6}$ $\Omega$ and a y-intercept value of $(0.0051 \pm 0.0015) \mathrm{V}$, with $\mathrm{R}^{2}=0.99998$. 


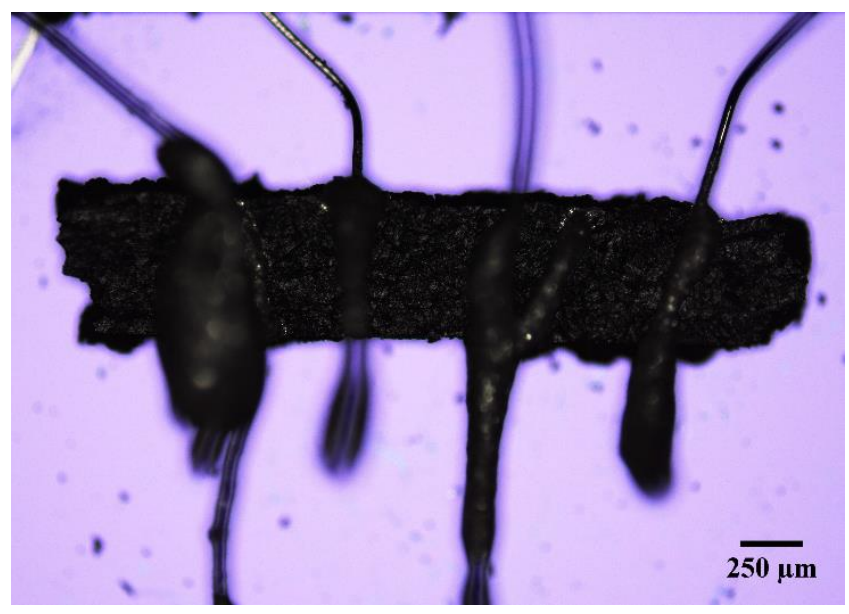

b

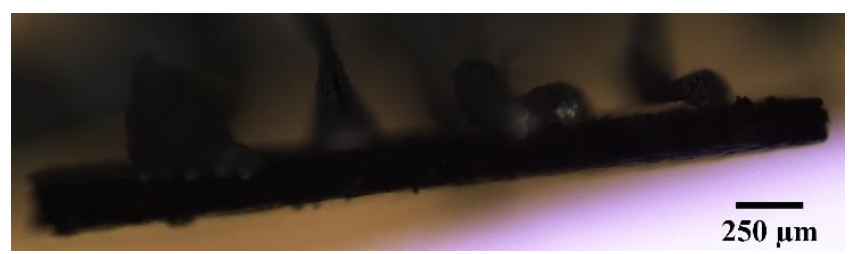

c

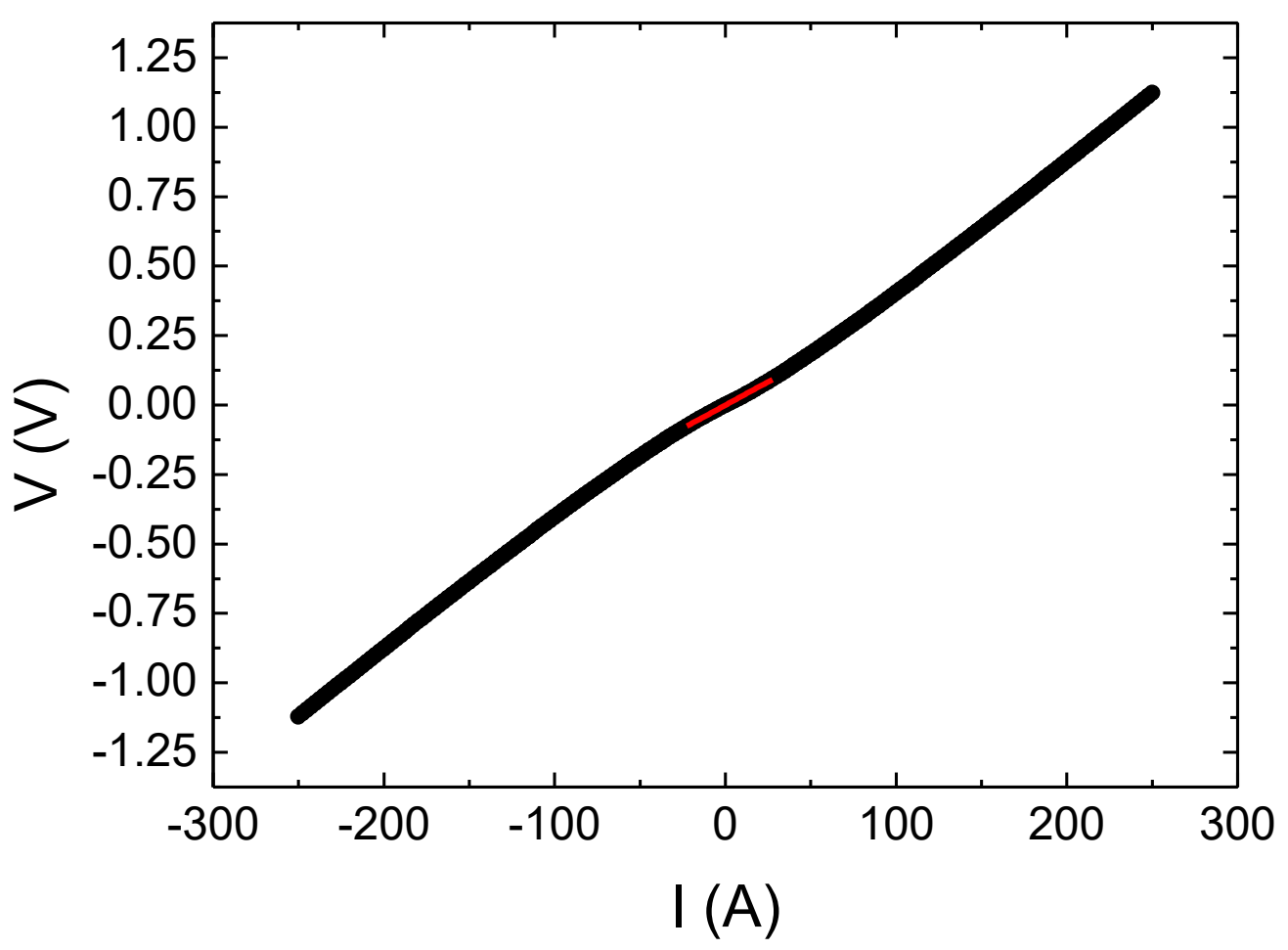

Figure S26. Device and electrical response of MUV-5a(Er)\#1. a) Top view. b) Lateral view. c) I-V curve at 300 $\mathrm{K}$ with the corresponding linear fit (red) of the ohmic regime. The fit gives as a result a slope of $(3.347 \pm 0.016) \cdot 10^{6}$ $\Omega$ and a y-intercept value of $(-0.0003 \pm 0.0002) \mathrm{V}$, with $\mathrm{R}^{2}=0.9996$. 
a

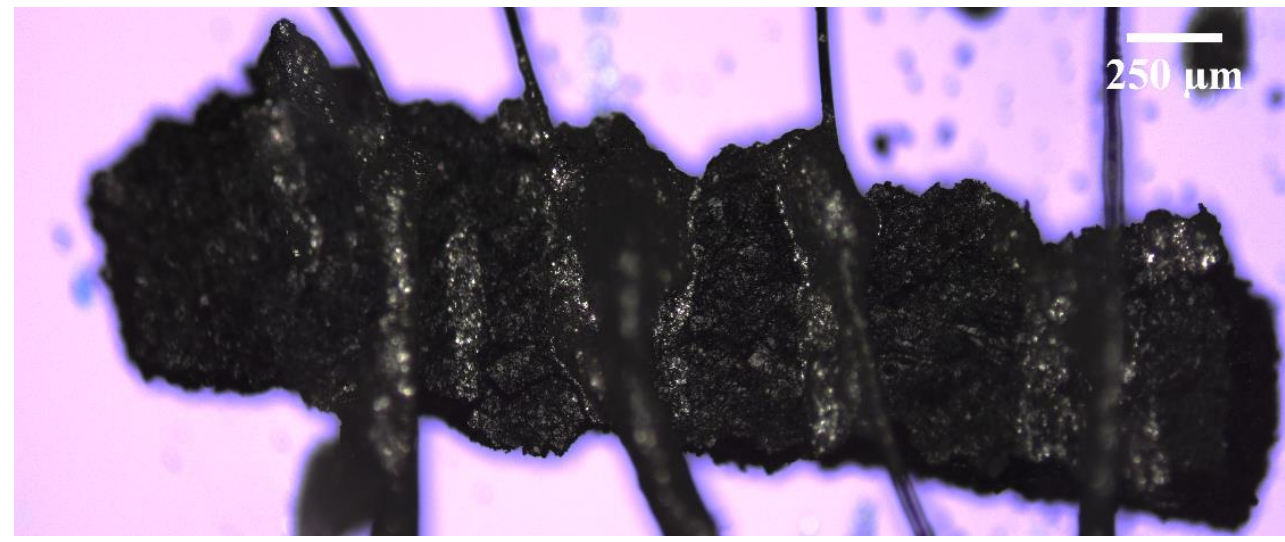

b

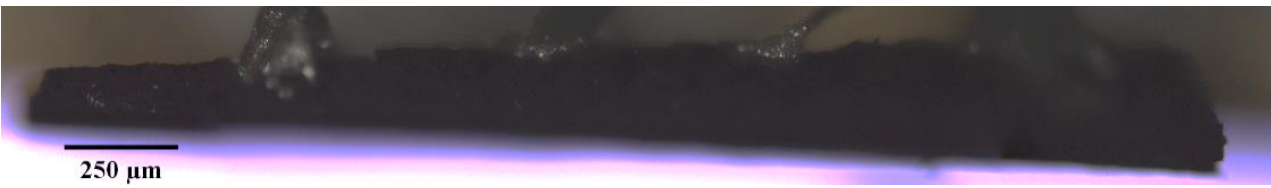

c

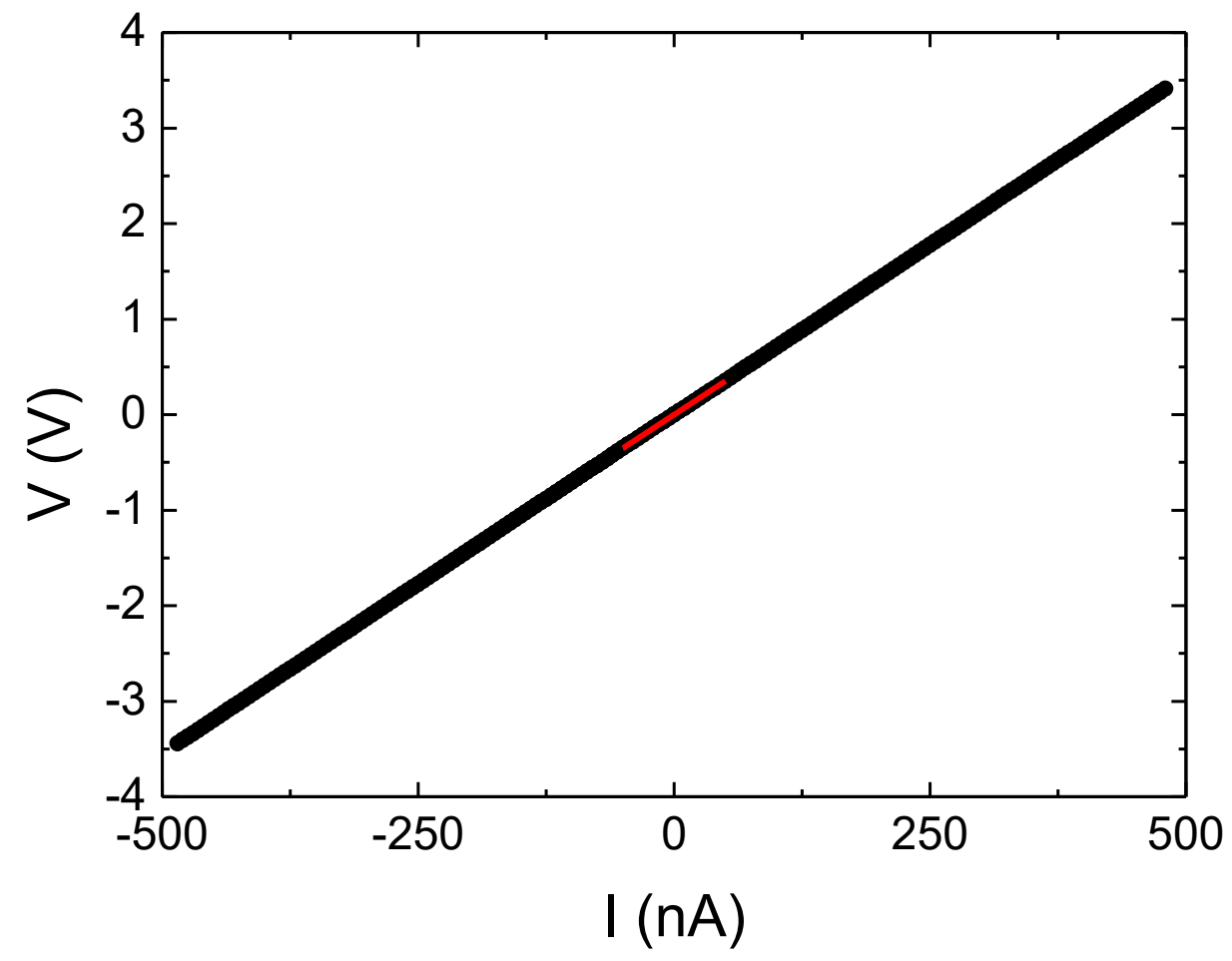

Figure S27. Device and electrical response of MUV-5a(Er)\#2. a) Top view. b) Lateral view. c) I-V curve at 300 $\mathrm{K}$ with the corresponding linear fit (red) of the ohmic regime. The fit gives as a result a slope of $(7.0164 \pm$ $0.0008) \cdot 10^{6} \Omega$ and a y-intercept value of $(-0.0017 \pm 0.0008) \mathrm{V}$, with $\mathrm{R}^{2}=0.99999$. 


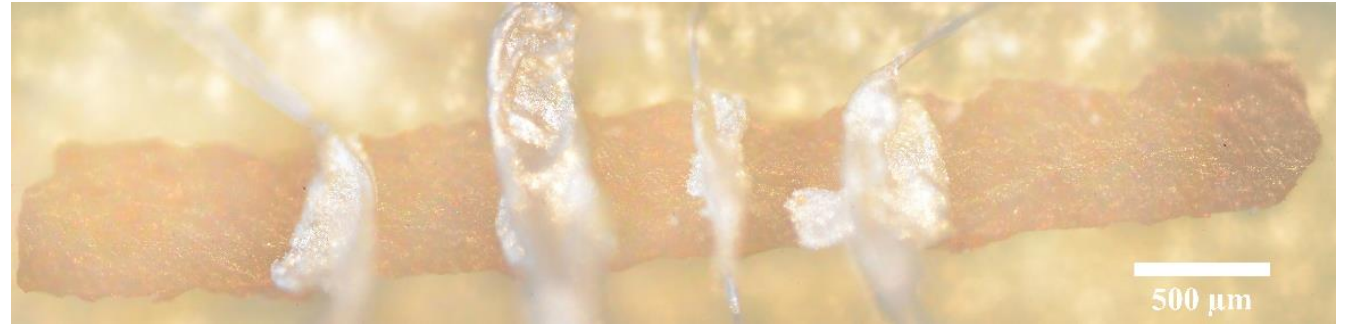

b

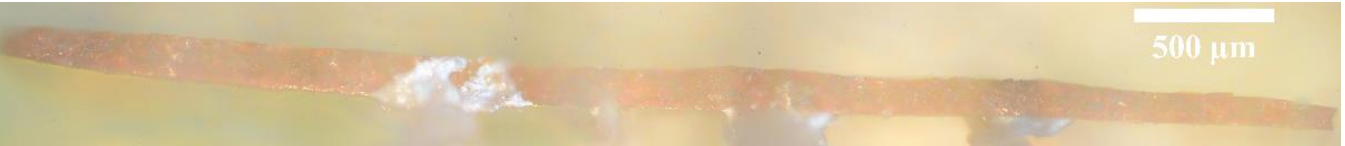

c

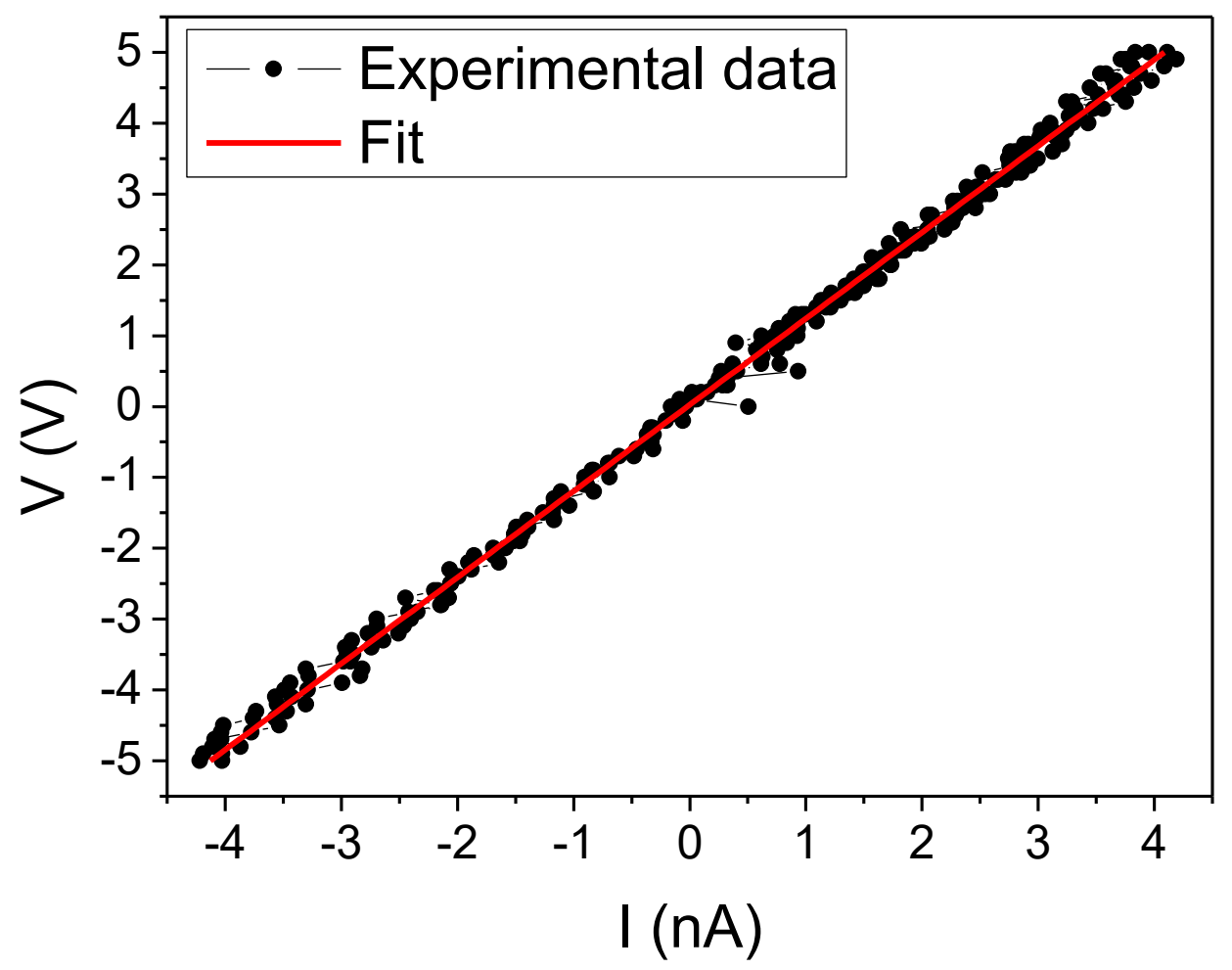

Figure S28. Device and electrical response of MUV-5b(Dy)\#1. a) Top view. b) Lateral view. c) I-V curve at 300 $\mathrm{K}$ with the corresponding linear fit (red) of the ohmic regime. The fit gives as a result a slope of $(1.214 \pm 0.004) \cdot 10^{9}$ $\Omega$ and a y-intercept value of $(0.026 \pm 0.010) \mathrm{V}$, with $\mathrm{R}^{2}=0.99688$. 
b
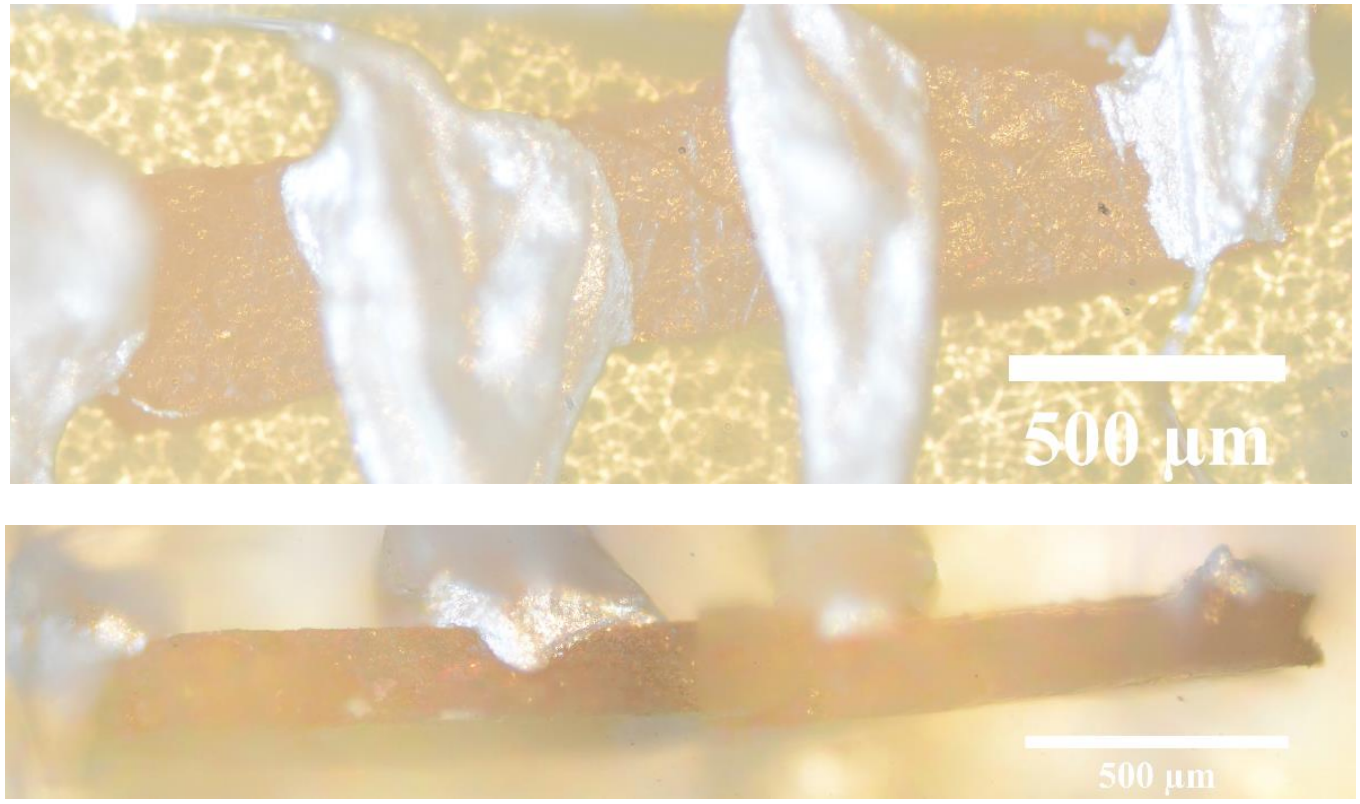

c

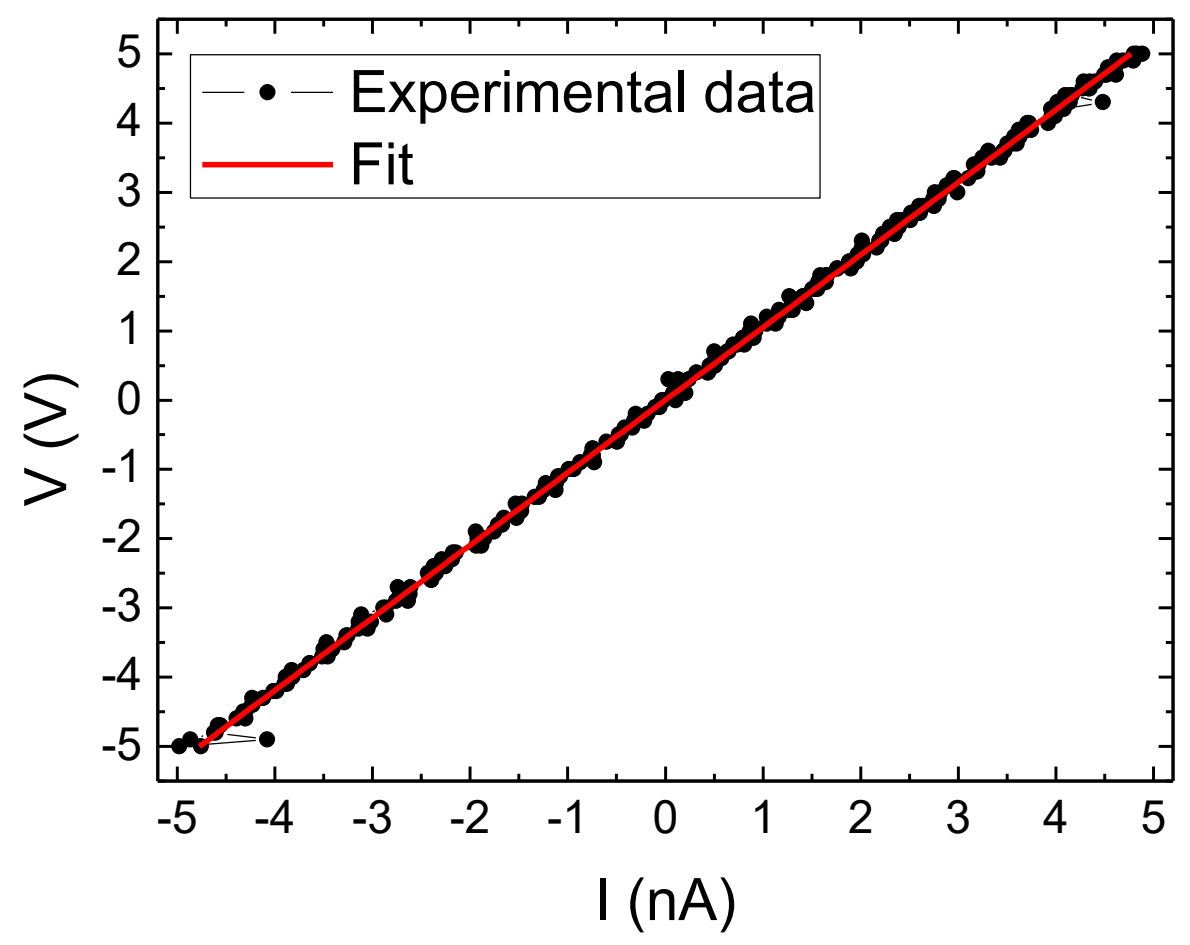

Figure S29. Device and electrical response of MUV-5b(Dy)\#2. a) Top view. b) Lateral view. c) I-V curve at 300 $\mathrm{K}$ with the corresponding linear fit (red) of the ohmic regime. The fit gives as a result a slope of $(1.0466 \pm$ $0.0019) \cdot 10^{9} \Omega$ and a y-intercept value of $(0.009 \pm 0.005) \mathrm{V}$, with $\mathrm{R}^{2}=0.99911$. 
Table S5. Charge of each moiety of TTF was estimated by using the equation $r=0.762+0.049 q$, where $r$ is the ratio between bond lengths and $\mathrm{q}$ is the charge of TTF moieties. ${ }^{5}$

\begin{tabular}{ccccc} 
Compound & Central $\mathbf{C}=\mathbf{C}(\mathbf{\AA})$ & Average $\mathbf{C}-\mathbf{S}(\mathbf{\mathbf { A }})$ & Ratio, $\mathbf{r}=\mathbf{L}(\mathbf{C}=\mathbf{C}) / \mathbf{L}(\mathbf{C}-\mathbf{S})$ & Charge, $\mathbf{q}$ \\
\hline MUV-5a(Gd) & 1.388 & 1.737 & 0.799 & $0.755( \pm 0.001)$ \\
\hline MUV-5a(Tb) & 1.342 & 1.749 & 0.767 & $0.108( \pm 0.001)$ \\
\hline MUV-5a(Dy) & 1.344 & 1.753 & 0.766 & $0.082( \pm 0.001)$ \\
\hline MUV-5a(Ho) & 1.353 & 1.749 & 0.773 & $0.224( \pm 0.001)$ \\
\hline MUV-5a(Er) & 1.341 & 1.753 & 0.765 & $0.003( \pm 0.001)$ \\
\hline MUV-5b(Dy) & 1.320 & 1.760 & 0.750 & N.A. \\
\hline
\end{tabular}

The electrochemical experiments were performed using an Autolab electrochemical workstation (Autolab-128N potentiostat/galvanostat) connected to a personal computer that uses Nova 2.1 electrochemical software. The powdered materials $\left(2 \mathrm{mg}\right.$ ) were mixed in $2 \mathrm{ml}$ of $\mathrm{CH}_{3} \mathrm{CN}$ and deposited on a $3 \mathrm{~mm}$ diameter glassy carbon disc working electrode (which was polished sequentially with $0.3,0.1$ and $0.05 \mathrm{Om}$ alumina powders and washed with deionised water before each experiment). A typical three-electrode experimental cell equipped with a platinum wire as the counter electrode, and a silver wire as the pseudoreference electrode was used for the electrochemical characterization of the working electrodes. Measurements were carried out with magnetic agitation and nitrogen bubbling. The electrochemical properties were studied measuring the $\mathrm{CV}$ at different scan rates in $0.1 \mathrm{M} \mathrm{LiClO}_{4} / \mathrm{CH}_{3} \mathrm{CN}$ solution. Ferrocene was added as an internal standard upon completion of the experiment. All potentials are reported in $\mathrm{V}$ versus $\mathrm{Fc} / \mathrm{Fc}^{+}$.

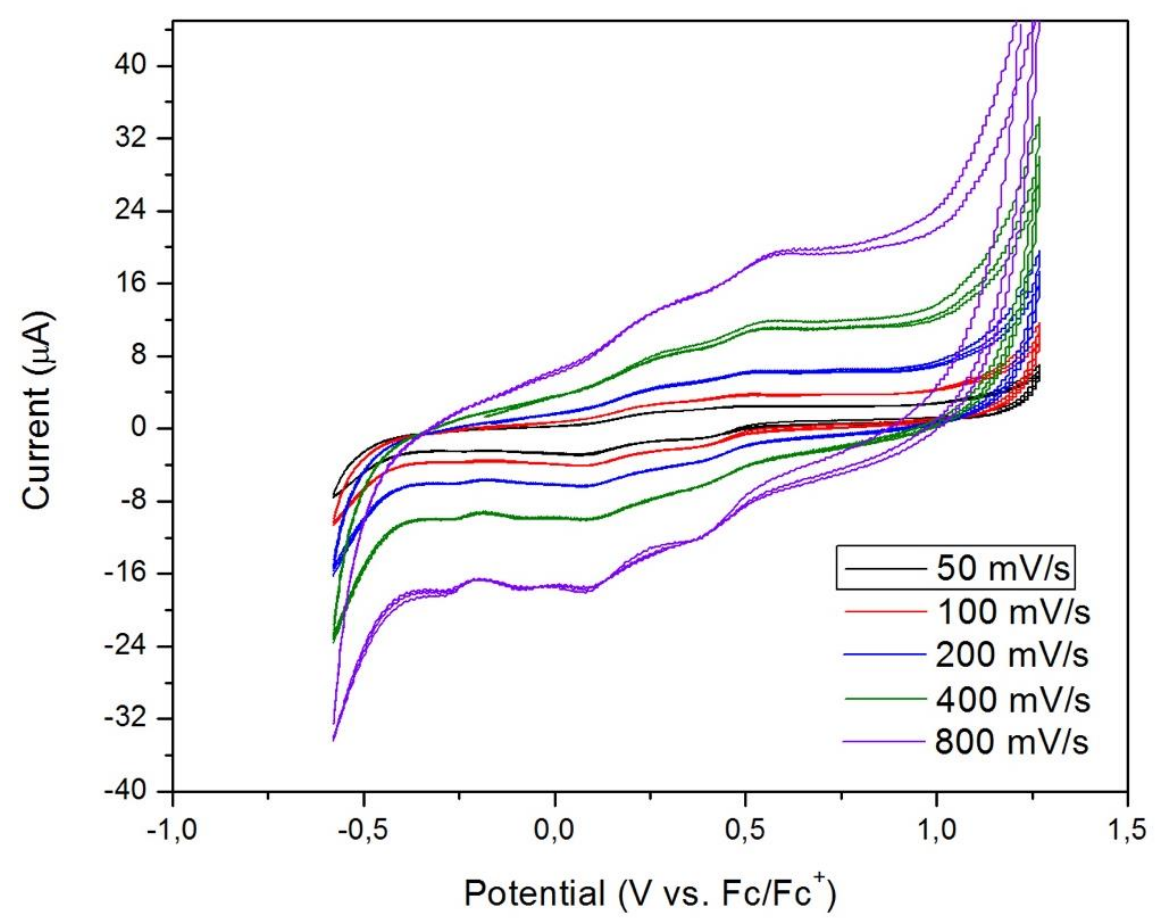

Figure S30. Solid-state cyclic voltammetry (CV) of MUV-5a(Dy) over three consecutive cycles at different scan rates $(50,100,200,400$ and $800 \mathrm{mV} / \mathrm{s})$. The experiments were performed at room temperature using Ag as reference electrode, $\mathrm{Fc}$ as internal reference and $0.1 \mathrm{M} \mathrm{LiClO} 4$ in $\mathrm{CH}_{3} \mathrm{CN}$ as electrolyte 
a)

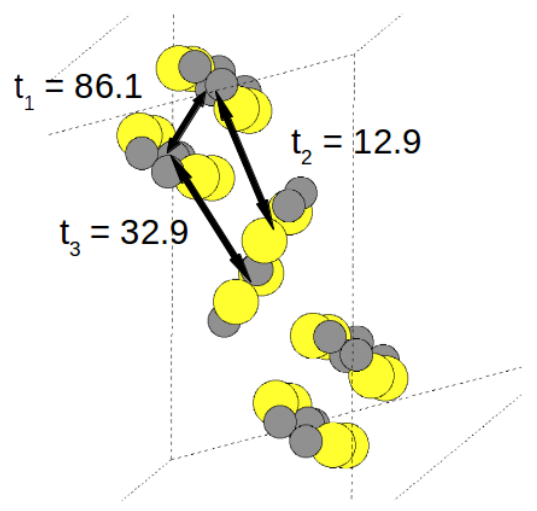

b)

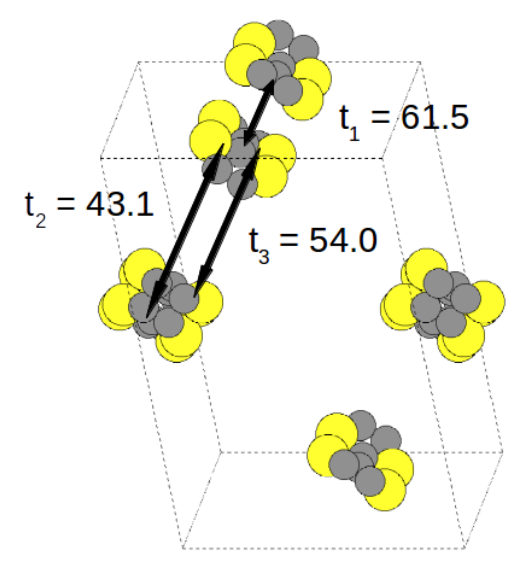

Figure S31. Schematic representation of TTF arrangements in the crystal structures of a) ref. [6] and b) ref. [7] indicating the transfer integrals.

Table S6. Calculated transfer integrals using B3LYP and PBE0 hybrid functionals

\begin{tabular}{|c|c|c|}
\hline & \multicolumn{2}{|c|}{ Transfer integrals (meV) } \\
\hline Structure & B3LYP & PBE0 \\
\hline MUV-5a & 16.5 & 15.5 \\
\hline & 90.6 & 93.3 \\
\hline MUV-5b & 8.4 & 9 \\
\hline & 60.5 & 57.8 \\
\hline Ref. [6] & 86.1 & 85.4 \\
\hline & 32.9 & 27.1 \\
\hline & 12.9 & 10.2 \\
\hline Ref. [7] & 61.5 & 85.2 \\
\hline & 54 & 40 \\
\hline & 43.1 & 29.1 \\
\hline
\end{tabular}




\section{S7. Photoluminescence}

\section{Synthesis and characterisation of MUV-5a(Y $\left.\mathbf{b}_{0.76} \mathrm{Er}_{0.24}\right)$}

Synthesis of MUV-5a(Ybo.76Ero.24): $12 \mu \mathrm{mol}$ of $\mathrm{Yb}\left(\mathrm{CH}_{3} \mathrm{COO}\right)_{3}(4.6 \mathrm{mg}), 4 \mu \mathrm{mol} \mathrm{Er}\left(\mathrm{CH}_{3} \mathrm{COO}\right)_{3}(1.4 \mathrm{mg})$ and $12.5 \mu \mathrm{mol}$ of $\mathrm{H}_{4}$ TTFTB were suspended in a mixture of $1.3 \mathrm{~mL}$ of $\mathrm{H}_{2} \mathrm{O}$ and $0.7 \mathrm{~mL}$ of acetic acid (AcOH) in a 4 $\mathrm{mL}$ glass vial. The vial was sealed and sonicated for a few minutes to get a homogeneous suspension. The dark suspension was subsequently heated in an oven at $170{ }^{\circ} \mathrm{C}$ for 12 hours $\left(\uparrow+2.0^{\circ} \mathrm{C} \min ^{-1}, \downarrow-0.4{ }^{\circ} \mathrm{C} \mathrm{min}{ }^{-1}\right)$. After cooling down to room temperature, the dark red crystals were recovered by centrifugation and rinsed with fresh $\mathrm{DMF}$, water and $\mathrm{MeOH}$ several times. The solids were then allowed to dry in air at room temperature. The solids were further heated at $150{ }^{\circ} \mathrm{C}$ for at least 2 hours.
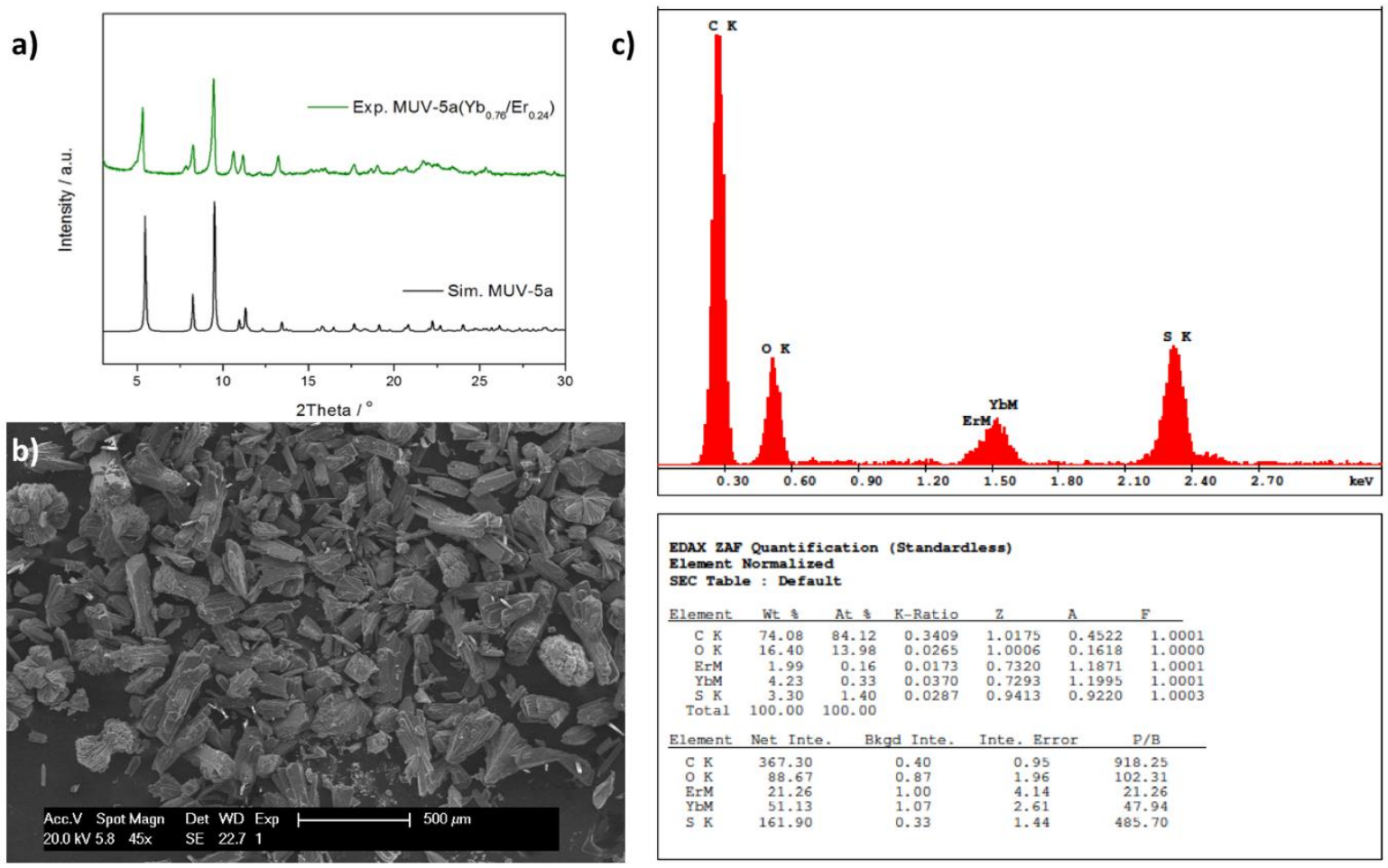

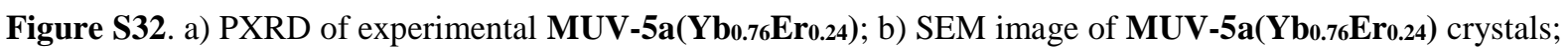
and c) EDS analysis of MUV-5a(Y $\left.\mathbf{b}_{0.76} \mathbf{E r}_{0.24}\right)$.

Table S7. Determination of Yb/Er ratio in MUV-5a(Yb/Er) by ICP-MS (Agilent 7900). The molecular formula was calculated to be MUV-5a(Ybo.76Ero.24).

\begin{tabular}{|c|c|}
\hline Description & MUV-5(Yb/Er) \\
\hline $\mathrm{Er}(\mathrm{mg} / \mathrm{g})$ & $42.7 \pm 0.8$ \\
\hline $\mathrm{Yb}(\mathrm{mg} / \mathrm{g})$ & $140 \pm 2$ \\
\hline
\end{tabular}


Emission and excitation spectra were recorded on a modular double grating excitation spectrofluorimeter with a TRIAX 320 emission monochromator (Fluorolog-3, Horiba Scientific) coupled to a near infrared H9170 Hamamatsu photomultipliers, using the front face acquisition mode. The excitation source was a $450 \mathrm{~W}$ Xe arc lamp. The excitation spectra were corrected for the spectral distribution of the lamp intensity using a photodiode reference detector. Time-resolved measurements were carried out with pulsed Xe-Hg lamp excitation, in front face acquisition mode. The low temperature measurements $(12 \mathrm{~K})$ were performed using a helium-closed cycle cryostat with vacuum system measuring ca. $5 \times 10^{-6}$ mbar and a Lakeshore 330 auto-tuning temperature controller with a resistance heater.

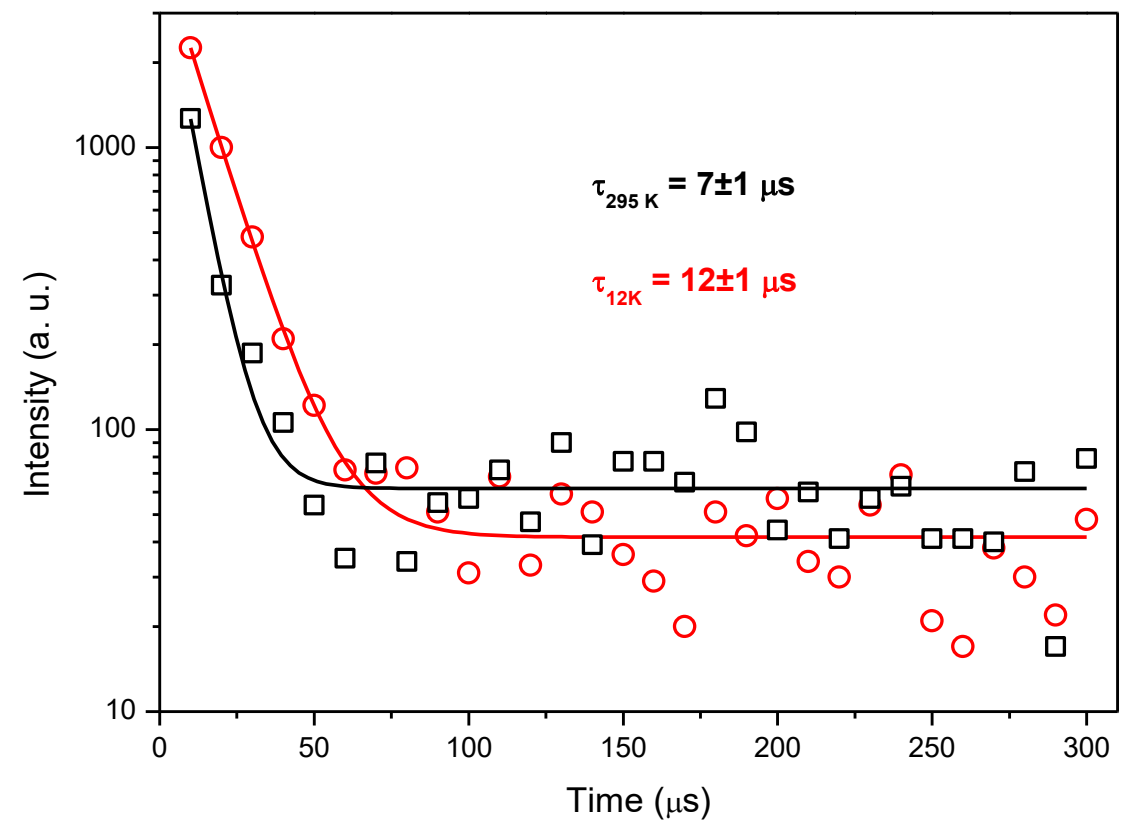

Figure S33. ${ }^{2} \mathrm{~F}_{7 / 2}$ decay curve of MUV-5a( $\left.\mathbf{Y} \mathbf{b}_{\mathbf{0 . 7 6}} \mathbf{E r}_{\mathbf{0 . 2 4}}\right)$ acquired at $295 \mathrm{~K}$ (black) and at $12 \mathrm{~K}$ (red) monitoring the $\mathrm{Yb}^{3+}$ emission at $980 \mathrm{~nm}$ with the excitation fixed at $560 \mathrm{~nm}$. The solid lines are the best fit using a single exponential decay function $\left(r^{2}>0.98\right.$ and 0.99 for $295 \mathrm{~K}$ and $12 \mathrm{~K}$, respectively). Note that the minimum time discrimination of the phosphorimeter used is $10 \mu \mathrm{s}$. 


\section{S8. Magnetic Measurements}

Magnetic measurements were performed with a Quantum Design Physical Property Measurement System (PPMS). Variable-temperature $(2-300 \mathrm{~K})$ direct current $(\mathrm{dc})$ magnetic susceptibility measurements were carried out in an applied field of $1.0 \mathrm{kOe}$ and variable field magnetization measurements up to $5 \mathrm{~T}$ at $2.0 \mathrm{~K}$. Variable-temperature $(2-15 \mathrm{~K})$ alternating current (ac) magnetic susceptibility measurements in a $\pm 4.0 \mathrm{G}$ oscillating field at frequencies in the range of $1-997 \mathrm{~Hz}$ were carried out in a zero dc field and in a dc field of $1.0 \mathrm{kOe}$.

\section{AC Measurements}
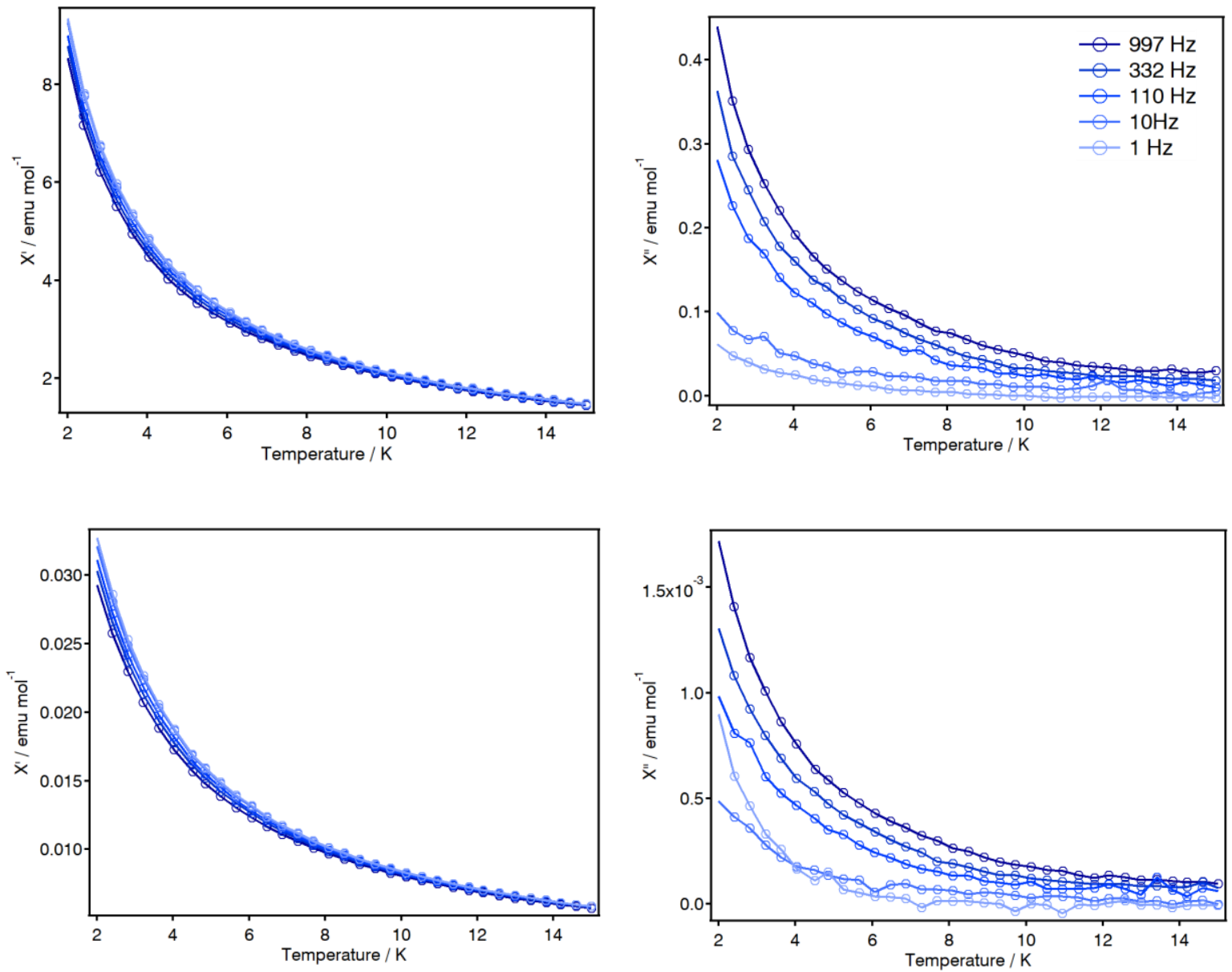

Figure S34. In-phase (left) and out-of-phase (right) dynamic magnetic susceptibility of MUV-5a(Dy) under an external magnetic field of $0 \mathrm{G}$ (top) and $1000 \mathrm{G}$ (bottom). 

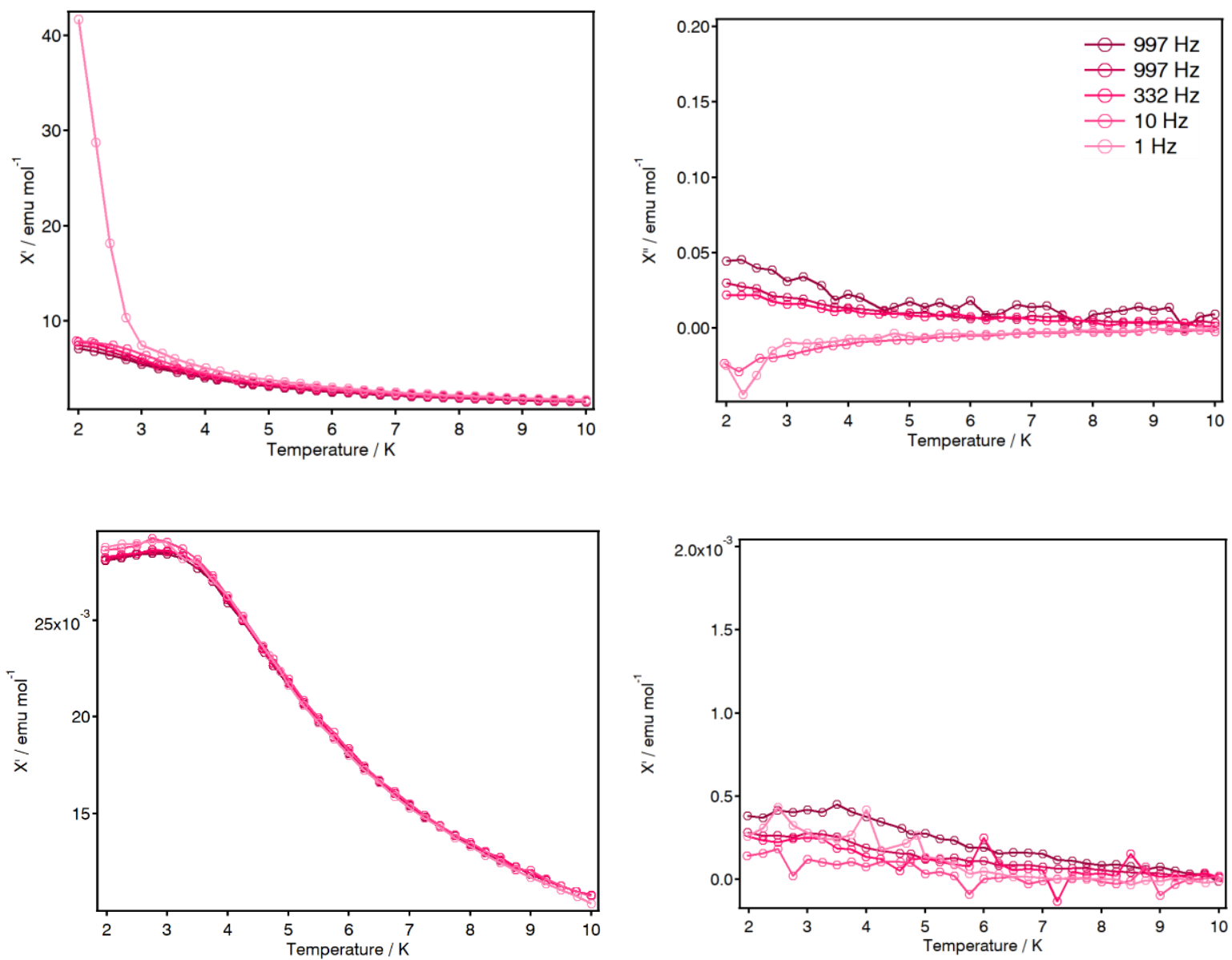

Figure S35. In-phase (left) and out-of-phase (right) dynamic magnetic susceptibility of MUV-5a(Er) under an external magnetic field of $0 \mathrm{G}$ (top) and $1000 \mathrm{G}$ (bottom). 


\section{Computational Modelling}

Radial Effective Charge (REC) model: The model is an electrostatic semi-empirical crystal field approach commonly used in molecular magnetism, which provides an estimation of the crystal field parameters (CFPs) and permits to rationalize the magnetic properties of a particular $f$-block coordination complex. ${ }^{8}$ From the computed CFPs, the model estimates the ground- $J$ multiplet energy levels and their corresponding wave functions, which are composed by the different $M_{J}$ microstates. This kind of calculations use the crystallographic atomic coordinates of the first coordination sphere around the magnetic centre as an input. The software code that has this model implemented is the SIMPRE computational package, ${ }^{9}$ which parameterizes the electric field effect produced by the surrounding ligands by using the following Crystal Field Hamiltonian expressed in terms of the Extended Stevens Operators (ESOs). ${ }^{10}$

$$
\hat{H}_{c f}(J)={ }_{k=2,4,6 q=k}^{k} B_{k}^{q} O_{k}^{q}={ }_{k=2,4,6 q=k}^{k} a_{k}\left(1 \quad{ }_{k}\right) A_{k}^{q}\left\langle r^{k}\right\rangle O_{k}^{q}
$$

where $k$ is the order (also called rank or degree) and $q$ is the operator range, that varies between $k$ and $-k$, of the Stevens operator equivalents $O_{k}^{q}$ as defined by Ryabov in terms of the angular momentum operators $J_{ \pm}$and $J_{z}$, ${ }^{11}$ where the components $O_{k}^{q}(c)$ and $O_{k}^{q}(s)$ correspond to the ESOs with q $\geq 0$ and q $<0$ respectively. ${ }^{12}$ Note that all the Stevens CF parameters $B_{k}^{q}$ are real, whereas the matrix elements of $O_{k}^{q}(\mathrm{q}<\mathrm{O})$ are imaginary. $a_{k}$ are the $\alpha, \beta$ and $\gamma$ Stevens coefficientsfor $k=2,4,6$, respectively, which are tabulated and depend on the number of $f$ electrons. $\sigma_{\mathrm{k}}$ are the Sternheimer shielding parameters of the $4 f$ electronic shell, and $\left\langle r^{k}\right\rangle$ are the expectation values of the radius. $^{13}$

In SIMPRE, the $A_{k}^{q} \mathrm{CF}$ parameters are determined by the following relations:

$$
\begin{array}{ll}
A_{k}^{0}=\frac{4 \pi}{2 k+1} \sum_{i=1}^{N} \frac{Z_{i} e^{2}}{R_{i}^{k+1}} Z_{k 0}\left(\theta_{i}, \varphi_{i}\right) p_{k q} & \\
A_{k}^{q}=\frac{4 \pi}{2 k+1} \sum_{i=1}^{N} \frac{Z_{i} e^{2}}{R_{i}^{k+1}} Z_{k q}^{c}\left(\theta_{i}, \varphi_{i}\right) p_{k q} & (\mathrm{q}>0) \\
A_{k}^{q}=\frac{4 \pi}{2 k+1} \sum_{i=1}^{N} \frac{Z_{i} e^{2}}{R_{i}^{k+1}} Z_{k|q|}^{s}\left(\theta_{i}, \varphi_{i}\right) p_{k|q|} & (\mathrm{q}<0)
\end{array}
$$

where $R_{i}, \theta_{i}$ and $\varphi_{i}$ are the effective polar coordinates of the point charges, and $Z_{i}$ is the effective point charge, associated to the $i$-th donor atom with the lanthanoid at the origin, $N$ is the number of ligands; $e$ is the electron charge, $p_{k q}$ are the prefactors of the spherical harmonics and $\mathrm{Z}_{\mathrm{kq}}$ are the tesseral harmonics expressed in terms of the polar coordinates for the i-th donor atom.

In the model, the effect of the ligand is reproduced through an effective point charge situated between the lanthanoid and the coordinated atom at a distance $R_{i}$ from the magnetic centre, which is smaller than the real metal-ligand distance $\left(\mathrm{r}_{\mathrm{i}}\right)$. To account for the effect of covalent electron sharing, a radial displacement vector $\left(D_{r}\right)$ is defined, in which the polar coordinate $r$ of each coordinated atom is collectively varied, $R_{i}=r_{i}-D_{r}$, whereas $\theta_{i}$ and $\varphi_{i}$ remain constant. In the case of MUV-5a, we have taken advantage of the REC parameters recently obtained for the coordinated $\mathrm{O}$ atoms of the carboxylate ligands in MUV-4b $\left(D_{r}=0.885 \AA, Z_{i}=0.1024\right),{ }^{14}$ which have allowed a satisfactory reproduction of the magnetic properties of the series by considering the different crystallographic centres. The relative error $E$ is defined as:

$$
E=\frac{1}{n} \sum_{i=1}^{n} \frac{\left[\chi_{\text {theo }, i}-\chi_{\exp , i}\right]^{2}}{\left[\chi_{\text {exp }, i}\right]^{2}}
$$


where $\chi_{\text {exp }}$ and $\chi_{\text {theo }}$ are experimental and theoretical magnetic susceptibility, respectively, and $n$ is the number of points.

Table S8. Crystal-field parameters $\left(A_{k}^{q}\left\langle r^{k}\right\rangle\right.$; Stevens notation) in $\mathrm{cm}^{-1}$ obtained for the two crystallographically independent magnetic centers of MUV-5a(Ln), Ln = Tb, Dy, Ho and Er.

\begin{tabular}{|c|c|c|c|c|c|c|c|c|c|}
\hline $\boldsymbol{k}$ & $q$ & Tb1 & Tb2 & Dy1 & Dy2 & Ho1 & Ho2 & Er1 & Er2 \\
\hline 2 & 0 & -123.38 & -110.40 & -97.52 & 58.41 & 78.89 & 22.71 & -99.46 & -74.71 \\
\hline 2 & 1 & 85.44 & 128.36 & 40.72 & -149.32 & -4.62 & -237.28 & -10.25 & -159.29 \\
\hline 2 & -1 & -93.15 & -42.03 & -162.48 & -314.11 & 6.81 & -272.58 & 110.45 & 152.46 \\
\hline 2 & 2 & 1.57 & -111.35 & 23.29 & -0.41 & 111.82 & 41.45 & 47.09 & -69.12 \\
\hline 2 & -2 & 17.88 & -15.68 & 12.41 & -130.83 & 94.85 & -59.23 & 8.96 & 26.57 \\
\hline 4 & 0 & -16.09 & -69.61 & 94.78 & 56.87 & 37.09 & 59.29 & 69.63 & -55.83 \\
\hline 4 & 1 & -28.26 & -166.73 & 19.64 & 28.23 & -0.90 & 44.39 & -26.79 & 76.40 \\
\hline 4 & -1 & 30.80 & 56.15 & -75.69 & 140.78 & 14.71 & -45.70 & 277.66 & 139.40 \\
\hline 4 & 2 & -45.03 & 88.69 & 40.51 & 47.03 & 278.15 & -23.39 & -77.04 & 258.56 \\
\hline 4 & -2 & -509.52 & -197.16 & 22.01 & 8.21 & 124.62 & -70.58 & -14.70 & 240.92 \\
\hline 4 & 3 & 414.81 & 1323.63 & 814.65 & -97.90 & -57.76 & 27.05 & -282.42 & -484.32 \\
\hline 4 & -3 & 317.20 & 541.15 & -891.09 & -45.55 & -14.75 & 426.03 & 972.37 & 395.01 \\
\hline 4 & 4 & 178.80 & -55.24 & 40.28 & 429.56 & -466.75 & -367.98 & -9.16 & -73.04 \\
\hline 4 & -4 & -31.79 & -26.588 & 60.79 & -405.88 & -49.62 & 442.27 & -3.85 & 154.45 \\
\hline 6 & 0 & -23.97 & 8.73 & 16.60 & -13.01 & 0.77 & -15.48 & 9.93 & 1.51 \\
\hline 6 & 1 & 5.76 & 11.42 & -1.84 & -56.44 & 6.86 & 23.26 & -7.22 & 15.11 \\
\hline 6 & -1 & -6.26 & -80.98 & 8.31 & -40.24 & -0.42 & 1.24 & 75.30 & -29.21 \\
\hline 6 & 2 & 4.11 & -101.86 & -14.46 & -22.06 & 106.23 & 2.91 & 37.25 & -20.13 \\
\hline 6 & -2 & 46.61 & 70.67 & -7.95 & 5.37 & -55.50 & 34.36 & 6.91 & -8.69 \\
\hline 6 & 3 & 39.97 & 185.76 & -230.00 & -51.07 & 2.36 & -45.69 & 79.18 & -262.90 \\
\hline 6 & -3 & 30.53 & 34.23 & 251.74 & -24.97 & 15.18 & -8.13 & -272.90 & 146.59 \\
\hline 6 & 4 & -104.64 & -39.59 & -51.94 & -71.74 & 146.22 & 63.97 & -29.09 & -24.07 \\
\hline 6 & -4 & 18.61 & 101.89 & -78.39 & 40.71 & 32.42 & -103.90 & -11.36 & 52.53 \\
\hline 6 & 5 & 204.14 & 161.93 & 185.29 & -633.87 & -15.54 & 474.37 & -47.93 & -189.91 \\
\hline 6 & -5 & -321.95 & 60.38 & -65.27 & -102.96 & 9.14 & 82.19 & 94.30 & 56.23 \\
\hline 6 & 6 & 24.58 & 12.55 & -3.56 & -36.04 & 111.95 & 0.56 & -7.36 & -12.66 \\
\hline 6 & -6 & 90.91 & -27.40 & -39.95 & -25.03 & 6.41 & -29.54 & -4.66 & 51.30 \\
\hline
\end{tabular}

Table S9. Ground multiplet energy level scheme (in $\mathrm{cm}^{-1}$ ) and main $\left|\mathrm{M}_{\mathbf{J}}\right\rangle$ contributions (> 10\%) to the wave function calculated for MUV-5a(Tb1) and MUV-5a(Tb2).

\begin{tabular}{|c|c|c|c|}
\hline \multicolumn{2}{|r|}{ MUV-5a(Tb1) } & \multicolumn{2}{|r|}{ MUV-5a(Tb2) } \\
\hline $\mathbf{0}$ & $59.3 \%|0>+19.0 \%|+2>+19.0 \%|-2\rangle$ & $\mathbf{0 . 0}$ & $66.9 \% \mid 0>$ \\
\hline 0.3 & $46.1 \%|+1>+46.1 \%|-1>$ & 2.6 & $41.9 \%|+1>+41.9 \%|-1\rangle$ \\
\hline 60 & $18.5 \%|+4>+18.5 \%|-4>$ & 48 & $30.5 \%|+1>+30.5 \%|-1>$ \\
\hline 63 & $\begin{array}{l}21.9 \%|+2>+21.9 \%|-2>+17.4 \% \mid+4> \\
+17.4 \% \mid-4>\end{array}$ & 66 & $36.2 \%|+2>+36.2 \%|-2>$ \\
\hline 69 & $\begin{array}{l}19.6 \%|+3>+19.6 \%|-3\rangle+14.0 \% \mid+5> \\
+14.0 \% \mid-5>\end{array}$ & 86 & $26.2 \%|+2>+26.2 \%|-2>$ \\
\hline 72 & $\begin{array}{l}29.7 \%|+3>+29.7 \%|-3>+16.3 \% \mid+5> \\
+16.3 \% \mid-5>\end{array}$ & 102 & $\begin{array}{l}20.1 \%|+3>+20.1 \%|-3>+19.9 \% \mid+6>+ \\
19.9 \% \mid-6>\end{array}$ \\
\hline 113 & $34.4 \%|+1>+34.4 \%|-1>$ & 113 & $29.5 \%|+6>+29.5 \%|-6>$ \\
\hline 123 & $\begin{array}{l}26.3 \%|+2>+26.3 \%|-2>+13.5 \% \mid+4> \\
+13.5 \% \mid-4>\end{array}$ & 153 & $21.8 \%|+3>+21.8 \%|-3>$ \\
\hline 134 & $23.9 \%|0>+17.3 \%|+2>+17.3 \% \mid-2>$ & 164 & $\begin{array}{l}20.5 \%|+3>+20.5 \%|-3>+16.2 \% \mid+6>+ \\
16.2 \%|-6>+10.4 \%|+4>+10.4 \% \mid-4>\end{array}$ \\
\hline
\end{tabular}


 $26.2 \%|+5>+26.2 \%|-5>+13.5 \% \mid+3>$ $+13.5 \% \mid-3>$
$19125.0 \%|+5>+25.0 \%|-5>+12.7 \% \mid+3>$ $+12.7 \% \mid-3>$
$25626.0 \%|+6>+26.0 \%|-6>+13.8 \% \mid+4>$ $+13.8 \% \mid-4>$
$25726.0 \%|+6>+26.0 \%|-6>+14.3 \% \mid+4>$ $+14.3 \% \mid-4>$

$20419.8 \%|+4>+19.8 \%|-4>+12.0 \% \mid+5>+$ $12.0 \% \mid-5>$

$20822.6 \%|+4>+22.6 \%|-4>+11.7 \% \mid+5>+$ $11.7 \%|-5\rangle$

$25324.8 \%|+5>+24.8 \%|-5>+11.2 \% \mid+4>+$ $11.2 \% \mid-4>$

$25324.7 \%|+5>+24.7 \%|-5>+11.5 \% \mid+4>+$ $11.5 \% \mid-4>$

Table S10. Ground multiplet energy level scheme (Kramers doublets in $\mathrm{cm}^{-1}$ ) and main $\left|\mathrm{M}_{\mathrm{J}}\right\rangle$ contributions (> $10 \%)$ to the wave function calculated for MUV-5a(Dy1) and MUV-5a(Dy2).

\begin{tabular}{|c|c|c|c|}
\hline \multicolumn{2}{|r|}{ MUV-5a(Dy1) } & \multicolumn{2}{|r|}{ MUV-5a(Dy2) } \\
\hline $\mathbf{0}$ & $98.7 \% \mid \pm 1 / 2>$ & $\mathbf{0}$ & $80.0 \% \pm \pm 15 / 2>$ \\
\hline 31 & $94.0 \% \mid \pm 3 / 2>$ & 29 & $\begin{array}{l}25.6 \%| \pm 5 / 2>+15.6 \%| \mp 3 / 2>+14.2 \% \\
\pm 3 / 2>\end{array}$ \\
\hline 70 & $\begin{array}{l}40.9 \%| \pm 15 / 2\rangle+28.2 \%| \pm 5 / 2\rangle+10.9 \% \\
| \pm 13 / 2\rangle+10.3 \%| \pm 9 / 2\rangle\end{array}$ & 42 & $\begin{array}{l}20.6 \%| \pm 1 / 2>+16.2 \%| \pm 7 / 2>+13.5 \% \\
\pm 9 / 2>+12.7 \% \mid \mp 1 / 2>\end{array}$ \\
\hline 83 & $55.9 \%| \pm 5 / 2>+22.2 \%| \pm 15 / 2>$ & 101 & $\begin{array}{l}28.2 \%| \pm 13 / 2>+15.1 \%| \pm 11 / 2>+15.3 \% \\
\mp 3 / 2>\end{array}$ \\
\hline 102 & $\begin{array}{l}44.1 \%| \pm 7 / 2>+33.2 \%| \pm 13 / 2>+14.9 \% \\
| \pm 15 / 2\rangle\end{array}$ & 118 & $\begin{array}{l}20.8 \%| \pm 9 / 2>+19.8 \%| \pm 13 / 2>+14.5 \% \\
\pm 11 / 2>+11.0 \% \mid \pm 7 / 2>\end{array}$ \\
\hline 203 & $\begin{array}{l}36.0 \%| \pm 11 / 2>+25.4 \%| \pm 7 / 2>+16.7 \% \\
| \pm 9 / 2\rangle+15.6 \%| \pm 13 / 2\rangle\end{array}$ & 154 & $\begin{array}{l}23.7 \%| \pm 13 / 2>+14.5 \%| \pm 5 / 2>+11.2 \% \\
|\mp 1 / 2>+10.6 \%| \mp 11 / 2\rangle\end{array}$ \\
\hline 241 & $\begin{array}{l}63.8 \%| \pm 9 / 2>+17.2 \%| \pm 11 / 2>+11.6 \% \\
\mid \pm 15 / 2>\end{array}$ & 205 & $\begin{array}{l}19.2 \%| \pm 11 / 2>+16.1 \%| \pm 5 / 2\rangle+12.1 \% \\
\pm 9 / 2>+10.6 \%| \pm 13 / 2\rangle\end{array}$ \\
\hline 247 & $\begin{array}{l}34.9 \%| \pm 13 / 2>+31.0 \%| \pm 11 / 2>+ \\
19.7 \% \mid \pm 7 / 2>\end{array}$ & 290 & $\begin{array}{l}20.0 \%| \pm 9 / 2>+18.1 \%| \pm 11 / 2\rangle+13.0 \% \\
| \pm 7 / 2\rangle+10.5 \%| \pm 3 / 2>+10.1 \%| \pm 1 / 2\rangle\end{array}$ \\
\hline
\end{tabular}

Table S11. Ground multiplet energy level scheme (Kramers doublets in $\mathrm{cm}^{-1}$ ) and main $\left|\mathrm{M}_{\mathrm{J}}\right\rangle$ contributions (> $10 \%)$ to the wave function calculated for MUV-5a(Ho1) and MUV-5a(Ho2).

\begin{tabular}{|c|c|c|c|}
\hline \multicolumn{2}{|r|}{ MUV-5a(Ho1) } & \multicolumn{2}{|r|}{ MUV-5a(Ho2) } \\
\hline $\mathbf{0 . 0}$ & $\begin{array}{l}29.3 \%|+8>+29.3 \%|-8>+12.4 \% \mid+6> \\
+12.4 \% \mid-6>\end{array}$ & $\mathbf{0 . 0}$ & $71.6 \% \mid 0>$ \\
\hline 2.4 & $\begin{array}{l}32.5 \%|+8>+32.5 \%|-8>+13.9 \% \mid+6> \\
+13.9 \% \mid-6>\end{array}$ & 1.2 & $32.4 \%|+1>+32.4 \%|-1>$ \\
\hline 16 & $\begin{array}{l}25.5 \%|+3>+25.5 \%|-3>+19.8 \% \mid+1> \\
+19.8 \% \mid-1>\end{array}$ & 6 & $\begin{array}{l}22.4 \%|+1>+22.4 \%|-1>+10.5 \% \mid+2>+ \\
10.5 \% \mid-2>\end{array}$ \\
\hline 19 & $43.9 \%|+2>+43.9 \%|-2>$ & 10 & $\begin{array}{l}24.5 \%|+2>+24.5 \%|-2>+11.4 \% \mid+1>+ \\
11.4 \% \mid-1>\end{array}$ \\
\hline 37 & $\begin{array}{l}23.6 \%|+1>+23.6 \%|-1>+14.5 \% \mid+3> \\
+14.5 \% \mid-3>\end{array}$ & 48 & $37.6 \%|+7>+37.6 \%|-7\rangle$ \\
\hline 48 & $54.6 \%|0>+15.1 \%|+4>+15.1 \% \mid-4>$ & 66 & $\begin{array}{l}23.1 \%|+7>+23.1 \%|-7>+17.1 \% \mid+8>+ \\
17.1 \% \mid-8>\end{array}$ \\
\hline 61 & $\begin{array}{l}27.6 \%|+7>+27.6 \%|-7>+18.7 \% \mid+5> \\
+18.7 \% \mid-5>\end{array}$ & 82 & $35.1 \%|+8>+35.1 \%|-8\rangle$ \\
\hline 68 & $31.2 \%|+7>+31.2 \%|-7\rangle$ & 87 & $\begin{array}{l}28.8 \%|+8>+28.8 \%|-8>+13.7 \% \mid+7>+ \\
13.7 \% \mid-7>\end{array}$ \\
\hline 128 & $33.3 \%|+4>+33.3 \%|-4>$ & 109 & $26.4 \%|+6>+26.4 \%|-6>$ \\
\hline 136 & $\begin{array}{l}18.1 \%|+5>+18.1 \%|-5>+12.1 \% \mid+1> \\
+12.1 \% \mid-1>\end{array}$ & 123 & $37.1 \%|+6>+37.1 \%|-6>$ \\
\hline 151 & $\begin{array}{l}15.1 \%|+6>+15.1 \%|-6>+12.0 \%|+8\rangle \\
+12.0 \%|-8>+10.2 \%|+2\rangle+10.2 \% \mid- \\
2>\end{array}$ & 148 & $\begin{array}{l}19.3 \%|+5>+19.3 \%|-5>+11.2 \% \mid+4>+ \\
11.2 \% \mid-4>\end{array}$ \\
\hline
\end{tabular}




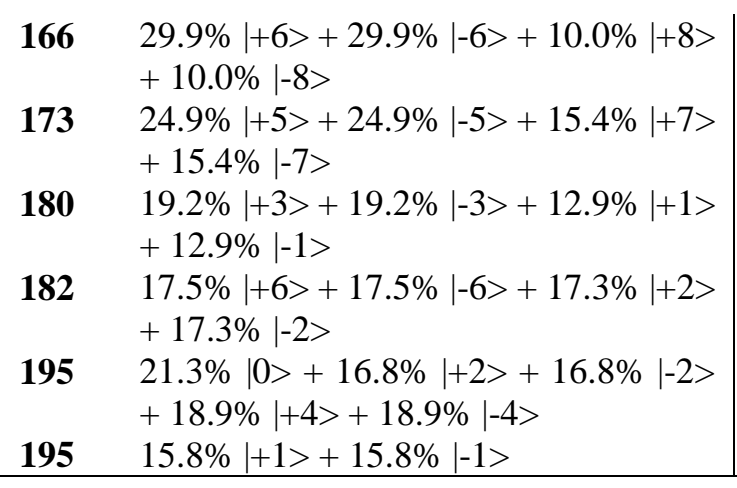

$17022.4 \%|+5>+22.4 \%|-5>+15.7 \% \mid+2>+$ $15.7 \% \mid-2>$

$17529.5 \%|+4>+29.5 \%|-4>$

$17821.6 \%|+3>+21.6 \%|-3>$

$18119.7 \%|+5>+19.7 \%|-5>+10.6 \% \mid+3>+$ $10.6 \% \mid-3>$

$20622.0 \%|+3>+22.0 \%|-3>+14.0 \% \mid+5>+$ $14.0 \% \mid-5>$

$21035.0 \%|+4>+35.0 \%|-4>+12.6 \% \mid 0>$

Table S12. Ground multiplet energy level scheme (Kramers doublets in $\mathrm{cm}^{-1}$ ) and main $\left|\mathrm{M}_{\mathrm{J}}\right\rangle$ contributions (> $10 \%)$ to the wave function calculated for MUV-5a(Er1) and MUV-5a(Er2).

\begin{tabular}{|c|c|c|c|}
\hline \multicolumn{2}{|r|}{ MUV-5a(Er1) } & \multicolumn{2}{|r|}{ MUV-5a(Er2) } \\
\hline $\mathbf{0}$ & $80.1 \%| \pm 13 / 2>+11.7 \%| \pm 15 / 2>$ & $\mathbf{0}$ & $66.6 \%| \pm 15 / 2>+19.0 \%| \pm 9 / 2>$ \\
\hline 11 & $55.7 \%| \pm 11 / 2>+20.4 \%| \pm 9 / 2>$ & 25 & $\begin{array}{l}26.2 \%| \pm 1 / 2>+14.9 \%| \pm 3 / 2>+11.3 \% \\
\mid \pm 7 / 2>\end{array}$ \\
\hline 27 & $\begin{array}{l}38.9 \%| \pm 9 / 2>+14.8 \%| \pm 7 / 2>+12.5 \% \\
| \pm 11 / 2>+10.3 \%| \pm 3 / 2>\end{array}$ & 38 & $41.9 \%| \pm 13 / 2>+13.3 \%| \pm 7 / 2>$ \\
\hline 50 & $\begin{array}{l}29.1 \%| \pm 1 / 2\rangle+21.8 \%| \pm 7 / 2\rangle+11.1 \% \\
| \pm 11 / 2\rangle\end{array}$ & 81 & $\begin{array}{l}29.1 \%| \pm 11 / 2>+15.7 \%| \mp 3 / 2>+11.9 \% \\
| \pm 13 / 2>+10.3 \%| \pm 7 / 2>\end{array}$ \\
\hline 120 & $\begin{array}{l}38.7 \%| \pm 5 / 2>+22.3 \%| \mp 7 / 2>+11.9 \% \\
\mid \pm 3 / 2>\end{array}$ & 116 & $\begin{array}{l}25.7 \%| \pm 5 / 2>+25.2 \%| \pm 3 / 2>+14.5 \% \\
| \pm 9 / 2>+13.2 \%| \pm 11 / 2>\end{array}$ \\
\hline 139 & $37.1 \%| \pm 15 / 2>+33.1 \%| \pm 3 / 2>$ & 142 & $\begin{array}{l}33.6 \%| \pm 1 / 2>+22.1 \%| \pm 5 / 2>+12.1 \% \\
\mid \mp 3 / 2>\end{array}$ \\
\hline 171 & $\begin{array}{l}42.1 \%| \pm 15 / 2>+19.6 \%| \pm 9 / 2>+10.9 \% \\
| \pm 3 / 2>+10.5 \%| \pm 5 / 2>\end{array}$ & 192 & $32.5 \%| \pm 7 / 2>+29.1 \%| \pm 11 / 2>$ \\
\hline 187 & $\begin{array}{l}29.8 \%| \pm 1 / 2>+14.6 \%| \mp 1 / 2\rangle+13.0 \% \\
| \pm 7 / 2>+10.7 \%| \mp 3 / 2\rangle\end{array}$ & 199 & $\begin{array}{l}42.7 \%| \pm 9 / 2>+22.0 \%| \pm 13 / 2>+10.6 \% \\
\mid \pm 7 / 2>\end{array}$ \\
\hline
\end{tabular}




\section{S9. References}

[1] Nowell H, Barnett SA, Christensen KE, Teat SJ, Allan DR. J Synchrotron Radiat., 2012, 19, 435-441.

[2] (a) G. M. Sheldrick, SADABS, empirical absorption correction program based upon the method of Blessing.

(b) L. Krause, R. Herbst-Irmer, G. M. Sheldrick, D. Stalke, An empirical correction for absorption anisotropy J. Appl. Cryst. 2015, 48. (c) R. H. Blessing, An empirical correction for absorption anisotropy, Acta Crystallogr. 1995, A51, 33-38.

[3] a) Sheldrick. G. M. Crystal structure refinement with SHELXL, Acta Crystallogr., 2015, C71, 3-8;

[4] O. V. Dolomanov, L. J. Bourhis, R. J. Gildea, J. A. K. Howard, H. Puschmann, OLEX2: a complete structure solution, refinement and analysis program. J. Appl. Cryst., 2009, 42, 339-341.

[5] T. C. Umland, S. Allie, T. Kuhlmann, P. Coppens, J. Phys. Chem. 1988, 92, 6456-6460.

[6] L. S. Xie, M. Dinca, Isr. J. Chem. 2018, 2139, 1119-1122.

[7] J. Su, T. Hu, R. Murase, H. Wang, D. M. D. Alessandro, M. Kurmoo, J. Zuo, Inorg. Chem. 2019, 58, 36983706.

[8] J.J. Baldoví, J.J. Borrás-Almenar, J.M. Clemente-Juan, E. Coronado, A. Gaita-Ariño, Dalton Trans. 2012, 41, 13705.

[9] J.J. Baldoví, S. Cardona-Serra, J.M. Clemente-Juan, E. Coronado, A. Gaita-Ariño, A. Palii, J. Comput. Chem., 2013, 34, 1961.

[10] (a) C. Rudowicz, C.Y. Chung, J. Phys. Condens. Matter, 2004, 16, 5825; (b) C. Rudowicz, J. Phys. C: Solid State Phys., 1985, 18, 1415; (c) C. Rudowicz, J. Phys. C: Solid State Phys., 1985, 18, 3837 (erratum). [11] I.D. Ryabov, Journal of Magnetic Resonance, 1999, 140, 141.

[12] K. W. H. Stevens, Proc.Phys. Soc. 1952, 65, 209.

[13] S. Edvardsson, M. Klinterberg, Journal of Alloys and Compounds, 1998, 275, 233.

[14] J. Castells-Gil, J. J. Baldoví, Carlos Martí-Gastaldo, G. Mínguez Espallargas, Dalt. Trans. 2018, 47, 1473414740. 
